\title{
Hodge cycles on abelian varieties
}

\author{
P. Deligne (notes by J.S. Milne)
}

July 4, 2003; June 1, 2011; October 1, 2018.

\begin{abstract}
This is a TeXed copy of the article published in

Deligne, P.; Milne, J.S.; Ogus, A.; Shih, Kuang-yen. Hodge cycles, motives, and Shimura varieties. LNM 900, Springer, 1982, pp. 9-100,

somewhat revised and updated. See the endnotes for more details. The original article consisted of the notes (written by jsm) of the seminar "Périodes des Intégrals Abéliennes" given by P. Deligne at I.H.E.S., 1978-79. This file is available at www . jmilne.org/math/Documents.
\end{abstract}

\section{Contents}

Introduction . . . . . . . . . . . . . . . . . . . . . . . . 2

1 Review of cohomology . . . . . . . . . . . . . . . . . 5

Topological manifolds . . . . . . . . . . . . . . . . . . . . 5

Differentiable manifolds . . . . . . . . . . . . . . . . . . . 5

Complex manifolds . . . . . . . . . . . . . . . . . . . . . 7

Complete smooth varieties . . . . . . . . . . . . . . . . . . 7

Applications to periods . . . . . . . . . . . . . . . . . . . . 11

2 Absolute Hodge cycles; principle B . . . . . . . . . . . . . . . 15

Definitions ( $k$ algebraically closed of finite transcendence degree) $. . . \quad . \quad 15$

Basic properties of absolute Hodge cycles . . . . . . . . . . . . . 16

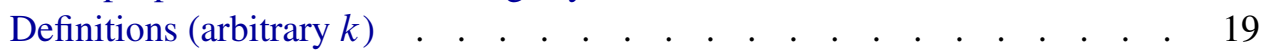

Statement of the main theorem . . . . . . . . . . . . . . . . 19

Principle B . . . . . . . . . . . . . . . . . . . . . . . . 19

3 Mumford-Tate groups; principle A . . . . . . . . . . . . . . . 22

Characterizing subgroups by their fixed tensors . . . . . . . . . . . 22

Hodge structures . . . . . . . . . . . . . . . . . . . . . 23

Mumford-Tate groups . . . . . . . . . . . . . . . . . . . . 24

Principle A . . . . . . . . . . . . . . . . . . . . . . . . . 26

4 Construction of some absolute Hodge cycles . . . . . . . . . . . 28

Hermitian forms . . . . . . . . . . . . . . . . . . . . . . 28

Conditions for $\bigwedge_{E}^{d} H^{1}(A, \mathbb{Q})$ to consist of absolute Hodge cycles . . . . 30

5 Completion of the proof for abelian varieties of CM-type . . . . . . 36

Abelian varieties of CM-type. . . . . . . . . . . . . . . . . . 36

Proof of the main theorem for abelian varieties of CM-type . . . . . . . 37 
6 Completion of the proof; consequences . . . . . . . . . . . . . 41

Completion of the proof of Theorem 2.11 . . . . . . . . . . . . . . . . . 41

Consequences of Theorem 2.11 . . . . . . . . . . . . . . . . . . . . . . . 42

7 Algebraicity of values of the $\Gamma$-function . . . . . . . . . . . . . . 44

The Fermat hypersurface . . . . . . . . . . . . . . . . . . . 45

Calculation of the cohomology . . . . . . . . . . . . . . . . . . 46

The action of $\operatorname{Gal}(\overline{\mathbb{Q}} / k)$ on the étale cohomology . . . . . . . . . . . . . 49

Calculation of the periods . . . . . . . . . . . . . . . . . . . 51

The theorem. . . . . . . . . . . . . . . . . 53

Restatement of the theorem . . . . . . . . . . . . . 55

References. . . . . . . . . . . . . . . . . . . . 57

M Endnotes (by J.S. Milne) . . . . . . . . . . . . . . . . . . . 60

\section{Introduction}

Let $X$ be a smooth projective variety over $\mathbb{C}$. Hodge conjectured that certain cohomology classes on $X$ are algebraic. The main result proved in these notes shows that, when $X$ is an abelian variety, the classes considered by Hodge have many of the properties of algebraic classes.

In more detail, let $X^{\text {an }}$ be the complex analytic manifold associated with $X$, and consider the singular cohomology groups $H^{n}\left(X^{\text {an }}, \mathbb{Q}\right)$. The variety $X^{\text {an }}$ being of Kähler type (every projective embedding defines a Kähler structure), its cohomology groups $H^{n}\left(X^{\text {an }}, \mathbb{C}\right) \simeq$ $H^{n}\left(X^{\text {an }}, \mathbb{Q}\right) \otimes \mathbb{C}$ have canonical decompositions

$$
H^{n}\left(X^{\mathrm{an}}, \mathbb{C}\right)=\bigoplus_{p+q=n} H^{p, q}, \quad H^{p, q} \stackrel{\text { def }}{=} H^{q}\left(X^{\mathrm{an}}, \Omega_{X^{\text {an }}}^{p}\right) .
$$

The cohomology class $c l(Z) \in H^{2 p}\left(X^{\text {an }}, \mathbb{C}\right)$ of an algebraic subvariety $Z$ of codimension $p$ in $X$ is rational (i.e., it lies in $\left.H^{2 p}\left(X^{\text {an }}, \mathbb{Q}\right)\right)$ and is of bidegree $(p, p)$ (i.e., it lies in $H^{p, p}$ ). The Hodge conjecture states that, conversely, every element of

$$
H^{2 p}\left(X^{\text {an }}, \mathbb{Q}\right) \cap H^{p, p}
$$

is a $\mathbb{Q}$-linear combination of the classes of algebraic subvarieties. Since the conjecture is unproven, it is convenient to call these rational $(p, p)$-classes Hodge cycles on $X$.

Now consider a smooth projective variety $X$ over a field $k$ that is of characteristic zero, algebraically closed, and small enough to be embeddable in $\mathbb{C}$. The algebraic de Rham cohomology groups $H_{\mathrm{dR}}^{n}(X / k)$ have the property that, for any embedding $\sigma: k \hookrightarrow \mathbb{C}$, there are canonical isomorphisms

$$
H_{\mathrm{dR}}^{n}(X / k) \otimes_{k, \sigma} \mathbb{C} \simeq H_{\mathrm{dR}}^{n}\left(X^{\mathrm{an}}, \mathbb{C}\right) \simeq H^{n}\left(X^{\mathrm{an}}, \mathbb{C}\right) .
$$

It is natural to say that $t \in H_{\mathrm{dR}}^{2 p}(X / k)$ is a Hodge cycle on $X$ relative to $\sigma$ if its image in $H^{2 p}\left(X^{\text {an }}, \mathbb{C}\right)$ is $(2 \pi i)^{p}$ times a Hodge cycle on $X \otimes_{k, \sigma} \mathbb{C}$. The arguments in these notes show that, if $X$ is an abelian variety, then an element of $H_{\mathrm{dR}}^{2 p}(X / k)$ that is a Hodge cycle on $X$ relative to one embedding of $k$ into $\mathbb{C}$ is a Hodge cycle relative to all embeddings; further, for any embedding, $(2 \pi i)^{p}$ times a Hodge cycle in $H^{2 p}\left(X^{\text {an }}, \mathbb{C}\right)$ always lies in the image 
of $H_{\mathrm{dR}}^{2 p}(X / k) .{ }^{1}$ Thus the notion of a Hodge cycle on an abelian variety is intrinsic to the variety: it is a purely algebraic notion. In the case that $k=\mathbb{C}$ the theorem shows that the image of a Hodge cycle under an automorphism of $\mathbb{C}$ is again a Hodge cycle; equivalently, the notion of a Hodge cycle on an abelian variety over $\mathbb{C}$ does not depend on the map $X \rightarrow$ Spec $\mathbb{C}$. Of course, all this would be obvious if only one knew the Hodge conjecture.

In fact, a stronger result is proved in which a Hodge cycle is defined to be an element of $H_{\mathrm{dR}}^{n}(X) \times \prod_{l} H^{n}\left(X_{\mathrm{et}}, \mathbb{Q}_{l}\right)$. As the title of the original seminar suggests, the stronger result has consequences for the algebraicity of the periods of abelian integrals: briefly, it allows one to prove all arithmetic properties of abelian periods that would follow from knowing the Hodge conjecture for abelian varieties.

In more detail, the main theorem proved in these notes is that every Hodge cycle on an abelian variety (in characteristic zero) is an absolute Hodge cycle — see $§ 2$ for the definitions and Theorem 2.11 for a precise statement of the result.

The proof is based on the following two principles.

A. Let $t_{1}, \ldots, t_{N}$ be absolute Hodge cycles on a smooth projective variety $X$ and let $G$ be the largest algebraic subgroup of $\operatorname{GL}\left(H^{*}(X, \mathbb{Q})\right) \times \mathrm{GL}(\mathbb{Q}(1))$ fixing the $t_{i}$; then every cohomology class $t$ on $X$ fixed by $G$ is an absolute Hodge cycle (see 3.8).

B. If $\left(X_{S}\right)_{s \in S}$ is an algebraic family of smooth projective varieties with $S$ connected and smooth and $\left(t_{S}\right)_{s \in S}$ is a family of rational cycles (i.e., a global section of ...) such that $t_{s}$ is an absolute Hodge cycle for one $s$, then $t_{s}$ is an absolute Hodge cycle for all $s$ (see 2.12, 2.15).

Every abelian variety $A$ with a Hodge cycle $t$ is contained in a smooth algebraic family in which $t$ remains Hodge and which contains an abelian variety of CM-type. Therefore, Principle $\mathrm{B}$ shows that it suffices to prove the main theorem for $A$ an abelian variety of CM-type (see $\S 6$ ). Fix a CM-field $E$, which we can assume to be Galois over $\mathbb{Q}$, and let $\Sigma$ be a set of representatives for the $E$-isogeny classes over $\mathbb{C}$ of abelian varieties with complex multiplication by $E$. Principle B is used to construct some absolute Hodge classes on $\bigoplus_{A \in \Sigma} A-$ the principle allows us to replace $\oplus A$ by an abelian variety of the form $A_{0} \otimes_{\mathbb{Z}} \mathcal{O}_{E}$ (see $\left.\S 4\right)$. Let $G \subset \mathrm{GL}\left(\oplus_{A \in \Sigma} H_{1}(A, \mathbb{Q})\right) \times \mathrm{GL}(\mathbb{Q}(1))$ be the subgroup fixing the absolute Hodge cycles just constructed plus some other (obvious) absolute Hodge cycles. It is shown that $G$ fixes every Hodge cycle on $A$, and Principle A therefore completes the proof (see §5).

On analyzing which properties of absolute Hodge cycles are used in the above proof, one arrives at a slightly stronger result. ${ }^{2}$ Call a rational cohomology class $c$ on a smooth projective complex variety $X$ accessible if it belongs to the smallest family of rational cohomology classes such that:

(a) the cohomology class of every algebraic cycle is accessible;

(b) the pull-back by a map of varieties of an accessible class is accessible;

(c) if $t_{1}, \ldots, t_{N} \in H^{*}(X, \mathbb{Q})$ are accessible, and if a rational class $t$ in some $H^{2 p}(X, \mathbb{Q})$ is fixed by an algebraic subgroup $G$ of $\operatorname{Aut}\left(H^{*}(X, \mathbb{Q})\right.$ ) (automorphisms of $H^{*}(X, \mathbb{Q})$ as a graded algebra) fixing the $t_{i}$, then $t$ is accessible;

\footnotetext{
${ }^{1}$ Added(jsm). This doesn't follow directly from Theorem 2.11 (see 2.4). However, one obtains a variant of Theorem 2.11 using the above definitions simply by dropping the étale component everywhere in the proof (see, for example, 2.10b).

${ }^{2}$ Added(jsm). See also $§ 9$ of Milne, Shimura varieties and moduli, 2013.
} 
(d) Principle $B$ holds with "absolute Hodge" replaced by "accessible".

Sections 4,5,6 of these notes can be interpreted as proving that, when $X$ is an abelian variety, every Hodge cycle is accessible. Sections 2,3 define the notion of an absolute Hodge cycle and show that the family of absolute Hodge cycles satisfies (a), (b), (c), and (d); therefore, an accessible class is absolutely Hodge. We have the implications:

Hodge $\stackrel{\text { abelian varieties }}{=}$ accessible $\Longrightarrow$ absolutely Hodge $\stackrel{\text { trivial }}{\Longrightarrow}$ Hodge.

Only the first implication is restricted to abelian varieties.

The remaining three sections, $\S 1$ and $\S 7$, serve respectively to review the different cohomology theories and to give some applications of the main results to the algebraicity of products of special values of the $\Gamma$-function.

\section{NOTATION}

We define $\mathbb{C}$ to be the algebraic closure of $\mathbb{R}$ and $i \in \mathbb{C}$ to be a square root of -1 ; thus $i$ is only defined up to sign. A choice of $i=\sqrt{-1}$ determines an orientation of $\mathbb{C}$ as a real manifold — we take that for which $1 \wedge i>0$ - and hence an orientation of every complex manifold. Complex conjugation on $\mathbb{C}$ is denoted by $\iota$ or by $z \mapsto \bar{z}$.

Recall that the category of abelian varieties up to isogeny is obtained from the category of abelian varieties by taking the same class of objects but replacing $\operatorname{Hom}(A, B)$ with $\operatorname{Hom}(A, B) \otimes \mathbb{Q}$. We shall always regard an abelian variety as an object in the category of abelian varieties up to isogeny: thus $\operatorname{Hom}(A, B)$ is a vector space over $\mathbb{Q}$.

If $\left(V_{\alpha}\right)$ is a family of representations of an algebraic group $G$ over a field $k$ and $t_{\alpha, \beta} \in V_{\alpha}$, then the subgroup of $G$ fixing the $t_{\alpha, \beta}$ is the algebraic subgroup $H$ of $G$ such that, for all $k$-algebras $R$,

$$
H(R)=\left\{g \in G(R) \mid g\left(t_{\alpha, \beta} \otimes 1\right)=t_{\alpha, \beta} \otimes 1, \text { all } \alpha, \beta\right\} .
$$

Linear duals are denoted by $\vee$. If $X$ is a variety over a field $k$ and $\sigma$ is a homomorphism $\sigma: k \hookrightarrow k^{\prime}$, then $\sigma X$ denotes the variety $X \otimes_{k, \sigma} k^{\prime}\left(=X \times_{\operatorname{Spec}(k)} \operatorname{Spec}\left(k^{\prime}\right)\right)$.

By $a \gg b$ we mean that $a$ is sufficiently greater than $b$. 


\section{Review of cohomology}

\section{Topological manifolds}

Let $X$ be a topological manifold and $F$ a sheaf of abelian groups on $X$. We set

$$
H^{n}(X, F)=H^{n}\left(\Gamma\left(X, F^{\bullet}\right)\right)
$$

where $F \rightarrow F^{\bullet}$ is any acyclic resolution of $F$. This defines $H^{n}(X, F)$ uniquely up to a unique isomorphism.

When $F$ is the constant sheaf defined by a field $K$, these groups can be identified with singular cohomology groups as follows. Let $S_{\bullet}(X, K)$ be the complex in which $S_{n}(X, K)$ is the $K$-vector space with basis the singular $n$-simplices in $X$ and the boundary map sends a simplex to the (usual) alternating sum of its faces. Set

$$
S^{\bullet}(X, K)=\operatorname{Hom}\left(S_{\bullet}(X, K), K\right)
$$

with the boundary map for which

$$
(\alpha, \sigma) \mapsto \alpha(\sigma): S^{\bullet}(X, K) \otimes S_{\bullet}(X, K) \rightarrow K
$$

is a morphism of complexes, namely, that defined by

$$
(d \alpha)(\sigma)=(-1)^{\operatorname{deg}(\alpha)+1} \alpha(d \sigma) .
$$

Proposition 1.1. There is a canonical isomorphism $H^{n}\left(S^{\bullet}(X, K)\right) \rightarrow H^{n}(X, K)$.

Proof. If $U$ is the unit ball, then $H^{0}\left(S^{\bullet}(U, K)\right)=K$ and $H^{n}\left(S^{\bullet}(U, K)\right)=0$ for $n>0$. Thus, $K \rightarrow S^{\bullet}(U, K)$ is a resolution of the group $K$. Let $\mathcal{S}^{n}$ be the sheaf of $X$ associated with the presheaf $V \mapsto S^{n}(V, K)$. The last remark shows that $K \rightarrow \mathcal{S}^{\bullet}$ is a resolution of the sheaf $K$. As each $\mathcal{S}^{n}$ is fine (Warner 1971, 5.32), $H^{n}(X, K) \simeq H^{n}\left(\Gamma\left(X, \mathcal{S}^{\bullet}\right)\right.$ ). But the obvious map $S^{\bullet}(X, K) \rightarrow \Gamma\left(X, \mathcal{S}^{\bullet}\right)$ is surjective with an exact complex as kernel (loc. cit.), and so

$$
H^{n}\left(S^{\bullet}(X, K)\right) \stackrel{\simeq}{\rightarrow} H^{n}\left(\Gamma\left(X, \mathcal{S}^{\bullet}\right)\right) \simeq H^{n}(X, K) .
$$

\section{Differentiable manifolds}

Now assume $X$ is a differentiable manifold. On replacing "singular $n$-simplex" by "differentiable singular $n$-simplex" in the above definitions, one obtains complexes $S_{\bullet}^{\infty}(X, K)$ and $S_{\infty}^{\bullet}(X, K)$. The same argument shows that there is a canonical isomorphism

$$
H_{\infty}^{n}(X, K) \stackrel{\text { def }}{=} H^{n}\left(S_{\bullet}^{\infty}(X, K)\right) \stackrel{\simeq}{\rightarrow} H^{n}(X, K)
$$

(Warner 1971, 5.32).

Let $\mathcal{O}_{X} \infty$ be the sheaf of $C^{\infty}$ real-valued functions on $X$, let $\Omega_{X}^{n}$ be the $\mathcal{O}_{X^{\infty}}$-module of $C^{\infty}$ differential $n$-forms on $X$, and let $\Omega_{X^{\infty}}^{\bullet}$ be the complex

$$
\mathcal{O}_{X^{\infty}} \stackrel{d}{\rightarrow} \Omega_{X^{\infty}}^{1} \stackrel{d}{\rightarrow} \Omega_{X^{\infty}}^{2} \stackrel{d}{\rightarrow} \cdots
$$


The de Rham cohomology groups of $X$ are defined to be

$$
H_{\mathrm{dR}}^{n}(X)=H^{n}\left(\Gamma\left(X, \Omega_{X^{\infty}}^{\bullet}\right)\right)=\frac{\{\text { closed } n \text {-forms }\}}{\{\text { exact } n \text {-forms }\}} .
$$

If $U$ is the unit ball, Poincaré's lemma shows that $H_{\mathrm{dR}}^{0}(U)=\mathbb{R}$ and $H_{\mathrm{dR}}^{n}(U)=0$ for $n>0$. Thus, $\mathbb{R} \rightarrow \Omega_{X^{\infty}}^{\bullet}$ is a resolution of the constant sheaf $\mathbb{R}$, and as the sheaves $\Omega_{X^{\infty}}^{n}$ are fine (Warner 1971, 5.28), we have $H^{n}(X, \mathbb{R}) \simeq H_{\mathrm{dR}}^{n}(X)$.

For $\omega \in \Gamma\left(X, \Omega_{X^{\infty}}^{n}\right)$ and $\sigma \in S_{n}^{\infty}(X, \mathbb{R})$, define

$$
\langle\omega, \sigma\rangle=(-1)^{\frac{n(n+1)}{2}} \int_{\sigma} \omega \in \mathbb{R} .
$$

Stokes's theorem states that $\int_{\sigma} d \omega=\int_{d \sigma} \omega$, and so

$$
\langle d \omega, \sigma\rangle+(-1)^{n}\langle\omega, d \sigma\rangle=0 .
$$

The pairing $\langle$,$\rangle therefore defines a map of complexes$

$$
f: \Gamma\left(X, \Omega_{X^{\infty}}^{\bullet}\right) \rightarrow S_{\infty}^{\bullet}(X, \mathbb{R}) .
$$

TheOREM 1.2 (DE RHAM). The map $H_{d R}^{n}(X) \rightarrow H_{\infty}^{n}(X, \mathbb{R})$ defined by $f$ is an isomorphism for all $n$.

ProOF. The map is inverse to the map

$$
H_{\infty}^{n}(X, \mathbb{R}) \stackrel{\simeq}{\rightarrow} H^{n}(X, \mathbb{R}) \simeq H_{\mathrm{dR}}^{n}(X)
$$

defined in the previous two paragraphs (Warner 1971, 5.36). (Our signs differ from the usual signs because the standard sign conventions

$$
\int_{\sigma} d \omega=\int_{d \sigma} \omega, \quad \int_{X \times Y} p r_{1}^{*} \omega \wedge p r_{2}^{*} \eta=\int_{X} \omega \cdot \int_{Y} \eta, \quad \text { etc. }
$$

violate the sign conventions for complexes.)

A number $\int_{\sigma} \omega, \sigma \in H_{n}(X, \mathbb{Q})$, is called a period of $\omega$. The map in (1.2) identifies $H^{n}(X, \mathbb{Q})$ with the space of classes of closed forms whose periods are all rational. Theorem 1.2 can be restated as follows: a closed differential form is exact if all its periods are zero; there exists a closed differential form having arbitrarily assigned periods on an independent set of cycles.

Remark 1.3 (Singer And Thorpe 1967, 6.2). If $X$ is compact, then it has a smooth triangulation $T$. Define $S_{\bullet}(X, T, K)$ and $S^{\bullet}(X, T, K)$ as before, but using only simplices in $T$. Then the map

$$
\Gamma\left(X, \Omega_{X^{\infty}}^{\bullet}\right) \rightarrow S^{\bullet}(X, T, K)
$$

defined by the same formulas as $f$ above induces isomorphisms

$$
H_{\mathrm{dR}}^{n}(X) \rightarrow H^{n}\left(S^{\bullet}(X, T, K)\right) .
$$




\section{Complex manifolds}

Now let $X$ be a complex manifold, and let $\Omega_{X^{\text {an }}}^{\bullet}$ denote the complex

$$
\mathcal{O}_{X^{\text {an }}} \stackrel{d}{\rightarrow} \Omega_{X}^{1} \stackrel{d}{\text { an }} \Omega_{X}^{2} \stackrel{d}{\text { an }} \rightarrow \cdots
$$

in which $\Omega_{X^{\text {an }}}^{n}$ is the sheaf of holomorphic differential $n$-forms. Thus, locally a section of $\Omega_{X^{\text {an }}}^{n}$ is of the form

$$
\omega=\sum \alpha_{i_{1} \ldots i_{n}} d z_{i_{1}} \wedge \ldots \wedge d z_{i_{n}}
$$

with $\alpha_{i_{1} \ldots i_{n}}$ a holomorphic function and the $z_{i}$ complex local coordinates. The complex form of Poincaré's lemma shows that $\mathbb{C} \rightarrow \Omega_{X^{\text {an }}}^{\bullet}$ is a resolution of the constant sheaf $\mathbb{C}$, and so there is a canonical isomorphism

$$
H^{n}(X, \mathbb{C}) \rightarrow \mathbb{H}^{n}\left(X, \Omega_{X^{\text {an }}}^{\bullet}\right) \quad \text { (hypercohomology). }
$$

If $X$ is a compact Kähler manifold, then the spectral sequence

$$
E_{1}^{p, q}=H^{q}\left(X, \Omega_{X^{\text {an }}}^{p}\right) \Longrightarrow \mathbb{H}^{p+q}\left(X, \Omega_{X^{\text {an }}}\right)
$$

degenerates, and so provides a canonical splitting

$$
H^{n}(X, \mathbb{C})=\bigoplus_{p+q=n} H^{q}\left(X, \Omega_{X^{\text {an }}}^{p}\right) \quad \text { (the Hodge decomposition) }
$$

as $H^{p, q} \stackrel{\text { def }}{=} H^{q}\left(X, \Omega_{X}^{p}\right)$ is the complex conjugate of $H^{q, p}$ relative to the real structure $H^{n}(X, \mathbb{R}) \otimes \mathbb{C} \simeq H^{n}(X, \mathbb{C})$ (Weil 1958). The decomposition has the following explicit description: the complex $\Omega_{X}^{\bullet} \otimes \mathbb{C}$ of sheaves of complex-valued differential forms on the underlying differentiable manifold is an acyclic resolution of $\mathbb{C}$, and so $H^{n}(X, \mathbb{C})=$ $H^{n}\left(\Gamma\left(X, \Omega_{X^{\infty}}^{\bullet} \otimes \mathbb{C}\right)\right)$; Hodge theory shows that each element of the second group is represented by a unique harmonic $n$-form, and the decomposition corresponds to the decomposition of harmonic $n$-forms into sums of harmonic $(p, q)$-forms, $p+q=n .^{3}$

\section{Complete smooth varieties}

Finally, let $X$ be a complete smooth variety over a field $k$ of characteristic zero. If $k=\mathbb{C}$, then $X$ defines a compact complex manifold $X^{\text {an }}$, and there are therefore groups $H^{n}\left(X^{\text {an }}, \mathbb{Q}\right)$, depending on the map $X \rightarrow \operatorname{Spec}(\mathbb{C})$, that we shall write $H_{\mathrm{B}}^{n}(X)$ (here $B$ abbreviates Betti). If $X$ is projective, then the choice of a projective embedding determines a Kähler structure on $X^{\text {an }}$, and hence a Hodge decomposition (which is independent of the choice of the embedding because it is determined by the Hodge filtration, and the Hodge filtration depends only on $X$; see Theorem 1.4 below). In the general case, we refer to Deligne 1968, 5.3, 5.5, for the existence of the decomposition.

For an arbitrary field $k$ and an embedding $\sigma: k \hookrightarrow \mathbb{C}$, we write $H_{\sigma}^{n}(X)$ for $H_{\mathrm{B}}^{n}(\sigma X)$ and $H_{\sigma}^{p, q}(X)$ for $H^{p, q}(\sigma X)$. As $\iota$ defines a homeomorphism $\sigma X^{\text {an }} \rightarrow \iota \sigma X^{\text {an }}$, it induces an isomorphism $H_{l \sigma}^{n}(X) \rightarrow H_{\sigma}^{n}(X)$. Sometimes, when $k$ is given as a subfield of $\mathbb{C}$, we write $H_{\mathrm{B}}^{n}(X)$ for $H_{\mathrm{B}}^{n}\left(X_{\mathbb{C}}\right)$.

\footnotetext{
${ }^{3}$ For a recent account of Hodge theory, see C. Voisin, Hodge Theory and Complex Algebraic Geometry, I, CUP, 2002.
} 
Let $\Omega_{X / k}^{\bullet}$ denote the complex in which $\Omega_{X / k}^{n}$ is the sheaf of algebraic differential $n$-forms, and define the (algebraic) de Rham cohomology group $H_{\mathrm{dR}}^{n}(X / k)$ to be $\mathbb{H}^{n}\left(X_{\mathrm{Zar}}, \Omega_{X / k}^{\bullet}\right)$ (hypercohomology with respect to the Zariski cohomology). For any homomorphism $\sigma: k \hookrightarrow k^{\prime}$, there is a canonical isomorphism

$$
H_{\mathrm{dR}}^{n}(X / k) \otimes_{k, \sigma} k^{\prime} \rightarrow H_{\mathrm{dR}}^{n}\left(X \otimes_{k} k^{\prime} / k^{\prime}\right) .
$$

The spectral sequence

$$
E_{1}^{p, q}=H^{q}\left(X_{\mathrm{Zar}}, \Omega_{X / k}^{p}\right) \Longrightarrow \mathbb{H}^{p+q}\left(X_{\mathrm{Zar}}, \Omega_{X / k}^{\bullet}\right)
$$

defines a filtration (the Hodge filtration) $F^{p} H_{\mathrm{dR}}^{n}(X)$ on $H_{\mathrm{dR}}^{n}(X)$ which is stable under base change.

THEOREM 1.4. When $k=\mathbb{C}$ the obvious maps

$$
X^{\text {an }} \rightarrow X_{Z a r}, \quad \Omega_{X^{\text {an }}}^{\bullet} \leftarrow \Omega_{X}^{\bullet},
$$

induce isomorphisms

$$
H_{d R}^{n}(X) \rightarrow H_{d R}^{n}\left(X^{a n}\right) \simeq H^{n}\left(X^{a n}, \mathbb{C}\right)
$$

under which $F^{p} H_{d R}^{n}(X)$ corresponds to $F^{p} H^{n}\left(X^{\text {an }}, \mathbb{C}\right) \stackrel{\text { def }}{=} \bigoplus_{p^{\prime} \geq p, p^{\prime}+q^{\prime}=n} H^{p^{\prime}, q^{\prime}}$.

Proof. The initial terms of the spectral sequences

$$
\begin{gathered}
E_{1}^{p, q}=H^{q}\left(X_{\mathrm{Zar}}, \Omega_{X / k}^{p}\right) \Longrightarrow \mathbb{H}^{p+q}\left(X_{\mathrm{Zar}}, \Omega_{X / k}^{\bullet}\right) \\
E_{1}^{p, q}=H^{q}\left(X, \Omega_{X^{\text {an }}}^{p}\right) \Longrightarrow \mathbb{H}^{p+q}\left(X, \Omega_{X_{\text {an }}}^{\bullet}\right)
\end{gathered}
$$

are isomorphic - see Serre 1956 for the projective case and Grothendieck 1966 for the general case. The theorem follows from this because, by definition of the Hodge decomposition, the filtration of $H_{\mathrm{dR}}^{n}\left(X^{\mathrm{an}}\right)$ defined by the above spectral sequence is equal to the filtration of $H^{n}\left(X^{\text {an }}, \mathbb{C}\right)$ defined in the statement of the theorem.

It follows from the theorem and the discussion preceding it that every embedding $\sigma: k \hookrightarrow \mathbb{C}$ defines an isomorphism

$$
H_{\mathrm{dR}}^{n}(X) \otimes_{k, \sigma} \mathbb{C} \stackrel{\simeq}{\rightarrow} H_{\sigma}^{n}(X) \otimes_{\mathbb{Q}} \mathbb{C}
$$

and, in particular, a $k$-structure on $H_{\sigma}^{n}(X) \otimes_{\mathbb{Q}} \mathbb{C}$. When $k=\mathbb{Q}$, this structure should be distinguished from the $\mathbb{Q}$-structure defined by $H_{\sigma}^{n}(X)$ : the two are related by the periods.

When $k$ is algebraically closed, we write $H^{n}\left(X, \mathbb{A}_{f}\right)$, or $H_{\mathrm{et}}^{n}(X)$, for $H^{n}\left(X_{\mathrm{et}}, \hat{\mathbb{Z}}\right) \otimes_{\mathbb{Z}}$ $\mathbb{Q}$, where $H^{n}\left(X_{\mathrm{et}}, \hat{\mathbb{Z}}\right)=\lim _{m} H^{n}\left(X_{\mathrm{et}}, \mathbb{Z} / m \mathbb{Z}\right)$ (étale cohomology). If $X$ is connected, $H^{0}\left(X, \mathbb{A}_{f}\right)=\mathbb{A}_{f}$, the ring of finite adèles for $\mathbb{Q}$, which justifies the first notation. By definition, $H_{\mathrm{et}}^{n}(X)$ depends only on $X$ (and not on its structure morphism $X \rightarrow$ Spec $k$ ). The map $H_{\mathrm{et}}^{n}(X) \rightarrow H_{\mathrm{et}}^{n}\left(X \otimes_{k} k^{\prime}\right)$ defined by an inclusion $k \hookrightarrow k^{\prime}$ of algebraically closed fields is an isomorphism (special case of the proper base change theorem Artin et al. 1973, XII). The comparison theorem (ibid. XI) shows that, when $k=\mathbb{C}$, there is a canonical isomorphism $H_{\mathrm{B}}^{n}(X) \otimes \mathbb{A}_{f} \rightarrow H_{\mathrm{et}}^{n}(X)$. It follows that $H_{\mathrm{B}}^{n}(X) \otimes \mathbb{A}_{f}$ is independent of the morphism $X \rightarrow \operatorname{Spec} \mathbb{C}$, and that, over any algebraically closed field of characteristic zero, $H_{\mathrm{et}}^{n}(X)$ is a free $\mathbb{A}_{f}$-module. 
The $\mathbb{A}_{f}$-module $H^{n}\left(X, \mathbb{A}_{f}\right)$ can also be described as the restricted product of the spaces $H^{n}\left(X, \mathbb{Q}_{l}\right), l$ a prime number, with respect to the subspaces $H^{n}\left(X, \mathbb{Z}_{l}\right) /\{$ torsion $\}$.

Next we define the notion of the "Tate twist" in each of the three cohomology theories. For this we shall define objects $\mathbb{Q}(1)$ and set $H^{n}(X)(m)=H^{n}(X) \otimes \mathbb{Q}(1)^{\otimes m}$. We want $\mathbb{Q}(1)$ to be $H^{2}\left(\mathbb{P}^{1}\right)$ (realization of the Tate motive in the cohomology theory), but to avoid the possibility of introducing sign ambiguities we shall define it directly,

$$
\begin{aligned}
& \mathbb{Q}_{\mathrm{B}}(1)=2 \pi i \mathbb{Q} \\
& \mathbb{Q}_{\mathrm{et}}(1)=\mathbb{A}_{f}(1) \stackrel{\text { def }}{=}\left(\stackrel{\lim }{r}_{r} \mu_{r}\right) \otimes_{\mathbb{Z}} \mathbb{Q}, \quad \mu_{r}=\left\{\zeta \in k \mid \zeta^{r}=1\right\} \\
& \mathbb{Q}_{\mathrm{dR}}(1)=k,
\end{aligned}
$$

and so

$$
\begin{aligned}
& H_{\mathrm{B}}^{n}(X)(m)=H_{\mathrm{B}}^{n}(X) \otimes_{\mathbb{Q}}(2 \pi i)^{m} \mathbb{Q}=H^{n}\left(X^{\mathrm{an}},(2 \pi i)^{m} \mathbb{Q}\right) \quad(k=\mathbb{C}) \\
& H_{\mathrm{et}}^{n}(X)(m)=H_{\mathrm{et}}^{n}(X) \otimes_{\mathbb{A}_{f}}\left(\mathbb{A}_{f}(1)\right)^{\otimes m}=\left(\lim _{r} H^{n}\left(X_{\mathrm{et}}, \mu_{r}^{\otimes m}\right)\right) \otimes_{\mathbb{Z}} \mathbb{Q} \quad(k \text { alg. closed }) \\
& H_{\mathrm{dR}}^{n}(X)(m)=H_{\mathrm{dR}}^{n}(X) .
\end{aligned}
$$

These definitions extend in an obvious way to negative $m$. For example, we set $\mathbb{Q}_{\mathrm{et}}(-1)=$ $\operatorname{Hom}_{\mathbb{A}_{f}}\left(\mathbb{A}_{f}(1), \mathbb{A}_{f}\right)$ and define

$$
H_{\mathrm{et}}^{n}(X)(-m)=H_{\mathrm{et}}^{n}(X) \otimes \mathbb{Q}_{\mathrm{et}}(-1)^{\otimes m} .
$$

There are canonical isomorphisms

$$
\begin{aligned}
\mathbb{Q}_{\mathrm{B}}(1) \otimes_{\mathbb{Q}} \mathbb{A}_{f} & \rightarrow \mathbb{Q}_{\mathrm{et}}(1) \quad(k \subset \mathbb{C}, k \text { algebraically closed }) \\
\mathbb{Q}_{\mathrm{B}}(1) \otimes \mathbb{C} & \rightarrow \mathbb{Q}_{\mathrm{dR}}(1) \otimes_{k} \mathbb{C} \quad(k \subset \mathbb{C})
\end{aligned}
$$

and hence canonical isomorphisms (the comparison isomorphisms)

$$
\begin{aligned}
H_{\mathrm{B}}^{n}(X)(m) \otimes_{\mathbb{Q}} \mathbb{A}_{f} & \rightarrow H_{\mathrm{et}}^{n}(X)(m) \quad(k \subset \mathbb{C}, k \text { algebraically closed }) \\
H_{\mathrm{B}}^{n}(X)(m) \otimes_{\mathbb{Q}} \mathbb{C} & \rightarrow H_{\mathrm{dR}}^{n}(X)(m) \otimes_{k} \mathbb{C} \quad(k \subset \mathbb{C}) .
\end{aligned}
$$

To define the first, note that exp defines an isomorphism

$$
z \mapsto e^{z}: 2 \pi i \mathbb{Z} / r 2 \pi i \mathbb{Z} \rightarrow \mu_{r} .
$$

After passing to the inverse limit over $r$ and tensoring with $\mathbb{Q}$, we obtain the required isomorphism $2 \pi i \mathbb{A}_{f} \rightarrow \mathbb{A}_{f}(1)$. The second isomorphism is induced by the inclusions

$$
2 \pi i \mathbb{Q} \hookrightarrow \mathbb{C} \hookleftarrow k .
$$

Although the Tate twist for de Rham cohomology is trivial, it should not be ignored. For example, when $k=\mathbb{C}$,

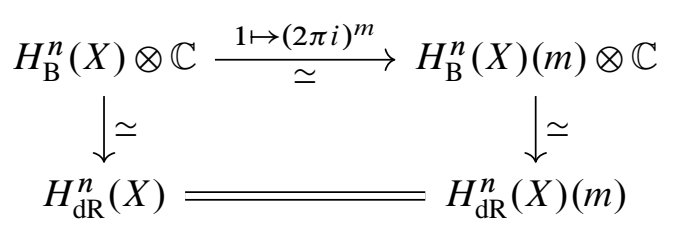


fails to commute by a factor $(2 \pi i)^{m}$. Moreover when $m$ is odd the top isomorphism is defined only up to sign.

In each cohomology theory there is a canonical way of attaching a class $\operatorname{cl}(Z)$ in $H^{2 p}(X)(p)$ to an algebraic cycle $Z$ on $X$ of pure codimension $p$. Since our cohomology groups are without torsion, we can do this using Chern classes (Grothendieck 1958). Starting with a functorial isomorphism $c_{1}: \operatorname{Pic}(X) \rightarrow H^{2}(X)(1)$, one uses the splitting principle to define the Chern polynomial

$$
c_{t}(E)=\sum c_{p}(E) t^{p}, \quad c_{p}(E) \in H^{2 p}(X)(p),
$$

of a vector bundle $E$ on $X$. The map $E \mapsto c_{t}(E)$ is additive, and therefore factors through the Grothendieck group of the category of vector bundles on $X$. But, as $X$ is smooth, this group is the same as the Grothendieck group of the category of coherent $\mathcal{O}_{X}$-modules, and we can therefore define

$$
\operatorname{cl}(Z)=\frac{1}{(p-1) !} c_{p}\left(\mathcal{O}_{Z}\right)
$$

(loc. cit. 4.3).

In defining $c_{1}$ for the Betti and étale theories, we begin with maps

$$
\begin{aligned}
& \operatorname{Pic}(X) \rightarrow H^{2}\left(X^{\text {an }}, 2 \pi i \mathbb{Z}\right) \\
& \operatorname{Pic}(X) \rightarrow H^{2}\left(X_{\text {et }}, \mu_{r}\right)
\end{aligned}
$$

arising as connecting homomorphisms from the sequences

$$
\begin{aligned}
& 0 \rightarrow 2 \pi i \rightarrow \mathcal{O}_{X^{\text {an }}} \stackrel{\text { exp }}{\rightarrow} \mathcal{O}_{X^{\text {an }}}^{\times} \rightarrow 0 \\
& 0 \rightarrow \mu_{r} \rightarrow \mathcal{O}_{X}^{\times} \stackrel{r}{\rightarrow} \mathcal{O}_{X}^{\times} \rightarrow 0 .
\end{aligned}
$$

For the de Rham theory, we note that the $d \log$ map, $f \mapsto \frac{d f}{f}$, defines a map of complexes

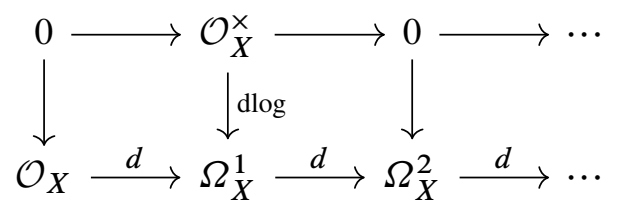

and hence a map

$$
\begin{aligned}
\operatorname{Pic}(X) & \simeq H^{1}\left(X, \mathcal{O}_{X}^{\times}\right) \simeq \mathbb{H}^{2}\left(X, 0 \rightarrow \mathcal{O}_{X}^{\times} \rightarrow \cdots\right) \\
& \rightarrow \mathbb{H}^{2}\left(X, \Omega_{X}^{\bullet}\right)=H_{\mathrm{dR}}^{2}(X)=H_{\mathrm{dR}}^{2}(X)(1)
\end{aligned}
$$

whose negative is $c_{1}$. It can be checked that the three maps $c_{1}$ are compatible with the comparison isomorphisms (Deligne 1971a, 2.2.5.1), and it follows formally that the maps $c l$ are also compatible once one has checked that the Gysin maps and multiplicative structures are compatible with the comparison isomorphisms.

When $k=\mathbb{C}$, there is a direct way of defining a class $\operatorname{cl}(Z) \in H_{2 d-2 p}(X(\mathbb{C}), \mathbb{Q})$ (singular cohomology, $d=\operatorname{dim}(X), p=\operatorname{codim}(Z)$ ): the choice of an $i=\sqrt{-1}$ determines an orientation of $X$ and of the smooth part of $Z$, and there is therefore a topologically defined class $c l(Z) \in H_{2 d-2 p}(X(\mathbb{C}), \mathbb{Q})$. This class has the property that for $[\omega] \in H^{2 d-2 p}\left(X^{\infty}, \mathbb{R}\right)=H^{2 d-2 p}\left(\Gamma\left(X, \Omega_{X^{\infty}}^{\bullet}\right)\right)$ represented by the closed form $\omega$,

$$
\langle\operatorname{cl}(Z),[\omega]\rangle=\int_{Z} \omega .
$$


By Poincaré duality, $c l(Z)$ corresponds to a class $c l_{\text {top }}(Z) \in H_{\mathrm{B}}^{2 p}(X)$, whose image in $H_{\mathrm{B}}^{2 p}(X)(p)$ under the map induced by $1 \mapsto(2 \pi i)^{p}: \mathbb{Q} \rightarrow \mathbb{Q}(p)$ is known to be $c l_{\mathrm{B}}(Z)$. The above formula becomes

$$
\int_{X} c l_{\text {top }}(Z) \cup[w]=\int_{Z} \omega .
$$

There are trace maps $(d=\operatorname{dim} X)$

$$
\begin{aligned}
\operatorname{Tr}_{\mathrm{B}}: H_{\mathrm{B}}^{2 d}(X)(d) \stackrel{\simeq}{\rightarrow} \mathbb{Q} \\
\operatorname{Tr}_{\mathrm{et}}: H_{\mathrm{et}}^{2 d}(X)(d) \stackrel{\simeq}{\rightarrow} \mathbb{A} f \\
\operatorname{Tr}_{\mathrm{dR}}: H_{\mathrm{dR}}^{2 d}(X)(d) \stackrel{\stackrel{\simeq}{\rightarrow}}{ } k
\end{aligned}
$$

that are determined by the requirement that $\operatorname{Tr}(\operatorname{cl}$ (point) $)=1$. They are compatible with the comparison isomorphisms. When $k=\mathbb{C}, \operatorname{Tr}_{\mathrm{B}}$ and $\operatorname{Tr}_{\mathrm{dR}}$ are equal respectively to the composites

$$
\begin{aligned}
& H_{\mathrm{B}}^{2 d}(X)(d) \stackrel{(2 \pi i)^{d} \mapsto 1}{\longrightarrow} H_{\mathrm{B}}^{2 d}(X) \stackrel{\simeq}{\longrightarrow} H^{2 d}\left(\Gamma\left(\Omega_{X^{\infty}}^{\bullet}\right)\right) \stackrel{[\omega] \mapsto \int_{X} \omega}{\longrightarrow} \mathbb{C} \\
& H_{\mathrm{dR}}^{2 d}(X)(d)=H_{\mathrm{dR}}^{2 d}(X) \longrightarrow H^{2 d}\left(\Gamma\left(\Omega_{X^{\infty}}^{\bullet}\right) \stackrel{[\omega] \mapsto \frac{1}{(2 \pi i)^{d}} \int_{X} \omega}{\longrightarrow} \mathbb{C}\right.
\end{aligned}
$$

where we have chosen an $i$ and used it to orientate $X$ (the composite maps are obviously independent of the choice of $i$ ). The formulas of the last paragraph show that

$$
\operatorname{Tr}_{\mathrm{dR}}\left(c l_{\mathrm{dR}}(Z) \cup[\omega]\right)=\frac{1}{(2 \pi i)^{\operatorname{dim} Z}} \int_{Z} \omega .
$$

A definition of $\operatorname{Tr}_{\text {et }}$ can be found in Milne 1980, VI 11.

\section{Applications to periods}

We now deduce some consequences concerning periods.

PROPOSITION 1.5. Let $X$ be a complete smooth variety over an algebraically closed field $k \subset \mathbb{C}$ and let $Z$ be an algebraic cycle on $X_{\mathbb{C}}$ of dimension $r$. For any $C^{\infty}$ differential $r$-form $\omega$ on $X_{\mathbb{C}}$ whose class $[\omega]$ in $H_{d R}^{2 r}\left(X_{\mathbb{C}}\right)$ lies in $H_{d R}^{2 r}(X)$

$$
\int_{Z} \omega \in(2 \pi i)^{r} k
$$

Proof. We first note that $Z$ is algebraically equivalent to a cycle $Z_{0}$ defined over $k$. In proving this, we can assume $Z$ to be prime. There exists a smooth variety $T$ over $k$, a subvariety $\mathcal{Z} \subset X \times T$ that is flat over $T$, and a point $\operatorname{Spec} \mathbb{C} \rightarrow T$ such that $Z=\mathcal{Z} \times_{T} \operatorname{Spec} \mathbb{C}$ in $X \times_{T}$ Spec $\mathbb{C}=X_{\mathbb{C}}$. We can therefore take $Z_{0}$ to be $Z \times_{T}$ Spec $k \subset X \times_{T}$ Spec $k=X$ for any point Spec $k \rightarrow T$. From this it follows that $c l_{\mathrm{dR}}(Z)=c l_{\mathrm{dR}}\left(Z_{0}\right) \in H_{\mathrm{dR}}^{2 r}(X)(r)$ and $\operatorname{Tr}_{\mathrm{dR}}\left(c l_{\mathrm{dR}}(Z) \cup[\omega]\right) \in k$. But we saw above that $\int_{Z} \omega=(2 \pi i)^{r} \operatorname{Tr}_{\mathrm{dR}}\left(c l_{\mathrm{dR}}(Z) \cup[\omega]\right)$.

We next derive a classical relation between the periods of an elliptic curve. For a complete smooth curve $X$ and an open affine subset $U$, the map

$$
H_{\mathrm{dR}}^{1}(X) \rightarrow H_{\mathrm{dR}}^{1}(U)=\frac{\Gamma\left(U, \Omega_{X}^{1}\right)}{d \Gamma\left(U, \mathcal{O}_{X}\right)}=\frac{\{\text { meromorphic diffls, holomorphic on } U\}}{\{\text { exact differentials on } U\}}
$$


is injective with image the set of classes represented by forms whose residues are all zero (such forms are said to be of the second kind). When $k=\mathbb{C}, \operatorname{Tr}_{\mathrm{dR}}([\alpha] \cup[\beta]$ ), where $\alpha$ and $\beta$ are differential 1-forms of the second kind, can be computed as follows. Let $\Sigma$ be the finite set of points where $\alpha$ or $\beta$ has a pole. For $z$ a local parameter at $P \in \Sigma, \alpha$ can be written

$$
\alpha=\sum_{-\infty \ll i<\infty} a_{i} z^{i} d z \text { with } a_{-1}=0 .
$$

There therefore exists a meromorphic function $f$ defined near $P$ such that $d f=\alpha$. We write $\int \alpha$ for any such function - it is defined up to a constant. As $\operatorname{Res}_{P} \beta=0, \operatorname{Res} P\left(\int \alpha\right) \beta$ is well-defined, and one proves that

$$
\operatorname{Tr}_{\mathrm{dR}}([\alpha] \cup[\beta])=\sum_{P \in \Sigma} \operatorname{Res}_{P}\left(\int \alpha\right) \beta .
$$

Now let $X$ be the elliptic curve

$$
y^{2} z=4 x^{3}-g_{2} x z^{2}-g_{3} z^{3} .
$$

There is a lattice $\Lambda$ in $\mathbb{C}$ and corresponding Weierstrass function $\wp(z)$ such that

$$
z \mapsto\left(\wp(z): \wp^{\prime}(z): 1\right)
$$

defines an isomorphism $\mathbb{C} / \Lambda \rightarrow X(\mathbb{C})$. Let $\gamma_{1}$ and $\gamma_{2}$ be generators of $\Lambda$ such that the bases $\left\{\gamma_{1}, \gamma_{2}\right\}$ and $\{1, i\}$ of $\mathbb{C}$ have the same orientation. We can regard $\gamma_{1}$ and $\gamma_{2}$ as elements of $H_{1}(X, \mathbb{Z})$, and then $\gamma_{1} \cdot \gamma_{2}=1$. The differentials $\omega=d x / y$ and $\eta=x d x / y$ on $X$ pull back to $d z$ and $\wp(z) d z$ respectively on $\mathbb{C}$. The first is therefore holomorphic and the second has a single pole at $\infty=(0: 1: 0)$ on $X$ with residue zero (because $0 \in \mathbb{C}$ maps to $\infty \in X$ and $\left.\wp(z)=\frac{1}{z^{2}}+a_{2} z^{2}+\ldots\right)$. We find that

$$
\operatorname{Tr}_{\mathrm{dR}}([\omega] \cup[\eta])=\operatorname{Res}_{0}\left(\int d z\right) \wp(z) d z=\operatorname{Res}_{0}(z \wp(z) d z)=1 .
$$

For $i=1,2$, let

$$
\begin{gathered}
\int_{\gamma_{i}} \frac{d x}{y} \stackrel{\text { def }}{=} \int_{\gamma_{i}} \frac{d x}{\sqrt{4 x^{3}-g_{2} x-g_{3}}}=\omega_{i} \\
\int_{\gamma_{i}} \frac{x d x}{y} \stackrel{\text { def }}{=} \int_{\gamma_{i}} \frac{x d x}{\sqrt{4 x^{3}-g_{2} x-g_{3}}}=\eta_{i}
\end{gathered}
$$

be the periods of $\omega$ and $\eta$. Under the map

$$
H_{\mathrm{dR}}^{1}(X) \rightarrow H^{1}(X, \mathbb{C})
$$

$\omega$ maps to $\omega_{1} \gamma_{1}^{\prime}+\omega_{2} \gamma_{2}^{\prime}$ and $\eta$ maps to $\eta_{1} \gamma_{1}^{\prime}+\eta_{2} \gamma_{2}^{\prime}$, where $\left\{\gamma_{1}^{\prime}, \gamma_{2}^{\prime}\right\}$ is the basis dual to $\left\{\gamma_{1}, \gamma_{2}\right\}$. Thus

$$
\begin{aligned}
1 & =\operatorname{Tr}_{\mathrm{dR}}([\omega] \cup[\eta]) \\
& =\operatorname{Tr}_{\mathrm{B}}\left(\left(\omega_{1} \gamma_{1}^{\prime}+\omega_{2} \gamma_{2}^{\prime}\right) \cup\left(\eta_{1} \gamma_{1}^{\prime}+\eta_{2} \gamma_{2}^{\prime}\right)\right) \\
& =\left(\omega_{1} \eta_{2}-\omega_{2} \eta_{1}\right) \operatorname{Tr}_{\mathrm{B}}\left(\gamma_{1}^{\prime} \cup \gamma_{2}^{\prime}\right) \\
& =\frac{1}{2 \pi i}\left(\omega_{1} \eta_{2}-\omega_{2} \eta_{1}\right) .
\end{aligned}
$$


Hence

$$
\omega_{1} \eta_{2}-\omega_{2} \eta_{1}=2 \pi i
$$

This is the Legendre relation.

The next proposition shows how the existence of algebraic cycles can force algebraic relations between the periods of abelian integrals. Let $X$ be an abelian variety over a subfield $k$ of $\mathbb{C}$. In each of the three cohomology theories,

$$
H^{r}(X)=\bigwedge^{r} H^{1}(X)
$$

and

$$
H^{1}(X \times X \times \cdots)=H^{1}(X) \oplus H^{1}(X) \oplus \cdots
$$

Let $v \in \mathbb{G}_{m}(\mathbb{Q})$ act on $\mathbb{Q}_{\mathrm{B}}(1)$ as $v^{-1}$. There is then a natural action of $\mathrm{GL}\left(H_{\mathrm{B}}^{1}(X)\right) \times \mathbb{G}_{m}$ on $H_{\mathrm{B}}^{r}\left(X^{n}\right)(m)$ for any $r, n$, and $m$. We define $G$ to be the subgroup of $\operatorname{GL}\left(H_{\mathrm{B}}^{1}(X)\right) \times \mathbb{G}_{m}$ fixing all the tensors of the form $c l_{\mathrm{B}}(Z), Z$ an algebraic cycle on some $X^{n}$ (see the Notations).

Consider the comparison isomorphisms

$$
H_{\mathrm{dR}}^{1}(X) \otimes_{k} \mathbb{C} \stackrel{\simeq}{\rightarrow} H^{1}\left(X^{\mathrm{an}}, \mathbb{C}\right) \stackrel{\simeq}{\longleftarrow} H_{\mathrm{B}}^{1}(X) \otimes_{\mathbb{Q}} \mathbb{C} .
$$

The periods $p_{i j}$ of $X$ are defined by the equations

$$
\alpha_{i}=\sum p_{j i} a_{j}
$$

where $\left\{\alpha_{i}\right\}$ and $\left\{a_{i}\right\}$ are bases for $H_{\mathrm{dR}}^{1}(X)$ and $H_{\mathrm{B}}^{1}(X)$ over $k$ and $\mathbb{Q}$ respectively. The field $k\left(p_{i j}\right)$ generated over $k$ by the $p_{i j}$ is independent of the bases chosen.

PROPOSITION 1.6. With the above definitions, the transcendence degree of $k\left(p_{i j}\right)$ over $k$ is $\leq \operatorname{dim}(G)$.

PROOF. We can replace $k$ by its algebraic closure in $\mathbb{C}$, and hence assume that each algebraic cycle on $X_{\mathbb{C}}$ is equivalent to an algebraic cycle on $X$ (see the proof of 1.5). Let $P$ be the functor of $k$-algebras whose value on $R$ is the set of isomorphisms $p: H_{\mathrm{B}}^{1} \otimes_{\mathbb{Q}} R \rightarrow H_{\mathrm{dR}}^{1} \otimes_{k} R$ mapping $c l_{\mathrm{B}}(Z) \otimes 1$ to $c l_{\mathrm{dR}}(Z) \otimes 1$ for all algebraic cycles $Z$ on a power of $X$. When $R=\mathbb{C}$, the comparison isomorphism is such a $p$, and so $P(\mathbb{C})$ is not empty. It is easily seen that $P$ is represented by an algebraic variety that becomes a $G_{k}$-torsor under the obvious action. The bases $\left\{\alpha_{i}\right\}$ and $\left\{a_{i}\right\}$ can be used to identify the points of $P$ with matrices. The matrix $\left(p_{i j}\right)$ is a point of $P$ with coordinates in $\mathbb{C}$, and so the proposition is a consequence of the following easy lemma.

Lemma 1.7. Let $\mathbb{A}^{N}$ be the affine $N$-space over a subfield $k$ of $\mathbb{C}$, and let $z \in \mathbb{A}^{N}(\mathbb{C})$. The transcendence degree of $k\left(z_{1}, \ldots, z_{N}\right)$ over $k$ is the dimension of the Zariski closure of $\{z\}$ in $\mathbb{A}^{N}$.

PROOF. Let $\mathfrak{a}$ be the kernel of the homomorphism $k\left[T_{1}, \ldots, T_{N}\right] \rightarrow k\left[z_{1}, \ldots, z_{N}\right]$ sending $T_{i}$ to $z_{i}$. The Zariski closure of $\{z\}$ in $\mathbb{A}^{N}$ is the zero set $Z(\mathfrak{a})$ of $\mathfrak{a}$, and $Z(\mathfrak{a})$ is a variety over $k$ with function field $k\left(z_{1}, \ldots, z_{N}\right)$. Now $\operatorname{dim}(Z(\mathfrak{a}))=\operatorname{tr}$. deg. $k\left(z_{1}, \ldots, z_{N}\right)$ (standard result). 
REMARK 1.8. If $X$ is an elliptic curve, then $\operatorname{dim} G$ is 2 or 4 according as $X$ has complex multiplication or not. Chudnovsky has shown that

$$
\text { tr. } \operatorname{deg} \cdot k k\left(p_{i j}\right)=\operatorname{dim} G
$$

when $X$ is an elliptic curve with complex multiplication. Does equality hold for all abelian varieties?

One of the main purposes of the seminar was to show that, in the case that $X$ is an abelian variety, (1.5) and (1.6) make sense, and remain true, if "algebraic cycle" is replaced by "Hodge cycle". 


\section{Absolute Hodge cycles; principle B}

\section{Definitions ( $k$ algebraically closed of finite transcendence degree)}

Let $k$ be an algebraically closed field of finite transcendence degree over $\mathbb{Q}$, and let $X$ be a complete smooth variety over $k$. Set

$$
H_{\AA}^{n}(X)(m)=H_{\mathrm{dR}}^{n}(X)(m) \times H_{\mathrm{et}}^{n}(X)(m)
$$

- it is a free $k \times \mathbb{A}_{f}$-module. Corresponding to an embedding $\sigma: k \hookrightarrow \mathbb{C}$, there are canonical isomorphisms

$$
\begin{gathered}
\sigma_{\mathrm{dR}}^{*}: H_{\mathrm{dR}}^{n}(X)(m) \otimes_{k, \sigma} \mathbb{C} \stackrel{\simeq}{\stackrel{\sim}{\rightarrow}} H_{\mathrm{dR}}^{n}(\sigma X)(m) \\
\sigma_{\mathrm{et}}^{*}: H_{\mathrm{et}}^{n}(X)(m) \stackrel{\simeq}{\rightarrow} H_{\mathrm{et}}^{n}(\sigma X)(m)
\end{gathered}
$$

whose product we write $\sigma^{*}$. The diagonal embedding

$$
H_{\sigma}^{n}(X)(m) \hookrightarrow H_{\mathrm{dR}}^{n}(\sigma X)(m) \times H_{\mathrm{et}}^{n}(\sigma X)(m)
$$

induces an isomorphism

$$
H_{\sigma}^{n}(X)(m) \otimes\left(\mathbb{C} \times \mathbb{A}_{f}\right) \stackrel{\simeq}{\rightarrow} H_{\mathrm{dR}}^{n}(\sigma X)(m) \times H_{\mathrm{et}}^{n}(\sigma X)(m)
$$

(product of the comparison isomorphisms, §1). An element $t \in H_{\mathbb{A}}^{2 p}(X)(p)$ is a Hodge cycle relative to $\sigma$ if

(a) $t$ is rational relative to $\sigma$, i.e., $\sigma^{*}(t)$ lies in the rational subspace $H_{\sigma}^{2 p}(X)(p)$ of $H_{\mathrm{dR}}^{2 p}(\sigma X)(p) \times H_{\mathrm{et}}^{2 p}(\sigma X)(p)$

(b) the first component of $t$ lies in $F^{0} H_{\mathrm{dR}}^{2 p}(X)(p) \stackrel{\text { def }}{=} F^{p} H_{\mathrm{dR}}^{2 p}(X)$.

Equivalent condition: $\sigma^{*}(t)$ lies in $H_{\sigma}^{2 p}(X)(p)$ and is of bidegree $(0,0)$. If $t$ is a Hodge cycle relative to every embedding $\sigma: k \hookrightarrow \mathbb{C}$, then it is called an absolute Hodge cycle.

EXAMPLE 2.1. (a) For any algebraic cycle $Z$ on $X, t=\left(c l_{\mathrm{dR}}(Z), c l_{\mathrm{et}}(Z)\right)$ is an absolute Hodge cycle - the Hodge conjecture predicts there are no others. Indeed, for any $\sigma: k \hookrightarrow \mathbb{C}, \sigma^{*}(t)=c l_{\mathrm{B}}(Z)$, and is therefore rational, and it is well-known that $c l_{\mathrm{dR}}(\sigma Z)$ is of bidegree $(p, p)$ in $H_{\mathrm{dR}}^{2 p}(\sigma X)$.

(b) Let $X$ be a complete smooth variety of dimension $d$, and consider the diagonal $\Delta \subset X \times X$. Corresponding to the decomposition

$$
H^{2 d}(X \times X)(d)=\bigoplus_{i=0}^{2 d} H^{2 d-i}(X) \otimes H^{i}(X)(d)
$$

we have

$$
\operatorname{cl}(\Delta)=\sum_{i=0}^{2 d} \pi^{i}
$$

The $\pi^{i}$ are absolute Hodge cycles.

(c) Suppose that $X$ is given with a projective embedding, and let $\gamma \in H_{\mathrm{dR}}^{2}(X)(1) \times$ $H_{\mathrm{et}}^{2}(X)(1)$ be the class of a hyperplane section. The hard Lefschetz theorem states that

$$
x \mapsto \gamma^{d-2 p} \cdot x: H^{2 p}(X)(p) \rightarrow H^{2 d-2 p}(X)(d-p), \quad 2 p \leq d,
$$

is an isomorphism. The class $x$ is an absolute Hodge cycle if and only if $\gamma^{d-2 p} \cdot x$ is an absolute Hodge cycle. 
Loosely speaking, every cycle constructed from a set of absolute Hodge cycles by a canonical rational process will again be an absolute Hodge cycle.

Open Question 2.2. Does there exist a cycle rational for every $\sigma$ but which is not absolutely Hodge?

More generally, consider a family $\left(X_{\alpha}\right)_{\alpha \in A}$ of complete smooth varieties over a field $k$ (as above). Let $(m(\alpha)) \in \mathbb{N}^{(A)},(n(\alpha)) \in \mathbb{N}^{(A)}$, and $m \in \mathbb{Z}$, and write

$$
\begin{aligned}
T_{\mathrm{dR}} & =\left(\bigotimes_{\alpha} H_{\mathrm{dR}}^{m(\alpha)}\left(X_{\alpha}\right)\right) \otimes\left(\bigotimes_{\alpha} H_{\mathrm{dR}}^{n(\alpha)}\left(X_{\alpha}\right)^{\vee}\right)(m) \\
T_{\mathrm{et}} & =\left(\bigotimes_{\alpha} H_{\mathrm{et}}^{m(\alpha)}\left(X_{\alpha}\right)\right) \otimes\left(\bigotimes_{\alpha} H_{\mathrm{et}}^{n(\alpha)}\left(X_{\alpha}\right)^{\vee}\right)(m) \\
T_{\mathrm{A}} & =T_{\mathrm{dR}} \times T_{\mathrm{et}} \\
T_{\sigma} & =\left(\bigotimes_{\alpha} H_{\sigma}^{m(\alpha)}\left(X_{\alpha}\right)\right) \otimes\left(\bigotimes_{\alpha} H_{\sigma}^{n(\alpha)}\left(X_{\alpha}\right)^{\vee}\right)(m) \quad(\sigma: k \hookrightarrow \mathbb{C}) .
\end{aligned}
$$

Then we say that $t \in T_{\mathbb{A}}$ is

$\diamond$ rational relative to $\sigma$ if its image in $T_{\mathbb{A}} \otimes_{k \times \mathbb{A}_{f},(\sigma, 1)} \mathbb{C} \times \mathbb{A}_{f}$ lies in $T_{\sigma}$,

$\diamond$ a Hodge cycle relative to $\sigma$ if it is rational relative to $\sigma$ and its first component lies in $F^{0}$, and

$\diamond \quad$ an absolute Hodge cycle if it is a Hodge cycle relative to every $\sigma$.

Note that, in order for there to exist Hodge cycles in $T_{\mathbb{A}}$, it is necessary that

$$
\sum m(\alpha)-\sum n(\alpha)=2 m \text {. }
$$

EXAMPLE 2.3. Cup product defines maps

$$
T_{\mathbb{A}}^{m, n}(p) \times T_{\mathbb{A}}^{m^{\prime}, n^{\prime}}\left(p^{\prime}\right) \rightarrow T_{\mathbb{A}}^{m+m^{\prime}, n+n^{\prime}}\left(p+p^{\prime}\right),
$$

and hence an element of $T_{\mathbb{A}}^{\vee} \otimes T_{\mathbb{A}}^{\vee} \otimes T_{\mathbb{A}}$, which is an absolute Hodge cycle.

OPEN Question 2.4. Let $t \in F^{0} H_{\mathrm{dR}}^{2 p}(X)(p)$. If $\sigma_{\mathrm{dR}}^{*}(t) \in H_{\sigma}^{2 p}(X)(\sigma)$ for all $\sigma: k \hookrightarrow \mathbb{C}$, is $t$ necessarily the first component of an absolute Hodge cycle?

\section{Basic properties of absolute Hodge cycles}

In order to develop the theory of absolute Hodge cycles, we shall need to use the GaussManin connection (Katz and Oda 1968; Katz 1970; Deligne 1971b). Let $k_{0}$ be a field of characteristic zero and let $S$ be a smooth $k_{0}$-scheme (or the spectrum of a finitely generated field over $\left.k_{0}\right)$. A $k_{0}$-connection on a coherent $\mathcal{O}_{S}$-module $\mathcal{E}$ is a homomorphism of sheaves of abelian groups

$$
\nabla: \mathcal{E} \rightarrow \Omega_{S / k_{0}}^{1} \otimes_{\mathcal{O}_{S}} \mathcal{E}
$$

such that

$$
\nabla(f e)=d f \otimes e+f \nabla(e)
$$


for local sections $f$ of $\mathcal{O}_{S}$ and $e$ of $\mathcal{E}$. The kernel of $\nabla, \mathcal{E}^{\nabla}$, is the sheaf of horizontal sections of $(\mathcal{E}, \nabla)$. Every $k_{0}$-connection $\nabla$ can be extended to a homomorphism of abelian sheaves,

$$
\begin{aligned}
\nabla_{n}: \Omega_{S / k_{0}}^{n} \otimes_{\mathcal{O}_{S}} \mathcal{E} & \rightarrow \Omega_{S / k_{0}}^{n+1} \otimes_{\mathcal{O}_{S}} \mathcal{E}, \\
\omega \otimes e & \mapsto d \omega \otimes e+(-1)^{n} \omega \wedge \nabla(e)
\end{aligned}
$$

and $\nabla$ is said to be integrable if $\nabla_{1} \circ \nabla=0$. Moreover, $\nabla$ gives rise to an $\mathcal{O}_{S}$-linear map

$$
D \mapsto \nabla_{D}: \operatorname{Der}\left(S / k_{0}\right) \rightarrow \operatorname{End}_{k_{0}}(\mathcal{E})
$$

where $\nabla_{D}$ is the composite

$$
\mathcal{E} \stackrel{\nabla}{\rightarrow} \Omega_{S / k_{0}}^{1} \otimes_{\mathcal{O}_{S}} \mathcal{E} \stackrel{D \otimes 1}{\rightarrow} \mathcal{O}_{S} \otimes_{\mathcal{O}_{S}} \mathcal{E} \simeq \mathcal{E} .
$$

Note that $\nabla_{D}(f e)=D(f) e+f \nabla_{D}(e)$. One checks that $\nabla$ is integrable if and only if $D \mapsto \nabla_{D}$ is a Lie algebra homomorphism.

Now consider a proper smooth morphism $\pi: X \rightarrow S$ of smooth varieties, and write $\mathcal{H}_{\mathrm{dR}}^{n}(X / S)$ for $\mathbb{R}^{n} \pi_{*}\left(\Omega_{X / S}^{\bullet}\right)$. This is a locally free sheaf of $\mathcal{O}_{S}$-modules with a canonical connection $\nabla$, called the Gauss-Manin connection, which is integrable. It therefore defines a Lie algebra homomorphism

$$
\operatorname{Der}\left(S / k_{0}\right) \rightarrow \operatorname{End}_{k_{0}}\left(\mathcal{H}_{\mathrm{dR}}^{n}(X / S)\right) .
$$

If $k_{0} \hookrightarrow k_{0}^{\prime}$ is an inclusion of fields and $X^{\prime} / S^{\prime}=(X / S) \otimes_{k_{0}} k_{0}^{\prime}$, then the Gauss-Manin connection on $\mathcal{H}_{\mathrm{dR}}^{n}\left(X^{\prime} / S^{\prime}\right)$ is $\nabla \otimes 1$. In the case that $k_{0}=\mathbb{C}$, the relative form of Serre's GAGA theorem (Serre 1956) shows that $\mathcal{H}_{\mathrm{dR}}^{n}(X / S)^{\text {an }} \simeq \mathcal{H}_{\mathrm{dR}}^{n}\left(X^{\mathrm{an}} / X^{\mathrm{an}}\right)$ and $\nabla$ gives rise to a connection $\nabla^{\text {an }}$ on $\mathcal{H}_{\mathrm{dR}}^{n}\left(X^{\text {an }} / S^{\text {an }}\right)$. The relative Poincaré lemma shows that

$$
\left(R^{n} \pi_{*} \mathbb{C}\right) \otimes \mathcal{O}_{S^{\mathrm{an}}} \stackrel{\simeq}{\rightarrow} \mathcal{H}_{\mathrm{dR}}^{n}\left(X^{\mathrm{an}} / S^{\mathrm{an}}\right),
$$

and it is known that $\nabla^{\text {an }}$ is the unique connection such that

$$
R^{n} \pi_{*} \mathbb{C} \stackrel{\simeq}{\rightarrow} \mathcal{H}_{\mathrm{dR}}^{n}\left(X^{\mathrm{an}} / S^{\mathrm{an}}\right)^{\nabla^{\mathrm{an}}} .
$$

Proposition 2.5. Let $k_{0} \subset \mathbb{C}$ have finite transcendence degree over $\mathbb{Q}$, and let $X$ be a complete smooth variety over a field $k$ that is finitely generated over $k_{0}$. Let $\nabla$ be the Gauss-Manin connection on $\mathcal{H}_{d R}^{n}(X)$ relative to the composite $X \rightarrow \operatorname{Spec} k \rightarrow \operatorname{Spec} k_{0}$. If $t \in H_{d R}^{n}(X)$ is rational relative to all embeddings of $k$ into $\mathbb{C}$, then $t$ is horizontal for $\nabla$, i.e., $\nabla t=0$.

Proof. Choose a regular $k_{0}$-algebra $A$ of finite-type and a smooth proper map $\pi: X_{A} \rightarrow$ Spec $A$ whose generic fibre is $X \rightarrow$ Spec $k$ and which is such that $t$ extends to an element of $\Gamma\left(\operatorname{Spec} A, \mathcal{H}_{\mathrm{dR}}^{n}(X / \operatorname{Spec} A)\right.$. After a base change relative to $k_{0} \hookrightarrow \mathbb{C}$, we obtain maps

$$
X_{S} \rightarrow S \rightarrow \operatorname{Spec} \mathbb{C}, \quad S=\operatorname{Spec} A_{\mathbb{C}}
$$

and a global section $t^{\prime}=t \otimes 1$ of $\mathcal{H}_{\mathrm{dR}}^{n}\left(X_{S}^{\text {an }} / S^{\text {an }}\right)$. We have to show that $(\nabla \otimes 1) t^{\prime}=0$, or equivalently, that $t^{\prime}$ is a global section of $\mathcal{H}^{n}\left(X_{S}^{\text {an }}, \mathbb{C}\right) \stackrel{\text { def }}{=} R^{n} \pi_{*}^{\text {an }} \mathbb{C}$. 
An embedding $\sigma: k \hookrightarrow \mathbb{C}$ gives rise to an injection $A \hookrightarrow \mathbb{C}$ (i.e., a generic point of $\operatorname{Spec} A$ in the sense of Weil) and hence a point $s$ of $S$. The hypotheses show that, at each of these points, $t(s) \in H^{n}\left(X_{s}^{\mathrm{an}}, \mathbb{Q}\right) \subset H_{\mathrm{dR}}^{n}\left(X_{s}^{\mathrm{an}}\right)$. Locally on $S, H_{\mathrm{dR}}^{n}\left(X_{s}^{\mathrm{an}} / S^{\mathrm{an}}\right)$ will be the sheaf of holomorphic sections of the trivial bundle $S \times \mathbb{C}^{n}$ and $\mathcal{H}^{n}\left(X^{\text {an }}, \mathbb{C}\right)$ will be the sheaf of locally constant sections. Thus, locally, $t^{\prime}$ is a function

$$
s \mapsto\left(t_{1}(s), \ldots, t_{m}(s)\right): S \rightarrow S \times \mathbb{C}^{m} .
$$

Each $t_{i}(s)$ is a holomorphic function which, by hypothesis, takes real (even rational) values on a dense subset of $S$. It is therefore constant.

REMARK 2.6. In the situation of (2.5), assume that $t \in H_{\mathrm{dR}}^{n}(X)$ is rational relative to one $\sigma$ and horizontal for $\nabla$. An argument similar to the above then shows that $t$ is rational relative to all embeddings that agree with $\sigma$ on $k_{0}$.

COROLlaRY 2.7. Let $k_{0} \subset k$ be algebraically closed fields of finite transcendence degree over $\mathbb{Q}$, and let $X$ be a complete smooth variety over $k_{0}$. If $t \in H_{d R}^{n}\left(X_{k}\right)$ is rational relative to all $\sigma: k \hookrightarrow \mathbb{C}$, then it is defined over $k_{0}$, i.e., it is in the image of $H_{d R}^{n}(X) \rightarrow H_{d R}^{n}\left(X_{k}\right)$.

Proof. Let $k^{\prime}$ be a subfield of $k$ which is finitely generated over $k_{0}$ and such that $t \in$ $H_{\mathrm{dR}}^{n}\left(X \otimes_{k_{0}} k^{\prime}\right)$. The hypothesis implies that $\nabla t=0$ where $\nabla$ is the Gauss-Manin connection for $X_{k^{\prime}} \rightarrow \operatorname{Spec} k^{\prime} \rightarrow$ Spec $k_{0}$. Thus, for any $D \in \operatorname{Der}\left(k^{\prime} / k_{0}\right), \nabla_{D}(t)=0$. But $X_{k^{\prime}}$ arises from a variety over $k_{0}$, and so $\operatorname{Der}\left(k^{\prime} / k_{0}\right)$ acts on $H_{\mathrm{dR}}^{n}\left(X_{k^{\prime}}\right)=H_{\mathrm{dR}}^{n}(X) \otimes_{k_{0}} k^{\prime}$ through $k^{\prime}$, i.e., $\nabla_{D}=1 \otimes D$. Thus the corollary follows from the next well-known lemma.

LEMMA 2.8. Let $k_{0} \subset k^{\prime}$ be as above, and let $V=V_{0} \otimes_{k_{0}} k^{\prime}$, where $V_{0}$ is a vector space over $k_{0}$. If $t \in V$ is fixed (i.e., killed) by all derivations of $k^{\prime} / k_{0}$, then $t \in V_{0}$.

Let $C_{\mathrm{AH}}^{p}(X)$ be the subset of $H_{\mathbb{A}}^{2 p}(X)(p)$ of absolute Hodge cycles. It is a finitedimensional vector space over $\mathbb{Q}$.

PROpOSITION 2.9. Let $k$ be an algebraically closed field of finite transcendence degree over $\mathbb{Q}$.

(a) For any smooth complete variety $X$ defined over an algebraically closed subfield $k_{0}$ of $k$, the canonical map

$$
H_{\mathbb{A}}^{2 p}(X)(p) \rightarrow H_{\mathbb{A}}^{2 p}\left(X_{k}\right)(p)
$$

induces an isomorphism

$$
C_{\mathrm{AH}}^{p}(X) \rightarrow C_{\mathrm{AH}}^{p}\left(X_{k}\right) .
$$

(b) Let $X_{0}$ be a smooth complete variety defined over a subfield $k_{0}$ of $k$ whose algebraic closure is $k$, and let $X=X_{0} \otimes_{k_{0}} k$. Then $\operatorname{Gal}\left(k / k_{0}\right)$ acts on $C_{\mathrm{AH}}^{p}(X)$ through a finite quotient.

Proof. (a) The map is injective, and a cycle on $X$ is absolutely Hodge if and only if it is absolutely Hodge on $X_{k}$, and so it remains to show that an absolute Hodge cycle $t$ on $X_{k}$ arises from a cycle on $X$. But (2.7) shows that $t_{\mathrm{dR}}$ arises from an element of $H_{\mathrm{dR}}^{2 p}(X)(p)$, and $H_{\mathrm{et}}^{2 p}(X)(p) \rightarrow H_{\mathrm{et}}^{2 p}\left(X_{k}\right)(p)$ is an isomorphism.

(b) It is obvious that the action of $\operatorname{Gal}\left(k / k_{0}\right)$ on $H_{\mathrm{dR}}^{2 p}(X)(p) \times H_{\mathrm{et}}^{2 p}(X)(p)$ stabilizes $C_{\mathrm{AH}}^{p}(X)$. We give three proofs that it factors through a finite quotient. 
(i) Note that $C_{\mathrm{AH}}^{p}(X) \rightarrow H_{\mathrm{dR}}^{2 p}(X)$ is injective. Clearly, $H_{\mathrm{dR}}^{2 p}(X)=\bigcup H_{\mathrm{dR}}^{2 p}\left(X_{0} \otimes k_{i}\right)$ where the $k_{i}$ run over over the finite extensions of $k_{0}$ contained in $k$. Thus, all elements of a finite generating set for $C_{\mathrm{AH}}^{p}(X)$ lie in $H_{\mathrm{dR}}^{2 p}\left(X_{0} \otimes k_{i}\right)$ for some $i$.

(ii) Note that $C_{\mathrm{AH}}^{p}(X) \rightarrow H^{2 p}\left(X_{\mathrm{et}}, \mathbb{Q}_{\ell}\right)(p)$ is injective for all $\ell$. The subgroup $H$ of $\operatorname{Gal}\left(k / k_{0}\right)$ fixing $C_{\mathrm{AH}}^{P}(X)$ is closed. Thus, the quotient of $\operatorname{Gal}\left(k / k_{0}\right)$ by $H$ is a profinite group, which is countable because it is a finite subgroup of $\mathrm{GL}_{m}(\mathbb{Q})$ for some $m$. It follows that it is finite (the Cantor diagonalization argument shows that an infinite profinite group is uncountable).

(iii) A polarization of $X$ gives a positive definite form on $C_{\mathrm{AH}}^{p}(X)$, which is stable under $\operatorname{Gal}\left(k / k_{0}\right)$. This shows that the action factors through a finite quotient.

REMARK 2.10. (a) The above results remain valid for a family of varieties $\left(X_{\alpha}\right)_{\alpha}$ rather than a single $X$.

(b) Proposition 2.9 would remain true if we had defined an absolute Hodge cycle to be an element $t$ of $F^{0} H_{\mathrm{dR}}^{2 p}(X)(p)$ such that, for all $\sigma: k \hookrightarrow \mathbb{C}, \sigma_{\mathrm{dR}}^{*}(t) \in H_{\sigma}^{2 p}(X)$.

\section{Definitions (arbitrary $k$ )}

Proposition 2.9 allows us to define the notion of an absolute Hodge cycle on any smooth complete variety $X$ over a field of characteristic zero. When $k$ is algebraically closed, we choose a model $X_{0} / k_{0}$ of $X_{0}$ over an algebraically closed subfield $k_{0}$ of $k$ of finite transcendence degree over $\mathbb{Q}$, and we define $t \in H_{\mathbb{A}}^{2 p}(X)(p)$ to be an absolute Hodge cycle if it lies in the subspace $H_{\mathbb{A}}^{2 p}\left(X_{0}\right)(p)$ of $H_{\mathbb{A}}^{2 p}(X)(p)$ and is an absolute Hodge cycle there. The proposition shows that this definition is independent of the choice of $k_{0}$ and $X_{0}$. (This definition is forced on us if we want (2.9a) to hold without restriction on the transcendence degrees of $k$ and $k_{0}$.) When $k$ is not algebraically closed, we choose an algebraic closure $\bar{k}$ of it, and define an absolute Hodge cycle on $X$ to be an absolute Hodge cycle on $X \otimes_{k} \bar{k}$ that is fixed by $\operatorname{Gal}(\bar{k} / k)$.

One can show (assuming the axiom of choice) that if $k$ is algebraically closed and of cardinality not greater than that of $\mathbb{C}$, then an element $t$ of $H_{\mathrm{dR}}^{2 p}(X)(p) \times H_{\mathrm{et}}^{2 p}(X)(p)$ is an absolute Hodge cycle if it is rational relative to all embeddings $\sigma: k \hookrightarrow \mathbb{C}$ and $t_{\mathrm{dR}} \in$ $F^{0} H_{\mathrm{dR}}^{2 p}(X)(p)$. If $k=\mathbb{C}$, then the first condition has to be checked only for isomorphisms of $\mathbb{C}$. When $k \subset \mathbb{C}$, we define a Hodge cycle to be a cohomology class that is Hodge relative to the inclusion $k \hookrightarrow \mathbb{C}$.

\section{Statement of the main theorem}

MAIN THEOREM 2.11. Let $X$ be an abelian variety over an algebraically closed field $k$, and let $t \in H_{\mathbb{A}}^{2 p}(X)(p)$. If $t$ is a Hodge cycle relative to one embedding $\sigma: k \hookrightarrow \mathbb{C}$, then it is a Hodge cycle relative to every embedding, i.e., it is an absolute Hodge cycle.

The proof will occupy $\S \S 2-6$ of the notes.

\section{Principle B}

We begin with a result concerning families of varieties parametrized by smooth algebraic varieties over $\mathbb{C}$. Let $\pi: X \rightarrow S$ be a proper smooth map of smooth varieties over $\mathbb{C}$ with $S$ 
connected. We set

$$
\mathcal{H}_{\mathrm{et}}^{n}(X / S)(m)=\overleftarrow{r}_{r}^{\lim }\left(R^{n} \pi_{\mathrm{et}}^{*} \mu_{r}^{\otimes m}\right) \otimes_{\mathbb{Z}} \mathbb{Q}
$$

and

$$
\mathcal{H}_{\mathbb{A}}^{n}(X / S)(m)=\mathcal{H}_{\mathrm{dR}}^{n}(X / S)(m) \times \mathcal{H}_{\mathrm{et}}^{n}(X / S)(m) .
$$

THEOREM 2.12 (PRINCIPLE B). Let $t$ be a global section of $\mathcal{H}_{\mathbb{A}}^{2 p}(X / S)(p)$ such that $\nabla t_{d R}=0$. If $\left(t_{d R}\right)_{s} \in F^{0} H_{d R}^{2 p}\left(X_{S}\right)(p)$ for all $s \in S$ and $t_{s}$ is an absolute Hodge cycle in $H_{\mathbb{A}}^{2 p}\left(X_{s}\right)(p)$ for one $s$, then $t_{s}$ is an absolute Hodge cycle for all $s$.

Proof. Suppose that $t_{s}$ is an absolute Hodge cycle for $s=s_{1}$, and let $s_{2}$ be a second point of $S$. We have to show that $t_{S_{2}}$ is rational relative to every isomorphism $\sigma: \mathbb{C} \rightarrow \mathbb{C}$. On applying $\sigma$, we obtain a morphism $\sigma \pi: \sigma X \rightarrow \sigma S$ and a global section $\sigma t$ of $\mathcal{H}_{\mathbb{A}}^{2 p}(X / S)(p)$. We know that $\sigma(t)_{\sigma s_{1}}$ is rational, and we have to show that $\sigma(t)_{\sigma s_{2}}$ is rational. Clearly, $\sigma$ only translates the problem, and so we can omit it.

First consider the component $t_{\mathrm{dR}}$ of $t$. By assumption, $\nabla t_{\mathrm{dR}}=0$, and so $t_{\mathrm{dR}}$ is a global section of $\mathcal{H}^{2 p}\left(X^{\text {an }}, \mathbb{C}\right)$. Since it is rational at one point, it must be rational at every point.

Next consider $t_{\mathrm{et}}$. As $\mathcal{H}_{\mathrm{B}}^{2 p}(X / S)(p) \stackrel{\text { def }}{=} R^{2 p} \pi_{*}^{\text {an }} \mathbb{Q}(p)$ and $\mathcal{H}_{\mathrm{et}}^{2 p}(X / S)$ are local systems, for any point $s \in S$ there are isomorphisms

$$
\begin{aligned}
\Gamma\left(S, \mathcal{H}_{\mathrm{B}}^{2 p}(X / S)(p)\right) \stackrel{\stackrel{\simeq}{\rightarrow}}{\stackrel{\simeq}{2 p}} H_{\mathrm{B}}\left(X_{S}\right)(p)^{\pi_{1}(S, s)} \\
\Gamma\left(S, \mathcal{H}_{\mathrm{et}}^{2 p}(X / S)(p)\right) \stackrel{\simeq}{\rightarrow} H_{\mathrm{et}}^{2 p}\left(X_{S}\right)(p)^{\pi_{1}(S, s)} .
\end{aligned}
$$

Consider

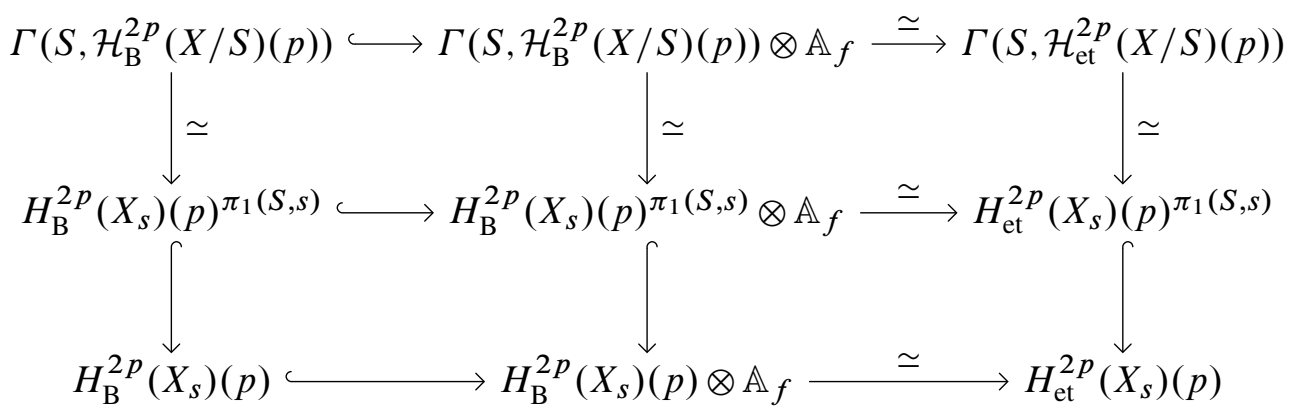

We have $t_{\mathrm{et}} \in \Gamma\left(S, \mathcal{H}_{\mathrm{et}}^{2 p}(X / S)(p)\right)$ and are told that its image in $H_{\mathrm{et}}^{2 p}\left(X_{S_{1}}\right)(p)$ lies in $H_{\mathrm{B}}^{2 p}\left(X_{S_{1}}\right)(p)$. On applying the next lemma (with $Z=\mathbb{A}$ and $z=1$ ), we find that $t_{\mathrm{et}}$ lies in $\Gamma\left(S, \mathcal{H}_{\mathrm{B}}^{2 p}(X / S)(p)\right)$, and is therefore in $H_{\mathrm{B}}^{2 p}\left(X_{S}\right)(p)$ for all $s$.

Lemma 2.13. Let $W \hookrightarrow V$ be an inclusion of vector spaces. Let $Z$ be a third vector space and let $z$ be a nonzero element of $Z$. Embed $V$ in $V \otimes Z$ by $v \mapsto v \otimes z$. Then

$$
(W \otimes Z) \cap V=W \quad \text { (inside } V \otimes Z) .
$$

Proof. Choose a basis $\left(e_{i}\right)_{i \in I}$ for $W$ and extend it to a basis $\left(e_{i}\right)_{I \sqcup J}$ for $V$. Every $x \in V \otimes Z$ has a unique expression

$$
x=\sum_{i \in I \sqcup J} e_{i} \otimes z_{i}, \quad\left(z_{i} \in Z, \text { finite sum }\right) .
$$

If $x \in W \otimes Z$, then $z_{i}=0$ for $i \notin I$, and if $x \in V$, then $z_{i}=z$ for all $i$. 
REMARK 2.14. The assumption in the theorem that $\left(t_{\mathrm{dR}}\right)_{s} \in F^{0} H_{\mathrm{dR}}^{2 p}\left(X_{s}\right)(p)$ for all $s$ is unnecessary: it is implied by the condition that $\nabla t_{\mathrm{dR}}=0$ (Deligne 1971a, 4.1.2, Théorème de la partie fixe).

We shall need a slight generalization of Theorem 2.12.

THEOREM 2.15. Let $\pi: X \rightarrow S$ again be a smooth proper map of smooth varieties over

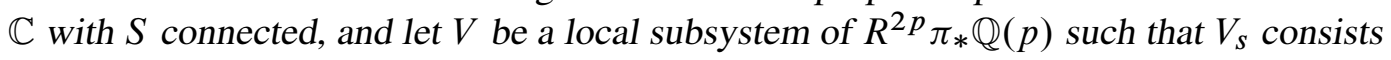
of $(0,0)$-cycles for all $s$ and consists of absolute Hodge cycles for at least one $s$. Then $V_{s}$ consists of absolute Hodge cycles for all $s$.

PROOF. If $V$ is constant, so that every element of $V_{s}$ extends to a global section, then this is a consequence of Theorem 2.12, but the following argument reduces the proof of the general case to that case.

At each point $s \in S, R^{2 p} \pi_{*} \mathbb{Q}(p)_{s}$ has a Hodge structure. Moreover, $R^{2 p_{\pi_{*}}} \mathbb{Q}(p)$ has a polarization, i.e., there is a form

$$
\psi: R^{2 p} \pi_{*} \mathbb{Q}(p) \times R^{2 p} \pi_{*} \mathbb{Q}(p) \rightarrow \mathbb{Q}(-p)
$$

which at each point defines a polarization on the Hodge structure $R^{2 p} \pi_{*} \mathbb{Q}(p)_{s}$. On

$$
R^{2 p} \pi_{*} \mathbb{Q}(p) \cap\left(R^{2 p} \pi_{*} \mathbb{C}(p)\right)^{0,0}
$$

the form is symmetric, bilinear, rational, and positive definite. Since the action of $\pi_{1}\left(S, s_{0}\right)$ preserves the form, the image of $\pi_{1}\left(S, s_{0}\right)$ in $\operatorname{Aut}\left(V_{s_{0}}\right)$ is finite. Thus, after passing to a finite covering of $S$, we can assume that $V$ is constant.

REMARK 2.16. Both Theorem 2.12 and Theorem 2.15 generalize, in an obvious way, to families $\pi_{\alpha}: X_{\alpha} \rightarrow S$. 


\section{Mumford-Tate groups; principle A}

\section{Characterizing subgroups by their fixed tensors}

Let $G$ be a reductive algebraic group over a field $k$ of characteristic zero, and let $\left(V_{\alpha}\right)_{\alpha \in A}$ be a faithful family of finite-dimensional representations over $k$ of $G$, so that $G \rightarrow \prod \operatorname{GL}\left(V_{\alpha}\right)$ is injective. For all $m, n \in \mathbb{N}^{(A)}$, we can form

$$
T^{m, n}=\bigotimes_{\alpha} V_{\alpha}^{\otimes m(\alpha)} \otimes \bigotimes_{\alpha}\left(V_{\alpha}^{\vee}\right)^{\otimes n(\alpha)},
$$

which is again a finite-dimensional representation of $G$. For an algebraic subgroup $H$ of $G$, we write $H^{\prime}$ for the subgroup of $G$ fixing all tensors that occur in some $T^{m, n}$ and are fixed by $H$. Clearly, $H \subset H^{\prime}$, and we shall need criteria guaranteeing their equality.

Proposition 3.1. The notations are as above.

(a) Every finite-dimensional representation of $G$ is contained in a direct sum of representations $T^{m, n}$.

(b) (Chevalley's Theorem). Every subgroup $H$ of $G$ is the stabilizer of a line $D$ in some finite-dimensional representation of $G$.

(c) If $H$ is reductive, or if $X_{k}(G) \rightarrow X_{k}(H)$ is surjective, then $H=H^{\prime}$. (Here $X_{k}(G)$ denotes $\operatorname{Hom}_{k}\left(G, \mathbb{G}_{m}\right)$, so the hypotheses is that every $k$-character of $H$ extends to a $k$-character of $G$.)

Proof. ${ }^{4}$ (a) Let $W$ be a representation of $G$, and let $W_{0}$ denote the underlying vector space of $W$ with $G$ acting trivially (i.e., $g w=w$, all $g \in G, w \in W$ ). Then $G \times W \rightarrow W$ defines a map $W \rightarrow W_{0} \otimes k[G]$ which is $G$-equivariant (Waterhouse 1979, 3.5). Since $W_{0} \otimes k[G] \approx k[G]^{\operatorname{dim} W}$, it suffices to prove (a) for the regular representation. There is a finite sum $V=\oplus V_{\alpha}$ such that $G \rightarrow \mathrm{GL}(V)$ is injective (because $G$ is noetherian). The map

$$
\mathrm{GL}(V) \rightarrow \operatorname{End}(V) \times \operatorname{End}\left(V^{\vee}\right)
$$

identifies $\operatorname{GL}(V)$ (and hence $G$ ) with a closed subvariety of $\operatorname{End}(V) \times \operatorname{End}\left(V^{\vee}\right)$ (ibid.). There is therefore a surjection

$$
\operatorname{Sym}(\operatorname{End}(V)) \times \operatorname{Sym}\left(\operatorname{End}\left(V^{\vee}\right)\right) \rightarrow k[G],
$$

where Sym denotes the symmetric algebra, and (a) now follows from the fact that representations of reductive groups in characteristic zero are semisimple (see Deligne and Milne 1982, $\S 2)$.

(b) Let $I$ be the ideal of regular functions on $G$ that are zero on $H$. Then, in the regular representation of $G$ on $k[G], H$ is the stabilizer of $I$. There exists a finite-dimensional subspace $V$ of $k[G]$ that is $G$-stable and contains a generating set for $I$ (Waterhouse 1979, 3.3). Then $H$ is the stabilizer of the subspace $I \cap V$ in $V$, and hence of $\bigwedge^{d}(I \cap V)$ in $\bigwedge^{d} V$, where $d=\operatorname{dim}_{k}(I \cap V)$.

(c) According to (b), $H$ is the stabilizer of a line $D$ in some representation $V$ of $G$, which (according to (a)) can be taken to be a direct sum of $T^{m, n}$ 's.

\footnotetext{
${ }^{4}$ Added(jsm): For a detailed proof of (a), see Milne, Algebraic Groups, 2017, 4.14, 22.42; for a detailed proof of (b), see ibid. 4.27
} 
If $H$ is reductive, then $V=W \oplus D$ for some $H$-stable $W$ and $V^{\vee}=W^{\vee} \oplus D^{\vee}$. Now $H$ is the group fixing a generator of $D \otimes D^{\vee}$ in $V \otimes V^{\vee}$.

If every $k$-character of $H$ extends to a $k$-character of $G$, then the one-dimensional representation of $H$ on $D$ can be regarded as the restriction to $H$ of a representation of $G$. Now $H$ is the group fixing a generator of $D \otimes D^{\vee}$ in $V \otimes D^{\vee}$.

REMARK 3.2. (a) It is clearly necessary to have some condition on $H$ in order to have $H^{\prime}=H$. For example, let $B$ be a Borel subgroup of a reductive group $G$, and let $v \in V$ be fixed by $B$. Then $g \mapsto g v$ defines a map of algebraic varieties $G / B \rightarrow V$, which must be constant because $G / B$ is complete and $V$ is affine. Thus, $v$ is fixed by $G$, and so $B^{\prime}=G$.

However, the above argument proves the following: let $H^{\prime}$ be the group fixing all tensors fixed by $G$ occurring in any representation of $G$ (equivalently, any representation occurring as a subquotient of some $T^{m, n}$ ); then $H=H^{\prime}$.

(b) In fact, in all our applications of (3.1c), $H$ will be the Mumford-Tate group of a polarizable Hodge structure, and hence will be reductive. However, the Mumford-Tate groups of mixed Hodge structures (even polarizable) will not in general be reductive, but will satisfy the second condition in (3.1c) (with $G=\mathrm{GL}$ ).

(c) The theorem of Haboush (Demazure 1976) can be used to show that the second form of (3.1c) holds when $k$ has nonzero characteristic.

(d) In (3.1c) it suffices to require that $X_{k}(G) \rightarrow X_{k}(H)$ has finite cokernel, i.e., a nonzero multiple of each $k$-character of $H$ extends to a $k$-character of $G$.

\section{Hodge structures}

Let $V$ be a finite-dimensional vector space over $\mathbb{Q}$. A $\mathbb{Q}$-rational Hodge structure of weight $n$ on $V$ is a decomposition $V_{\mathbb{C}}=\bigoplus_{p+q=n} V^{p, q}$ such that $V^{q, p}$ is the complex conjugate of $V^{p, q}$. Such a structure determines a cocharacter

$$
\mu: \mathbb{G}_{m} \rightarrow \mathrm{GL}\left(V_{\mathbb{C}}\right)
$$

such that

$$
\mu(z) v^{p, q}=z^{-p} v^{p, q}, \quad v^{p, q} \in V^{p, q} .
$$

The complex conjugate $\overline{\mu(z)}$ of $\mu(z)$ has the property $\overline{\mu(z)} \cdot v^{p, q}=\bar{z}^{-q} v^{p, q}$. Since $\mu(z)$ and $\overline{\mu(z)}$ commute, their product determines a homomorphism of real algebraic groups

$$
h: \mathbb{C}^{\times} \rightarrow \mathrm{GL}\left(V_{\mathbb{R}}\right), \quad h(z) v^{p, q}=z^{-p \bar{z}^{-q} v^{p, q} .}
$$

Conversely, a homomorphism $h: \mathbb{C}^{\times} \rightarrow \mathrm{GL}\left(V_{\mathbb{R}}\right)$ whose restriction to $\mathbb{R}^{\times}$is $r \mapsto r^{-n} \cdot \mathrm{id}_{V}$ defines a Hodge structure of weight $n$ on $V$.

Let $F^{p} V=\bigoplus_{p^{\prime} \geq p} V^{p^{\prime}, q^{\prime}}$, so that

$$
\cdots \supset F^{p} V \supset F^{p+1} V \supset \cdots
$$

is a decreasing (Hodge) filtration on $V_{\mathbb{C}}$.

Let $\mathbb{Q}(1)$ denote the vector space $\mathbb{Q}$ with the Hodge structure for which $\mathbb{Q}(1)_{\mathbb{C}}=$ $\mathbb{Q}(1)^{-1,-1}$. It has weight -2 and $h(z) \cdot 1=z \bar{z} \cdot 1$. For any integer $m$,

$$
\mathbb{Q}(m) \stackrel{\text { def }}{=} \mathbb{Q}(1)^{\otimes m}=\mathbb{Q}(m)^{-m,-m}
$$

has weight $-2 m$. (Strictly speaking, we should define $\mathbb{Q}(1)=2 \pi i \mathbb{Q} \ldots$. ) 
REMARK 3.3. The notation $h(z) \cdot v^{p, q}=z^{-p} \bar{z}^{-q} v^{p, q}$ is the negative of that used in Deligne 1971b, Saavedra Rivano 1972, and elsewhere. It is perhaps justified by the following. Let $A$ be an abelian variety over $\mathbb{C}$. The exact sequences

$$
0 \rightarrow \operatorname{Lie}\left(A^{\vee}\right)^{\vee} \rightarrow H_{1}(A, \mathbb{C}) \rightarrow \operatorname{Lie}(A) \rightarrow 0
$$

and

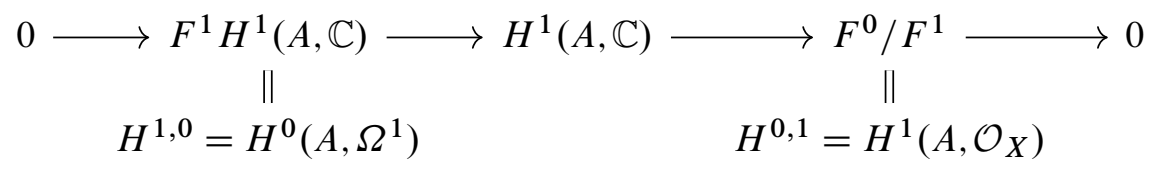

are canonically dual. Since $H^{1}(A, \mathbb{C})$ has a natural Hodge structure of weight 1 with (1,0)-component $H^{0}\left(\Omega^{1}\right), H_{1}(A, \mathbb{C})$ has a natural Hodge structure of weight -1 with $(-1,0)$-component $\operatorname{Lie}(A)$. Thus $h(z)$ acts on $\operatorname{Lie}(A)$, the tangent space to $A$ at zero, as multiplication by $z$.

\section{Mumford-Tate groups}

Let $V$ be a $\mathbb{Q}$-vector space with Hodge structure $h$ of weight $n$. For $m_{1}, m_{2} \in \mathbb{N}$ and $m_{3} \in \mathbb{Z}$, $T=V^{\otimes m_{1}} \otimes V^{\vee \otimes m_{2}} \otimes \mathbb{Q}(1)^{\otimes m_{3}}$ has a Hodge structure of weight $\left(m_{1}-m_{2}\right) n-2 m_{3}$. An element of $T_{\mathbb{C}}$ is said to be rational of bidegree $(p, q)$ if it lies in $T \cap T^{p, q}$. We let $v \in \mathbb{G}_{m}$ act on $\mathbb{Q}(1)$ as $v^{-1}$. The action of $\mathrm{GL}(V)$ on $V$ and the action of $\mathbb{G}_{m}$ on $\mathbb{Q}(1)$ define an action of GL $(V) \times \mathbb{G}_{m}$ on $T$. The Mumford-Tate group $G$ of $(V, h)$ is the subgroup of $\mathrm{GL}(V) \times \mathbb{G}_{m}$ fixing all rational tensors of type $(0,0)$ belonging to any $T$. Thus the projection on the first factor identifies $G(\mathbb{Q})$ with the set of $g \in \mathrm{GL}(V)$ for which there exists a $v(g) \in \mathbb{Q}^{\times}$with the property that $g t=v(g)^{p} t$ for all $t \in V^{\otimes m_{1}} \otimes V^{\vee \otimes m_{2}}$ of type $(p, p)$.

Proposition 3.4. The group $G$ is the smallest algebraic subgroup of $\mathrm{GL}(V) \times \mathbb{G}_{m}$ defined over $\mathbb{Q}$ for which $\mu\left(\mathbb{G}_{m}\right) \subset G_{\mathbb{C}}$.

Proof. Let $H$ be the intersection of all $\mathbb{Q}$-rational subgroups of $\operatorname{GL}(V) \times \mathbb{G}_{m}$ that, over $\mathbb{C}$, contain $\mu\left(\mathbb{G}_{m}\right)$. For any $t \in T, t$ is of type $(0,0)$ if and only if it is fixed by $\mu\left(\mathbb{G}_{m}\right)$ or, equivalently, it is fixed by $H$. Thus $G=H^{\prime}$ in the notation of (3.1), and the next lemma completes the proof.

LEMMA 3.5. With $H$ as above, every $\mathbb{Q}$-character of $H$ extends to a $\mathbb{Q}$-character of $\mathrm{GL}(V) \times \mathbb{G}_{m}$.

Proof. Let $\chi: H \rightarrow \mathrm{GL}(W)$ be a representation of dimension one defined over $\mathbb{Q}$, i.e., a $\mathbb{Q}$-character. The restriction of the representation to $\mathbb{G}_{m}$ is isomorphic to $\mathbb{Q}(n)$ for some $n$. After tensoring $W$ with $\mathbb{Q}(-n)$, we can assume that $\chi \circ \mu=1$, i.e., $\mu\left(\mathbb{G}_{m}\right)$ acts trivially. But then $H$ must act trivially, and the trivial character extends to the trivial character.

Proposition 3.6. If $V$ is polarizable, then $G$ is reductive.

Proof. Choose an $i$ and write $C=h(i)$ ( $C$ is often called the Weil operator). For $v^{p, q} \in$ $V^{p, q}, C v^{p, q}=i^{-p+q} v^{p, q}$, and so $C^{2}$ acts as $(-1)^{n}$ on $V$, where $n=p+q$ is the weight of $V$. 
Recall that a polarization $\psi$ of $V$ is a morphism $\psi: V \times V \rightarrow \mathbb{Q}(-n)$ such that the real-valued form $\psi(x, C y)$ on $V_{\mathbb{R}}$ is symmetric and positive definite. Under the canonical isomorphism

$$
\operatorname{Hom}(V \otimes V, \mathbb{Q}(-n)) \rightarrow V^{\vee} \otimes V^{\vee}(-n),
$$

$\psi$ corresponds to a tensor of bidegree $(0,0)$ (because it is a morphism of Hodge structures) and therefore is fixed by $G$ :

$$
\psi\left(g_{1} v, g_{1} v^{\prime}\right)=g_{2}^{n} \psi\left(v, v^{\prime}\right), \text { all }\left(g_{1}, g_{2}\right) \in G(\mathbb{Q}) \subset \mathrm{GL}(V) \times \mathbb{Q}^{\times}, \quad\left(v, v^{\prime}\right) \in V .
$$

Recall that if $H$ is a real algebraic group and $\sigma$ is an involution of $H_{\mathbb{C}}$, then the real-form of $H$ defined by $\sigma$ is a real algebraic group $H_{\sigma}$ endowed with an isomorphism $H_{\mathbb{C}} \rightarrow\left(H_{\sigma}\right)_{\mathbb{C}}$ under which complex conjugation on $H_{\sigma}(\mathbb{C})$ corresponds to $\sigma \circ$ (complex conjugation) on $H(\mathbb{C})$. We are going to use the following criterion: a connected algebraic group $H$ over $\mathbb{R}$ is reductive if it has a compact real-form $H_{\sigma}$. To prove the criterion, it suffices to show that $H_{\sigma}$ is reductive. On any finite-dimensional representation $V$ of $H$, there is an $H_{\sigma}$-invariant positive definite symmetric form, namely,

$$
\langle u, v\rangle_{0}=\int_{H_{\sigma}}\langle h u, h v\rangle d h,
$$

where $\langle$,$\rangle is any positive definite symmetric form on V$. If $W$ is an $H_{\sigma}$-stable subspace of $V$, then its orthogonal complement is also $H_{\sigma}$-stable. Thus every finite-dimensional representation of $H_{\sigma}$ is semisimple, and this implies that $H_{\sigma}$ is reductive (Deligne and Milne $1982, \S 2)$.

We shall apply the criterion to the special Mumford-Tate group of $(V, h)$,

$$
G^{0} \stackrel{\text { def }}{=} \operatorname{Ker}\left(G \rightarrow \mathbb{G}_{m}\right)
$$

Let $G^{1}$ be the smallest $\mathbb{Q}$-rational subgroup of $\operatorname{GL}(V) \times \mathbb{G}_{m}$ such that $G_{\mathbb{R}}^{1}$ contains $h\left(U^{1}\right)$, where $U^{1}(\mathbb{R})=\left\{z \in \mathbb{C}^{\times} \mid z \bar{z}=1\right\}$. Then $G^{1} \subset G$, and in fact $G^{1} \subset G^{0}$. Since $G_{\mathbb{R}}^{1}$. $h\left(\mathbb{C}^{\times}\right)=G_{\mathbb{R}}$ and $h\left(U^{1}\right)=\operatorname{Ker}\left(h\left(\mathbb{C}^{\times}\right) \rightarrow \mathbb{G}_{m}\right)$, it follows that $G^{0}=G^{1}$, and therefore $G^{0}$ is connected.

Since $C=h(i)$ acts as 1 on $\mathbb{Q}(1), C \in G^{0}(\mathbb{R})$. Its square $C^{2}$ acts as $(-1)^{n}$ on $V$ and therefore lies in the centre of $G^{0}(\mathbb{R})$. The inner automorphism adC of $G_{\mathbb{R}}$ defined by $C$ is therefore an involution. For $u, v \in V_{\mathbb{C}}$, and $g \in G^{0}(\mathbb{C})$, we have

$$
\psi(u, C \bar{v})=\psi(g u, g C \bar{v})=\psi\left(g u, C C^{-1} g C \bar{v}\right)=\psi\left(g u, C \overline{g^{*} v}\right)
$$

where $g^{*}=C^{-1} \bar{g} C=(\operatorname{ad} C)(\bar{g})$. Thus, the positive definite form $\phi(u, v) \stackrel{\text { def }}{=} \psi(u, C v)$ on $V_{\mathbb{R}}$ is invariant under the real-form of $G^{0}$ defined by $\operatorname{ad} C$, and so this real-form is compact.

EXAMPLE 3.7. (Abelian varieties of CM-type). A $\boldsymbol{C M}$-field is a quadratic totally imaginary extension of a totally real field, and a CM-algebra is a finite product of CM-fields. Let $E$ be a CM-algebra, and let $\iota_{E}$ be the involution of $E$ such that $\iota \circ \sigma=\sigma \circ \iota_{E}$ for all $\sigma: E \rightarrow \mathbb{C}$. Let $I$

$$
S=\operatorname{Hom}(E, \overline{\mathbb{Q}})=\operatorname{Hom}(E, \mathbb{C})==\operatorname{spec}\left(E_{\mathbb{C}}\right) .
$$

A CM-type for $E$ is a subset $\Sigma \subset S$ such that

$$
S=\Sigma \sqcup \iota \Sigma \quad \text { (disjoint union) }
$$


To the pair $(E, \Sigma)$, there is attached an abelian variety $A$ with $A(\mathbb{C})=\mathbb{C}^{\Sigma} / \Sigma\left(\mathcal{O}_{E}\right)$ where $\mathcal{O}_{E}$, the ring of integers in $E$, is embedded in $\mathbb{C}^{\Sigma}$ by the map $u \mapsto(\sigma u)_{\sigma \in \Sigma}$. Obviously, $E$ acts on $A$. Moreover, from the choice of a nonzero element of $H_{1}(A, \mathbb{Q})$, we obtain an isomorphism $H_{1}(A, \mathbb{Q}) \simeq E$, and

$$
\begin{aligned}
H_{1}(A) \otimes \mathbb{C} \simeq E \otimes_{\mathbb{Q}} \mathbb{C} & \stackrel{\simeq}{\rightarrow} \mathbb{C}^{S}=\mathbb{C}^{\Sigma} \oplus \mathbb{C}^{\iota \Sigma} \\
u \otimes 1 & \mapsto(\sigma u)_{\sigma \in S}
\end{aligned}
$$

with $\mathbb{C}^{\Sigma}$ the $(-1,0)$-component of $H_{1}(A) \otimes \mathbb{C}$ and $\mathbb{C}^{\iota \Sigma}$ the $(0,-1)$-component. Thus, $\mu(z)$ acts as $z$ on $\mathbb{C}^{\Sigma}$ and as 1 on $\mathbb{C}^{l \Sigma}$.

Let $G$ be the Mumford-Tate group of $H_{1}(A)$. The actions of $\mu\left(\mathbb{C}^{\times}\right)$and $E^{\times}$on $H_{1}(A) \otimes$ $\mathbb{C}$ commute. As $E^{\times}$is its own commutant in $\operatorname{GL}\left(H_{1}(A)\right)$, this implies that $\mu\left(\mathbb{C}^{\times}\right) \subset$ $(E \otimes \mathbb{C})^{\times}$and $G$ is the smallest algebraic subgroup of $E^{\times} \times \mathbb{Q}^{\times}$such that $G(\mathbb{C})$ contains $\mu\left(\mathbb{C}^{\times}\right)$. In particular, $G$ is a torus, and can be described by its cocharacter group $Y(G) \stackrel{\text { def }}{=}$ $\operatorname{Hom}_{\overline{\mathbb{Q}}}\left(\mathbb{G}_{m}, G\right)$.

Clearly,

$$
Y(G) \subset Y\left(E^{\times}\right) \times Y\left(\mathbb{G}_{m}\right)=\mathbb{Z}^{S} \times \mathbb{Z} .
$$

Note that $\mu \in Y(G)$ is equal to $\sum_{s \in \Sigma} e_{s}+e_{0}$, where $\left(e_{S}\right)_{s \in S} \subset \mathbb{Z}^{S}$ is the basis dual to the basis $S$ of $X\left(E^{\times}\right)$and $e_{0}$ is the element 1 of the last copy of $\mathbb{Z}$. The following are obvious:

(a) $\left(\mathbb{Z}^{S} \times \mathbb{Z}\right) / Y(G)$ is torsion-free;

(b) $\mu \in Y(G)$;

(c) $Y(G)$ is stable under $\operatorname{Gal}(\overline{\mathbb{Q}} / \mathbb{Q})$; thus $Y(G)$ is the $\operatorname{Gal}(\overline{\mathbb{Q}} / \mathbb{Q})$-module generated by $\mu$;

(d) since $\mu+\iota \mu=1$ on $S$,

$$
Y(G) \subset\left\{\sum n_{s} e_{s}+n_{0} e_{0} \in \mathbb{Z}^{S} \times \mathbb{Z} \mid n_{s}+n_{\iota s}=\text { constant }\right\} .
$$

Let $F$ be the subalgebra of $E$ whose elements are fixed by $\iota_{E}$ (thus, $F$ is a product of totally real fields). Then (d) says that

$$
G(\mathbb{Q}) \subset\left\{(x, y) \in E^{\times} \times \mathbb{Q}^{\times} \mid \mathrm{Nm}_{E / F}(x) \in \mathbb{Q}^{\times}\right\} .
$$

\section{Principle A}

THEOREM 3.8 (PRINCIPLE A). Let $\left(X_{\alpha}\right)_{\alpha}$ be a family of varieties over $\mathbb{C}$, and consider spaces $T$ obtained by tensoring spaces of the form $H_{\mathrm{B}}^{n_{\alpha}}\left(X_{\alpha}\right), H_{\mathrm{B}}^{n_{\alpha}}\left(X_{\alpha}\right)^{\vee}$, and $\mathbb{Q}(1)$. Let $t_{i} \in T_{i}, i=1, \ldots, N$ ( $T_{i}$ of the above type) be absolute Hodge cycles, and let $G$ be the subgroup of

$$
\prod_{\alpha, n_{\alpha}} \operatorname{GL}\left(H_{\mathrm{B}}^{n_{\alpha}}\left(X_{\alpha}\right)\right) \times \mathbb{G}_{m}
$$

fixing the $t_{i}$. If $t$ belongs to some $T$ and is fixed by $G$, then it is an absolute Hodge cycle.

We first need a lemma.

LeMma 3.9. Let $G$ be an algebraic group over $\mathbb{Q}$, and let $P$ be a $G$-torsor of isomorphisms $H_{\sigma}^{\alpha} \rightarrow H_{\tau}^{\alpha}$ where $\left(H_{\sigma}^{\alpha}\right)_{\alpha}$ and $\left(H_{\tau}^{\alpha}\right)_{\alpha}$ are families of $\mathbb{Q}$-rational representations of $G$. Let $T_{\sigma}$ and $T_{\tau}$ be like spaces of tensors constructed out of $H_{\sigma}$ and $H_{\tau}$ respectively. Then $P$ defines a map $T_{\sigma}^{G} \rightarrow T_{\tau}$. 
ProOF. Locally for the étale topology on $\operatorname{Spec}(\mathbb{Q})$, points of $P$ define isomorphisms $T_{\sigma} \rightarrow T_{\tau}$. The restriction to $T_{\sigma}^{G}$ of such a map is independent of the point. Thus, by étale descent theory, they define a map of vector spaces $T_{\sigma}^{G} \rightarrow T_{\tau}$.

Proof OF THEOREM 3.8. We remove the identification of the ground field with $\mathbb{C}$. Thus, the ground field is now a field $k$ equipped with an isomorphism $\sigma: k \rightarrow \mathbb{C}$. Let $\tau: k \rightarrow \mathbb{C}$ be a second isomorphism. We can assume that $t$ and the $t_{i}$ all belong to the same space $T$. The canonical inclusions of cohomology groups

$$
H_{\sigma}\left(X_{\alpha}\right) \hookrightarrow H_{\sigma}\left(X_{\alpha}\right) \otimes\left(\mathbb{C} \times \mathbb{A}_{f}\right) \hookleftarrow H_{\tau}\left(X_{\alpha}\right)
$$

induce maps

$$
T_{\sigma} \hookrightarrow T \otimes\left(\mathbb{C} \times \mathbb{A}_{f}\right) \hookleftarrow T_{\tau} .
$$

We shall regard these maps as inclusions. Thus,

$$
\begin{aligned}
\left(t_{1}, \ldots, t_{N}, t\right) & \subset T_{\sigma} \subset T \otimes\left(\mathbb{C} \times \mathbb{A}_{f}\right), \\
\left(t_{1}, \ldots, t_{N}\right) & \subset T_{\tau} \subset T \otimes\left(\mathbb{C} \times \mathbb{A}_{f}\right) .
\end{aligned}
$$

Let $P$ be the functor of $\mathbb{Q}$-algebras such that

$$
P(R)=\left\{p: H_{\sigma} \otimes R \stackrel{\approx}{\rightarrow} H_{\tau} \otimes R \mid p \text { maps } t_{i}\left(\text { in } T_{\sigma} \text { ) to } t_{i} \text { (in } T_{\tau} \text { ), } i=1, \ldots, N\right\} .\right.
$$

The existence of the canonical inclusions mentioned above shows that $P\left(\mathbb{C} \times \mathbb{A}_{f}\right)$ is nonempty, and it is easily seen that $P$ is a $G$-torsor.

On applying the lemma (and its proof) in the above situation, we obtain a map $T_{\sigma}^{G} \rightarrow T_{\tau}$ such that

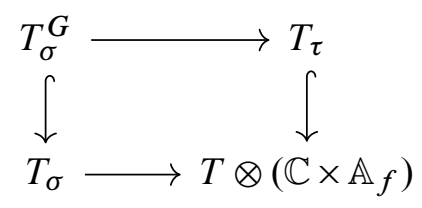

commutes. This means that $T_{\sigma}^{G} \subset T_{\tau}$, and therefore $t \in T_{\tau}$.

It remains to show that the component $t_{\mathrm{dR}}$ of $t$ in $T \otimes \mathbb{C}=T_{\mathrm{dR}}$ lies in $F^{0} T_{\mathrm{dR}}$. But for a rational $s \in T_{\mathrm{dR}}$,

$$
s \in F^{0} T_{\mathrm{dR}} \Longleftrightarrow s \text { is fixed by } \mu\left(\mathbb{C}^{\times}\right) .
$$

Thus, $\left(t_{i}\right)_{\mathrm{dR}} \in F^{0}, i=1,2, \ldots, N$, implies $G \supset \mu\left(\mathbb{C}^{\times}\right)$, which implies that $t_{\mathrm{dR}} \in F^{0}$. 


\section{Construction of some absolute Hodge cycles}

\section{Hermitian forms}

Recall that a number field $E$ is a CM-field if, for each embedding $E \hookrightarrow \mathbb{C}$, complex conjugation induces a nontrivial automorphism $e \mapsto \bar{e}$ on $E$ that is independent of the embedding. The fixed field of the automorphism is then a totally real field $F$ over which $E$ has degree two.

A bi-additive form

$$
\phi: V \times V \rightarrow E
$$

on a vector space $V$ over a CM-field $E$ is Hermitian if

$$
\phi(e v, w)=e \phi(v, w), \quad \phi(v, w)=\overline{\phi(w, v)}, \quad \text { all } e \in E, v, w \in V .
$$

For any embedding $\tau: F \hookrightarrow \mathbb{R}$ we obtain a Hermitian form $\phi_{\tau}$ in the usual sense on the vector space $V_{\tau}=V \otimes_{F, \tau} \mathbb{R}$, and we let $a_{\tau}$ and $b_{\tau}$ denote the dimensions of the maximal subspaces of $V_{\tau}$ on which $\phi_{\tau}$ is positive definite and negative definite respectively. If $d=\operatorname{dim} V$, then $\phi$ defines a Hermitian form on $\bigwedge^{d} V$ that, relative to some basis vector, is of the form $(x, y) \mapsto f x \bar{y}$. The element $f$ is in $F$, and is independent of the choice of the basis vector up to multiplication by an element of $\mathrm{Nm}_{E / F} E^{\times}$. It is called the discriminant of $\phi$. Let $\left(v_{1}, \ldots, v_{d}\right)$ be an orthogonal basis for $\phi$, and let $\phi\left(v_{i}, v_{i}\right)=c_{i}$; then $a_{\tau}$ is the number of $i$ for which $\tau c_{i}>0, b_{\tau}$ the number of $i$ for which $\tau c_{i}<0$, and $f=\prod c_{i}\left(\bmod \mathrm{Nm}_{E / F} E^{\times}\right)$. If $\phi$ is nondegenerate, then $f \in F^{\times} / \mathrm{Nm} E^{\times}$, and

$$
a_{\tau}+b_{\tau}=d, \quad \operatorname{sign}(\tau f)=(-1)^{b_{\tau}}, \text { all } \tau .
$$

PROPOSITION 4.1. Suppose given nonnegative integers $\left(a_{\tau}, b_{\tau}\right)_{\tau: F \hookrightarrow \mathbb{C}}$ and an element $f \in F^{\times} / \mathrm{Nm} E^{\times}$satisfying (1). Then there exists a non-degenerate Hermitian form $\phi$ on an $E$-vector space $V$ of dimension $d$ with invariants $\left(a_{\tau}, b_{\tau}\right)$ and $f$; moreover, $(V, \phi)$ is unique up to isomorphism.

PROOF. The result is due to Landherr 1936. Today one prefers to regard it as a consequence of the Hasse principle for simply connected semisimple algebraic groups and the classification of Hermitian forms over local fields.

Corollary 4.2. Let $(V, \phi)$ be a non-degenerate Hermitian space, and let $d=\operatorname{dim} V$. The following conditions are equivalent:

(a) $a_{\tau}=b_{\tau}$ for all $\tau$ and $\operatorname{disc}(f)=(-1)^{d / 2}$;

(b) there exists a totally isotropic subspace of $V$ of dimension $d / 2$.

Proof. Let $W$ be a totally isotropic subspace of $V$ of dimension $d / 2$. The map $v \mapsto$ $\phi(-, v): V \rightarrow W^{\vee}$ induces an antilinear isomorphism $V / W \rightarrow W^{\vee}$. Thus, a basis $e_{1}, \ldots, e_{d / 2}$ of $W$ can be extended to a basis $\left\{e_{i}\right\}$ of $V$ such that

$$
\begin{aligned}
& \phi\left(e_{i}, e_{\frac{d}{2}+i}\right)=1, \quad 1 \leq i \leq d / 2, \\
& \phi\left(e_{i}, e_{j}\right)=0, \quad j \neq i \pm d / 2 .
\end{aligned}
$$


It is now easy to check that $(V, \phi)$ satisfies (a). Conversely, $\left(E^{d}, \phi\right)$ where

$$
\phi\left(\left(a_{i}\right),\left(b_{i}\right)\right)=\sum_{1 \leq i \leq d / 2} a_{i} \bar{b}_{\frac{d}{2}+i}+a_{\frac{d}{2}+i} \bar{b}_{i}
$$

is, up to isomorphism, the only Hermitian space satisfying (a), and it also satisfies (b).

A Hermitian form satisfying the equivalent conditions of the corollary will be said to be split (because then the algebraic group $\operatorname{Aut}_{E}(V, \phi)$ over $F$ is split).

We shall need the following lemma from linear algebra.

LEMma 4.3. Let $k$ be a field, and let $V$ be a free finitely generated module over an étale $k$-algebra $k^{\prime}$ (i.e., $k^{\prime}$ is a finite product of finite separable field extensions of $k$ ).

(a) The map

$$
f \mapsto \operatorname{Tr}_{k^{\prime} / k} \circ f: \operatorname{Hom}_{k^{\prime}}\left(V, k^{\prime}\right) \rightarrow \operatorname{Hom}_{k}(V, k)
$$

is an isomorphism of $k$-vector spaces.

(b) $\bigwedge_{k^{\prime}}^{n} V$ is, in a natural way, a direct summand of $\bigwedge_{k}^{n} V$.

PROOF. (a) As the pairing $\operatorname{Tr}_{k^{\prime} / k}: k^{\prime} \times k^{\prime} \rightarrow k$ is nondegenerate, the map $f \mapsto \operatorname{Tr}_{k^{\prime} / k} \circ f$ is injective, and it is onto because the two spaces have the same dimension over $k$.

(b) There are obvious maps

$$
\begin{aligned}
\bigwedge_{k}^{n} V & \rightarrow \bigwedge_{k^{\prime}}^{n} V \\
\bigwedge_{k}^{n} V^{\vee} & \rightarrow \bigwedge_{k^{\prime}}^{n} V^{\vee}
\end{aligned}
$$

where $V^{\vee}=\operatorname{Hom}_{k^{\prime}}\left(V, k^{\prime}\right) \simeq \operatorname{Hom}_{k}(V, k)$. The pairing

$$
\bigwedge^{n} V^{\vee} \times \bigwedge^{n} V \rightarrow k
$$

determined by

$$
\left(f_{1} \wedge \cdots \wedge f_{n}, v_{1} \otimes \cdots \otimes v_{n}\right)=\operatorname{det}\left(\left\langle f_{i} \mid v_{j}\right\rangle\right)
$$

induces an isomorphism $\left(\bigwedge^{n} V^{\vee}\right) \simeq\left(\bigwedge^{n} V\right)^{\vee}$ (Bourbaki, N., Algébre Multilinéaire, Hermann, 1958, §8), and so the second map gives rise to a map $\bigwedge_{k^{\prime}}^{n} V \rightarrow \bigwedge_{k}^{n} V$, which is left inverse to the first.

Alternatively, and more elegantly, descent theory shows that it suffices to prove the proposition with $k^{\prime}=k^{S}, S=\operatorname{Hom}_{k}\left(k^{\prime}, \bar{k}\right)$. Then $V=\bigoplus_{s \in S} V_{s}$ with the $V_{s}$ subspaces of the same dimension, and the map in (a) becomes $f=\left(f_{s}\right) \mapsto \sum f_{s}$, which is obviously an isomorphism. For (b), note that ${ }^{5}$

$$
\bigwedge_{k}^{n} V=\bigoplus_{\sum n_{s}=n}\left(\bigotimes_{s \in S} \bigwedge_{k}^{n_{s}} V_{s}\right) \supset \bigoplus_{s \in S} \bigwedge_{k}^{n} V_{s}=\bigwedge_{k^{\prime}}^{n} V
$$

The first sum is over the set of families $\left(n_{s}\right)_{s \in S}$ such that $n_{s} \in \mathbb{N}$ and $\sum_{s \in S} n_{s}=n$, while the second is over the set of such families with $n_{s}=n$ for some $s$.

\footnotetext{
${ }^{5}$ Because $V$ is a free $k^{S}$-module,

$$
V=W \otimes_{k} k^{S}=\bigoplus_{s \in S} W \otimes_{k} k=\bigoplus_{s \in S} V_{s}
$$

for some $k$-vector space $W$. Correspondingly,

$$
\bigwedge_{k}^{n} V=\left(\bigwedge_{k}^{n} W\right) \otimes_{k} k^{S}=\bigoplus_{s \in S}\left(\bigwedge_{k}^{n} W\right) \otimes_{k} k=\bigoplus_{s \in S} \bigwedge_{k}^{n} V_{s}
$$
}




\section{Conditions for $\bigwedge_{E}^{d} H^{1}(A, \mathbb{Q})$ to consist of absolute Hodge cycles}

Let $A$ be an abelian variety over $\mathbb{C}$ and let $v: E \rightarrow \operatorname{End}(A)$ be a homomorphism with $E$ a CMfield (in particular, this means that $\left.v(1)=\mathrm{id}_{A}\right)$. Let $d$ be the dimension of $H_{1}(A, \mathbb{Q})$ over $E$, so that $d[E: \mathbb{Q}]=2 \operatorname{dim} A$. When $H_{1}(A, \mathbb{R})$ is identified with the tangent space to $A$ at zero, it acquires a complex structure; we denote by $J$ the $\mathbb{R}$-linear endomorphism "multiplication by $i$ " of $H_{1}(A, \mathbb{R})$. If $h: \mathbb{C}^{\times} \rightarrow \operatorname{GL}\left(H^{1}(A, \mathbb{R})\right)$ is the homomorphism determined by the Hodge structure on $H^{1}(A, \mathbb{R})$, then $h(i) \leftrightarrow J$ under the isomorphism $\operatorname{GL}\left(H^{1}(A, \mathbb{R})\right) \simeq$ $\mathrm{GL}\left(H_{1}(A, \mathbb{R})\right)$ determined by $H^{1}(A, \mathbb{R}) \simeq H_{1}(A, \mathbb{R})^{\vee}$.

Corresponding to the decomposition

$$
e \otimes z \mapsto(\ldots, \sigma e \cdot z, \ldots): E \otimes_{\mathbb{Q}} \mathbb{C} \stackrel{\simeq}{\rightarrow} \prod_{\sigma \in S} \mathbb{C}, \quad S=\operatorname{Hom}(E, \mathbb{C}),
$$

there is a decomposition

$$
H_{B}^{1}(A) \otimes \mathbb{C} \stackrel{\simeq}{\rightarrow} \bigoplus_{\sigma \in S} H_{B, \sigma}^{1} \quad(E \text {-linear isomorphism })
$$

such that $e \in E$ acts on the complex vector space $H_{B, \sigma}^{1}$ as $\sigma e$. Each $H_{B, \sigma}^{1}$ has dimension $d$, and (as $E$ respects the Hodge structure on $H_{B}^{1}(A)$ ) acquires a Hodge structure

$$
H_{B, \sigma}^{1}=H_{B, \sigma}^{1,0} \oplus H_{B, \sigma}^{0,1} \text {. }
$$

Let $a_{\sigma}=\operatorname{dim} H_{B, \sigma}^{1,0}$ and $b_{\sigma}=\operatorname{dim} H_{B, \sigma}^{0,1}$; thus $a_{\sigma}+b_{\sigma}=d$.

Proposition 4.4. The subspace $\bigwedge_{E}^{d} H_{\mathrm{B}}^{1}(A)$ of $H^{d}(A, \mathbb{Q})$ is purely of bidegree $\left(\frac{d}{2}, \frac{d}{2}\right)$ if $\mathrm{M} .10$ and only if $a_{\sigma}=\frac{d}{2}=b_{\sigma}$ for all $\sigma \in S$.

Proof. Note that $H^{d}(A, \mathbb{Q}) \simeq \bigwedge_{\mathbb{Q}}^{d} H^{1}(A, \mathbb{Q})$, and so (4.3) canonically identifies $\bigwedge_{E}^{d} H_{\mathrm{B}}^{1}(A)$ with a subspace of $H_{\mathrm{B}}^{d}(A)$. As in the last line of the proof of (4.3), we have

$$
\begin{aligned}
\left(\bigwedge_{E}^{d} H_{\mathrm{B}}^{1}\right) \otimes \mathbb{C} & \simeq \bigwedge_{E \otimes \mathbb{C}}^{d}\left(H_{\mathrm{B}}^{1} \otimes \mathbb{C}\right) \\
& \simeq \bigoplus_{\sigma \in S} \wedge^{d} H_{\mathrm{B}, \sigma}^{1} \\
& \simeq \bigoplus_{\sigma \in S} \wedge^{d}\left(H_{\mathrm{B}, \sigma}^{1,0} \oplus H_{\mathrm{B}, \sigma}^{0,1}\right) \\
& \simeq \bigoplus_{\sigma \in S} \wedge^{a_{\sigma}} H_{\mathrm{B}, \sigma}^{1,0} \otimes \wedge^{b_{\sigma}} H_{\mathrm{B}, \sigma}^{0,1},
\end{aligned}
$$

and $\bigwedge^{a_{\sigma}} H_{\mathrm{B}, \sigma}^{1,0}$ and $\bigwedge^{b_{\sigma}} H_{\mathrm{B}, \sigma}^{0,1}$ are purely of bidegree $\left(a_{\sigma}, 0\right)$ and $\left(0, b_{\sigma}\right)$ respectively.

Thus, in this case, $\left(\bigwedge_{E}^{d} H_{\mathrm{B}}^{1}(A)\right)\left(\frac{d}{2}\right)$ consists of Hodge cycles, and we would like to show that it consists of absolute Hodge cycles. In one special case, this is easy.

LEMMA 4.5. Let $A_{0}$ be an abelian variety of dimension $\frac{d}{2}$ and let $A=A_{0} \otimes_{\mathbb{Q}} E$. Then the subspace $\bigwedge_{E}^{d} H^{1}(A, \mathbb{Q})\left(\frac{d}{2}\right)$ of $H^{d}(A, \mathbb{Q})\left(\frac{d}{2}\right)$ consists of absolute Hodge cycles. 
PROOF. There is a commutative diagram

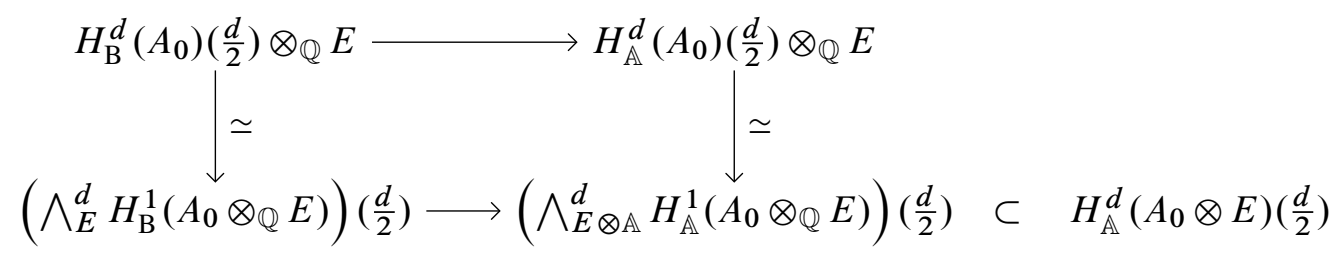

in which the vertical maps are induced by $H^{1}\left(A_{0}\right) \otimes E \stackrel{\simeq}{\rightarrow} H^{1}\left(A_{0} \otimes E\right)$. From this, and similar diagrams corresponding to isomorphisms $\sigma: \mathbb{C} \rightarrow \mathbb{C}$, one sees that

$$
H_{\mathbb{A}}^{d}\left(A_{0}\right)\left(\frac{d}{2}\right) \otimes E \hookrightarrow H_{\mathbb{A}}^{d}\left(A_{0} \otimes E\right)\left(\frac{d}{2}\right)
$$

induces an inclusion

$$
C_{\mathrm{AH}}^{d}\left(A_{0}\right) \otimes E \hookrightarrow C_{\mathrm{AH}}^{d}\left(A_{0} \otimes E\right) .
$$

But $C_{\mathrm{AH}}^{d}\left(A_{0}\right)=H_{\mathrm{B}}^{d}\left(A_{0}\right)\left(\frac{d}{2}\right)$ since $H_{\mathrm{B}}^{d}\left(A_{0}\right)\left(\frac{d}{2}\right)$ is a one-dimensional space generated by the class of any point on $A_{0}$. This completes the proof as the above diagram shows that the image of $H_{\mathrm{B}}^{d}\left(A_{0}\right)\left(\frac{d}{2}\right)$ in $H_{\mathrm{B}}^{d}(A)\left(\frac{d}{2}\right)$ is $\bigwedge_{E}^{d} H_{\mathrm{B}}^{1}(A)\left(\frac{d}{2}\right)$.

In order to prove the general result, we need to consider families of abelian varieties (ultimately, we wish to apply (2.15)), and for this we need to consider polarized abelian varieties. A polarization $\theta$ on $A$ is determined by a Riemann form, i.e., a $\mathbb{Q}$-bilinear alternating form $\psi$ on $H_{1}(A, \mathbb{Q})$ such that the form $(z, w) \mapsto \psi(z, J w)$ on $H_{1}(A, \mathbb{R})$ is symmetric and definite; two Riemann forms $\psi$ and $\psi^{\prime}$ on $H_{1}(A, \mathbb{Q})$ correspond to the same polarization if and only if there is an $a \in \mathbb{Q}^{\times}$such that $\psi^{\prime}=a \psi$. We shall consider only triples $(A, \theta, v)$ in which the Rosati involution defined by $\theta$ induces complex conjugation on $E$. (The Rosati involution $e \mapsto^{t} e: \operatorname{End}(A) \rightarrow \operatorname{End}(A)$ is determined by the condition

$$
\left.\psi(e v, w)=\psi\left(v,{ }^{t} e w\right), \quad v, w \in H_{1}(A, \mathbb{Q}) .\right)
$$

LEMmA 4.6. Let $f \in E^{\times}$be such that $\bar{f}=-f$, and let $\psi$ be a Riemann form for $A$. There exists a unique $E$-Hermitian form $\phi$ on $H_{1}(A, \mathbb{Q})$ such that $\psi(x, y)=\operatorname{Tr}_{E / \mathbb{Q}}(f \phi(x, y))$.

We first need:

Sublemma 4.7. Let $V$ and $W$ be finite-dimensional vector spaces over $E$, and let $\psi: V \times$ $W \rightarrow \mathbb{Q}$ be a $\mathbb{Q}$-bilinear form such that $\psi(e v, w)=\psi(v, e w)$ for $e \in E$. Then there exists a unique $E$-bilinear form $\phi$ such that $\psi(v, w)=\operatorname{Tr}_{E / \mathbb{Q}} \phi(v, w)$.

Proof. The condition says that $\psi$ defines a $\mathbb{Q}$-linear map $V \otimes_{E} W \rightarrow \mathbb{Q}$. Let $\phi$ be the element of $\operatorname{Hom}_{\mathbb{Q}}\left(V \otimes_{E} W, E\right)$ corresponding to $\psi$ under the isomorphism (see 4.3(a))

$$
\operatorname{Hom}_{E}\left(V \otimes_{E} W, E\right) \simeq \operatorname{Hom}_{\mathbb{Q}}\left(V \otimes_{E} W, \mathbb{Q}\right) .
$$

Proof OF LEMMA 4.6. We apply (4.7) with $V=H_{1}(A, \mathbb{Q})=W$, but with with $E$ acting through complex conjugation on $W$. This gives a sesquilinear $\phi_{1}$ such that $\psi(x, y)=$ $\operatorname{Tr}_{E / \mathbb{Q}} \phi_{1}(x, y)$. Let $\phi=f^{-1} \phi_{1}$, so that $\psi(x, y)=\operatorname{Tr}_{E / \mathbb{Q}}(f \phi(x, y))$. Since $\phi$ is sesquilinear it remains to show that $\phi(x, y)=\overline{\phi(x, y)}$. As $\psi(x, y)=-\psi(y, x)$ for all $x, y \in$ $H_{1}(A, \mathbb{Q})$,

$$
\operatorname{Tr}(f \phi(x, y))=-\operatorname{Tr}(f \phi(y, x))=\operatorname{Tr}(\bar{f} \phi(y, x)) .
$$


On replacing $x$ by $e x$ with $e \in E$, we find that

$$
\operatorname{Tr}(f e \phi(x, y))=\operatorname{Tr}(\overline{f e} \phi(y, x)) .
$$

On the other hand,

$$
\operatorname{Tr}(f e \phi(x, y))=\operatorname{Tr}(\overline{f e \phi(x, y)})
$$

and so

$$
\operatorname{Tr}(\overline{f e} \phi(y, x))=\operatorname{Tr}(\overline{f e \phi(x, y)}) .
$$

As $\overline{f e}$ is an arbitrary element of $E$, the non-degeneracy of the trace implies that $\overline{\phi(x, y)}=$ $\phi(y, x)$. Finally, the uniqueness of $\phi$ is obvious from (4.7).

THEOREM 4.8. Let $A$ be an abelian variety over $\mathbb{C}$, and let $v: E \rightarrow \operatorname{End}(A)$ be a homomorphism with $E$ a $C M$-field. Let $d=\operatorname{dim}_{E} H^{1}(A, \mathbb{Q})$. Assume there exists a polarization $\theta$ for $A$ such that

(a) the Rosati involution of $\theta$ induces complex conjugation on $E$;

(b) there exists a split $E$-Hermitian form $\phi$ on $H_{1}(A, \mathbb{Q})$ and an $f \in E^{\times}$with $\bar{f}=-f$ such that $\psi(x, y) \stackrel{\text { def }}{=} \operatorname{Tr}_{E / \mathbb{Q}}(f \phi(x, y))$ is a Riemann form for $\theta$.

Then the subspace $\left(\bigwedge_{E}^{d} H^{1}(A, \mathbb{Q})\right)\left(\frac{d}{2}\right)$ of $H^{d}(A, \mathbb{Q})\left(\frac{d}{2}\right)$ consists of absolute Hodge cycles.

Proof. In the course of the proof, we shall see that (b) implies that $A$ satisfies the equivalent statements of (4.4). Thus, the theorem will follow from (2.15) and (4.5) once we have shown that there exists a connected smooth variety $S$ over $\mathbb{C}$ and an abelian scheme $Y$ over $S$ together with an action $v$ of $E$ on $Y / S$ such that ${ }^{6}$

(a) for all $s \in S,\left(Y_{S}, v_{s}\right)$ satisfies the equivalent conditions in (4.4);

(b) for some $s_{0} \in S, Y_{s_{0}}$ is isomorphic to $A_{0} \otimes_{\mathbb{Q}} E$, some $A_{0}$, with $e \in E$ acting as id $\otimes e$;

(c) for some $s_{1} \in S,\left(Y_{s_{1}}, v_{s_{1}}\right)=(A, v)$.

We shall first construct an analytic family of abelian varieties satisfying these conditions, and then pass to the quotient by a discrete group to obtain an algebraic family.

Let $H=H_{1}(A, \mathbb{Q})$ regarded as an $E$-space and chose $\theta, \psi, f$, and $\psi$ as in the statement of the theorem. We choose $i=\sqrt{-1}$ so that $\psi(x, h(i) y)$ is positive definite.

Consider the set of all quadruples $\left(A_{1}, \theta_{1}, v_{1}, k_{1}\right)$ in which $A_{1}$ is an abelian variety over $\mathbb{C}, v_{1}$ is an action of $E$ on $A_{1}, \theta_{1}$ is a polarization of $A$, and $k_{1}$ is an $E$-linear isomorphism $H_{1}\left(A_{1}, \mathbb{Q}\right) \rightarrow H$ carrying a Riemann form for $\theta_{1}$ into $c \psi$ for some $c \in \mathbb{Q}^{\times}$. From such a quadruple, we obtain a complex structure on $H(\mathbb{R})$ (corresponding via $k_{1}$ to the complex structure on $\left.H_{1}\left(A_{1}, \mathbb{R}\right)=\operatorname{Lie}\left(A_{1}\right)\right)$ such that:

(a) the action of $E$ commutes with the complex structure;

(b) $\psi$ is a Riemann form relative to the complex structure.

Conversely, a complex structure on $H \otimes \mathbb{R}$ satisfying (a) and (b) determines a quadruple $\left(A_{1}, \theta_{1}, v_{1}, k_{1}\right)$ with $H_{1}\left(A_{1}, \mathbb{Q}\right)=H\left(\right.$ as an $E$-module), $\operatorname{Lie}\left(A_{1}\right)=H \otimes \mathbb{R}$ (endowed with the given complex structure), $\theta_{1}$ the polarization with Riemann form $\psi$, and $k_{1}$ the identity map. Moreover, two quadruples $\left(A_{1}, \theta_{1}, v_{1}, k_{1}\right)$ and $\left(A_{2}, \theta_{2}, v_{2}, k_{2}\right)$ are isomorphic if and only if they define the same complex structure on $H$. Let $X$ be the set of complex structures on $H$ satisfying (a) and (b). Our first task is to turn $X$ into an analytic manifold in such a way that the family of abelian varieties that it parametrizes becomes an analytic family.

\footnotetext{
${ }^{6}$ Added: we also need that there exists a locally constant subsytem of Hodge cycles.
} 
A point of $X$ is determined by an $\mathbb{R}$-linear map $J: H \otimes \mathbb{R} \rightarrow H \otimes \mathbb{R}, J^{2}=-1$, such that

(a') $J$ is $E$-linear, and

$\left(\mathrm{b}^{\prime}\right) \psi(x, J y)$ is symmetric and definite.

Note that $\psi(x, J y)$ is symmetric if and only if $\psi(J x, J y)=\psi(x, y)$. Let $F$ be the real subfield of $E$, and fix an isomorphism

$$
E \otimes_{\mathbb{Q}} \mathbb{R} \rightarrow \bigoplus_{\tau \in T} \mathbb{C}, \quad T=\operatorname{Hom}(F, \mathbb{R})
$$

such that $(f \otimes 1) \mapsto\left(i f_{\tau}\right)$ with $f_{\tau} \in \mathbb{R}, f_{\tau}>0$. Corresponding to this isomorphism, there is a decomposition

$$
H \otimes \mathbb{Q} \mathbb{R} \simeq \bigoplus_{\tau \in T} H_{\tau}
$$

in which each $H_{\tau}$ is a complex vector space. Condition ( $\left.\mathrm{a}^{\prime}\right)$ implies that $J=\oplus J_{\tau}$, where $J_{\tau}$ is a $\mathbb{C}$-linear isomorphism $H_{\tau} \rightarrow H_{\tau}$ such that $J_{\tau}^{2}=-1$. Let

$$
H_{\tau}=H_{\tau}^{+} \oplus H_{\tau}^{-} \text {, }
$$

where $H_{\tau}^{+}$and $H_{\tau}^{-}$are the eigenspaces of $J_{\tau}$ with eigenvalues $+i$ and $-i$ respectively. The compatibility of $\psi$ and $v$ implies

$$
(H, \psi) \otimes \mathbb{R} \simeq \bigoplus_{\tau \in T}\left(H_{\tau}, \psi_{\tau}\right)
$$

with $\psi_{\tau}$ an $\mathbb{R}$-bilinear alternating form on $H_{\tau}$ such that

$$
\psi_{\tau}(z x, y)=\psi_{\tau}(x, \bar{z} y), \quad z \in \mathbb{C} .
$$

The condition

$$
\psi(J x, J y)=\psi(x, y)
$$

implies that $H_{\tau}^{+}$is the orthogonal complement of $H_{\tau}^{-}$relative to $\psi_{\tau}$ :

$$
H_{\tau}=H_{\tau}^{+} \perp H_{\tau}^{-} .
$$

We also have

$$
(H, \phi) \otimes \mathbb{R} \simeq \bigoplus_{\tau \in T}\left(H_{\tau}, \phi_{\tau}\right)
$$

and $\psi_{\tau}(x, y)=\operatorname{Tr}_{\mathbb{C} / \mathbb{R}}\left(i f_{\tau} \phi_{\tau}(x, y)\right)$. As

$$
\psi(x, y)=\sum_{\tau} \operatorname{Tr}_{\mathbb{C} / \mathbb{R}}\left(i f_{\tau} \phi_{\tau}(x, y)\right),
$$

we find that

$$
\begin{aligned}
\psi(x, J x)>0 \text { all } x & \Longleftrightarrow \operatorname{Tr}_{\mathbb{C} / \mathbb{R}}\left(i f_{\tau} \phi_{\tau}\left(x_{\tau}, J x_{\tau}\right)\right)>0 \text { all } x_{\tau}, \tau \\
& \Longleftrightarrow \operatorname{Tr}_{\mathbb{C} / \mathbb{R}}\left(i \phi_{\tau}\left(x_{\tau}, J x_{\tau}\right)\right) \text { all } x_{\tau}, \tau, \\
& \Longleftrightarrow\left\{\begin{array}{l}
\phi_{\tau} \text { is positive definite on } H_{\tau}^{+}, \text {and } \\
\phi_{\tau} \text { is negative definite on } H_{\tau}^{-} .
\end{array}\right.
\end{aligned}
$$

This shows, in particular, that $H_{\tau}^{+}=H_{\tau}^{-1,0}$ and $H_{\tau}^{-}=H_{\tau}^{0,-1}$ each have dimension $d / 2$ (cf. 4.4). Let $X^{+}$and $X^{-}$be the sets of $J \in X$ for which $\psi(x, J y)$ is positive definite and 
negative definite respectively. Then $X$ is a disjoint union $X=X^{+} \sqcup X^{-}$. As $J$ is determined by its $+i$ eigenspace we see that $X^{+}$can be identified with

$$
\left\{\left(V_{\tau}\right)_{\tau \in T} \mid V_{\tau} \text { a maximal subspace of } H_{\tau} \text { such that } \phi_{\tau}>0 \text { on } V_{\tau}\right\} \text {. }
$$

This is an open connected complex submanifold of a product of Grassman manifolds

$$
X^{+} \subset \prod_{\tau \in T} \operatorname{Grass}_{d / 2}\left(V_{\tau}\right)
$$

Moreover, there is an analytic structure on $X^{+} \times V(\mathbb{R})$ such that $X^{+} \times V(\mathbb{R}) \rightarrow X^{+}$is analytic and the inverse image of $J \in X^{+}$is $V(\mathbb{R})$ with the complex structure provided by $J$. On dividing $V(\mathbb{R})$ by an $\mathcal{O}_{E}$-stable lattice $V(\mathbb{Z})$ in $V$, we obtain the sought analytic family $B$ of abelian varieties.

Note that $A$ is a member of the family. We shall next show that there is also an abelian variety of the form $A_{0} \otimes E$ in the family. To do this, we only have to show that there exists a quadruple $\left(A_{1}, \theta_{1}, v_{1}, k_{1}\right)$ of the type discussed above with $A_{1}=A_{0} \otimes E$. Let $A_{0}$ be any abelian variety of dimension $d / 2$ and define $v_{1}: E \rightarrow \operatorname{End}\left(A_{0} \otimes E\right)$ so that $e \in E$ acts on $H_{1}\left(A_{0} \otimes E\right)=H_{1}\left(A_{0}\right) \otimes E$ through its action on $E$. A Riemann form $\psi_{0}$ on $A_{0}$ extends in an obvious way to a Riemann form $\psi_{1}$ on $A_{1}$ that is compatible with the action of $E$. We define $\theta_{1}$ to be the corresponding polarization, and let $\phi_{1}$ be the Hermitian form on $H_{1}\left(A_{0} \otimes E, \mathbb{Q}\right)$ such that $\psi_{1}=\operatorname{Tr}_{E / \mathbb{Q}}\left(f \phi_{1}\right)$ (see 4.6). If $I_{0} \subset H_{1}\left(A_{0}, \mathbb{Q}\right)$ is a totally isotropic subspace of $H_{1}\left(A_{0}, \mathbb{Q}\right)$ of (maximum) dimension $d / 2$, then $I_{0} \otimes E$ is a totally isotropic subspace of dimension $d / 2$ over $E$, which (by 4.2) shows that the Hermitian space $\left(H_{1}\left(A_{0} \otimes E, \mathbb{Q}\right), \phi_{1}\right)$ is split. There is therefore an $E$-linear isomorphism $k_{1}:\left(H_{1}\left(A_{0} \otimes E, \mathbb{Q}\right), \phi_{1}\right) \rightarrow(H, \phi)$ which carries $\psi_{1}=\operatorname{Tr}_{E / \mathbb{Q}}\left(f \phi_{1}\right)$ to $\psi=\operatorname{Tr}_{E / \mathbb{Q}}\left(f \phi_{1}\right)$. This completes this part of the proof.

Let $n$ be an integer $\geq 3$, and let $\Gamma$ be the set of $\mathcal{O}_{E}$-isomorphisms $g: V(\mathbb{Z}) \rightarrow V(\mathbb{Z})$ preserving $\psi$ and such that $(g-1) V(\mathbb{Z}) \subset n V(\mathbb{Z})$. Then $\Gamma$ acts on $X^{+}$by $J \mapsto g \circ J \circ g^{-1}$ and (compatibly) on $B$. On forming the quotients, we obtain a map

$$
\Gamma \backslash B \rightarrow \Gamma \backslash X^{+}
$$

which is an algebraic family of abelian varieties. In fact, $\Gamma \backslash X^{+}$is the moduli variety for quadruples $\left(A_{1}, \theta_{1}, v_{1}, k_{1}\right)$ in which $A_{1}, \theta_{1}$, and $v_{1}$ are essentially as before, but now $k_{1}$ is a level $n$ structure

$$
k_{1}: A_{n}(\mathbb{C})=H_{1}(A, \mathbb{Z} / n \mathbb{Z}) \stackrel{\approx}{\rightarrow} V(\mathbb{Z}) / n V(\mathbb{Z}) ;
$$

the map $X^{+} \rightarrow \Gamma \backslash X^{+}$can be interpreted as "regard $k_{1}$ modulo $n$ ". To prove these facts, one can use the theorem of Baily and Borel (1966) to show that $\Gamma \backslash X^{+}$is algebraic, and a theorem of Borel (1972) to show that $\Gamma \backslash B$ is algebraic — see $\S 6$ where we discuss a similar question in greater detail.

REMARK 4.9. With the notations of the theorem, let $G$ be the algebraic group over $\mathbb{Q}$ such that

$$
G(\mathbb{Q})=\left\{g \in \mathrm{GL}_{E}(H) \mid \exists v(g) \in \mathbb{Q}^{\times} \text {such that } \psi(g x, g x)=v(g) \psi(x, y), \forall x, y \in H\right\} .
$$

The homomorphism $h: \mathbb{C}^{\times} \rightarrow \mathrm{GL}(H \otimes \mathbb{R})$ defined by the Hodge structure on $H_{1}(A, \mathbb{Q})$ factors through $G_{\mathbb{R}}$, and $X$ can be identified with the $G(\mathbb{R})$-conjugacy class of the homomorphisms $\mathbb{C}^{\times} \rightarrow G_{\mathbb{R}}$ containing $h$. Let $K$ be the compact open subgroup of $G\left(\mathbb{A}_{f}\right)$ of $g$ such 
that $(g-1) V(\hat{\mathbb{Z}}) \subset n V(\hat{\mathbb{Z}})$. Then $\Gamma \backslash X^{+}$is a connected component of the Shimura variety $\mathrm{Sh}_{K}(G, X)$. The general theory shows that $\operatorname{Sh}_{K}(G, X)$ is a fine moduli scheme (see Deligne $1971 \mathrm{c}, \S 4$, or Milne and Shih 1982, §2) and so, from this point of view, the only part of the above proof that is not immediate is that the connected component of $\operatorname{Sh}_{K}(G, X)$ containing $A$ also contains a variety $A_{0} \otimes E$.

REMARK 4.10. It is easy to construct algebraic cycles on $A_{0} \otimes E$ : every $\mathbb{Q}$-linear map $\lambda: E \rightarrow \mathbb{Q}$ defines a map $A_{0} \otimes E \rightarrow A_{0} \otimes \mathbb{Q}=A_{0}$, and we can take $\operatorname{cl}(\lambda)$ to be the image of the class of a point in $H^{d}\left(A_{0}\right) \rightarrow H^{d}\left(A_{0} \otimes E\right)$. More generally, we have

$$
\operatorname{Sym}^{*}\left(\operatorname{Hom}_{\mathbb{Q}-\text { linear }}(E, \mathbb{Q})\right) \rightarrow\left\{\text { algebraic cycles on } A_{0} \otimes E\right\} .
$$

If $E=\mathbb{Q}^{r}$, this gives the obvious cycles.

REMARK 4.11. The argument in the proof of Theorem 4.8 is similar to, and was suggested by, an argument of B. Gross (1978). 


\section{Completion of the proof for abelian varieties of CM- type}

\section{Abelian varieties of CM-type}

The Mumford-Tate, or Hodge, group of an abelian variety $A$ over $\mathbb{C}$ is defined to be the Mumford-Tate group of the rational Hodge structure $H_{1}(A, \mathbb{Q})$ : it is therefore the subgroup of $\mathrm{GL}\left(H_{1}(A, \mathbb{Q})\right) \times \mathbb{G}_{m}$ fixing all Hodge cycles on $A$ and its powers (see $\left.\S 3\right)$. In the language of Tannakian categories, the category of rational Hodge structures is Tannakian with an obvious fibre functor, and the Mumford-Tate group of $A$ is the group associated with the Tannakian subcategory generated by $H_{1}(A, \mathbb{Q})$ and $\mathbb{Q}(1)$.

An abelian variety $A$ is said to be of $\boldsymbol{C M}$-type if its Mumford-Tate group is commutative. Since every abelian variety $A$ is a product $A=\prod A_{\alpha}$ of simple abelian varieties (up to isogeny) and $A$ is of CM-type if and only if each $A_{\alpha}$ is of CM-type (the Mumford-Tate group of $A$ is contained in the product of the Mumford-Tate groups of the $A_{\alpha}$ and projects onto each), in understanding this concept we can assume $A$ is simple.

Proposition 5.1. A simple abelian variety $A$ over $\mathbb{C}$ is of CM-type if and only if $E=$ End $A$ is a commutative field over which $H_{1}(A, \mathbb{Q})$ has dimension 1 . Then $E$ is a $C M$-field, and the Rosati involution on $E=\operatorname{End}(A)$ defined by any polarization of $A$ is complex conjugation.

Proof. Let $A$ be an abelian variety such that $\operatorname{End}(A)$ contains a field $E$ for which $H_{1}(A, \mathbb{Q})$ has dimension 1 as an $E$-vector space. As $\mu\left(\mathbb{G}_{m}\right)$ commutes with $E \otimes \mathbb{R}$ in $\operatorname{End}\left(H_{1}(A, \mathbb{R})\right)$, we have that $\mu\left(\mathbb{G}_{m}\right) \subset(E \otimes \mathbb{R})^{\times}$and so the Mumford-Tate group of $A$ is contained in $E^{\times}$.

Conversely, let $A$ be simple and of CM-type, and let $\mu: \mathbb{G}_{m} \rightarrow \operatorname{GL}\left(H_{1}(A, \mathbb{C})\right)$ be defined by the Hodge structure on $H_{1}(A, \mathbb{C})$ (see $\S 3$ ). As $A$ is simple, $E=\operatorname{End}(A)$ is a field (possibly noncommutative) of degree $\leq \operatorname{dim} H_{1}(A, \mathbb{Q})$ over $\mathbb{Q}$. As for any abelian variety, $\operatorname{End}(A)$ is the subalgebra of $\operatorname{End}\left(H_{1}(A, \mathbb{Q})\right)$ of elements preserving the Hodge structure or, equivalently, that commute with $\mu\left(\mathbb{G}_{m}\right)$ in $\operatorname{GL}\left(H_{1}(A, \mathbb{C})\right)$. If $G$ is the Mumford-Tate group of $A$, then $G_{\mathbb{C}}$ is generated by the groups $\left\{\sigma \mu\left(\mathbb{G}_{m}\right) \mid \sigma \in \operatorname{Aut}(\mathbb{C})\right\}$ (see 3.4). Therefore $E$ is the commutant of $G$ in $\operatorname{End}\left(H_{1}(A, \mathbb{Q})\right)$. By assumption, $G$ is a torus, and so $H_{1}(A, \mathbb{C})=\bigoplus_{\chi \in X(G)} H_{\chi}$. The commutant of $G$ therefore contains étale commutative algebras of $\operatorname{rank} \operatorname{dim} H_{1}(A, \mathbb{Q})$ over $\mathbb{Q}$. It follows that $E$ is a commutative field of degree $\operatorname{dim} H_{1}(A, \mathbb{Q})$ over $\mathbb{Q}$ (and that it is generated as a $\mathbb{Q}$-algebra by $G(\mathbb{Q})$; in particular, $h(i) \in E \otimes \mathbb{R})$.

Let $\psi$ be a Riemann form corresponding to some polarization on $A$. The Rosati involution $e \mapsto e^{*}$ on $\operatorname{End}(A)=E$ is determined by the condition

$$
\psi(x, e y)=\psi\left(e^{*} x, y\right), \quad x, y \in H_{1}(A, \mathbb{Q}) .
$$

It follows from

$$
\psi(x, y)=\psi(h(i) x, h(i) y)
$$

that

$$
h(i)^{*}=h(i)^{-1} \quad(=-h(i)) .
$$

The Rosati involution therefore is nontrivial on $E$, and $E$ has degree 2 over its fixed field $F$. There exists an $\alpha \in F^{\times}$such that

$$
E=F[\sqrt{\alpha}], \quad \sqrt{\alpha}^{*}=-\sqrt{\alpha},
$$


and $\alpha$ is uniquely determined up to multiplication by a square in $F$. If $E$ is identified with $H_{1}(A, \mathbb{R})$ through the choice of an appropriate basis vector, then

$$
\psi(x, y)=\operatorname{Tr}_{E / \mathbb{Q}} \alpha x y^{*}, \quad x, y \in E,
$$

(cf. 4.6). The positivity condition on $\psi$ implies that

$$
\operatorname{Tr}_{E \otimes \mathbb{R} / \mathbb{R}}\left(f x^{2}\right)>0, \quad x \neq 0, \quad x \in F \otimes \mathbb{R}, \quad f=\alpha / h(i),
$$

which implies that $F$ is totally real. Moreover, for every embedding $\sigma: F \hookrightarrow \mathbb{R}$, we must have $\sigma(\alpha)<0$, for otherwise $E \otimes_{F, \sigma} \mathbb{R}=\mathbb{R} \times \mathbb{R}$ with $\left(r_{1}, r_{2}\right)^{*}=\left(r_{2}, r_{1}\right)$, and the positivity condition is impossible. Thus, $\sigma(\alpha)<0$, and $*$ is complex conjugation relative to any embedding of $E$ into $\mathbb{C}$. This completes the proof.

\section{Proof of the main theorem for abelian varieties of CM-type}

Let $\left(A_{\alpha}\right)$ be a finite family of abelian varieties over $\mathbb{C}$ of CM-type. We shall show that every element of a space

$$
T_{\mathbb{A}}=\left(\otimes_{\alpha} H_{\mathbb{A}}^{1}\left(X_{\alpha}\right)^{\otimes m_{\alpha}}\right) \otimes\left(\bigotimes_{\alpha} H_{\mathbb{A}}^{1}\left(X_{\alpha}\right)^{\vee \otimes n_{\alpha}}\right)(m)
$$

that is a Hodge cycle (relative to id: $\mathbb{C} \rightarrow \mathbb{C}$ ) is an absolute Hodge cycle. According to (3.8) (Principle A), to do this it suffices to show that the following two subgroups of $\mathrm{GL}\left(\prod H_{1}\left(A_{\alpha}, \mathbb{Q}\right)\right) \times \mathbb{G}_{m}$ are equal:

$$
\begin{aligned}
G^{H} & =\text { group fixing all Hodge cycles; } \\
G^{A H} & =\text { group fixing all absolute Hodge cycles. }
\end{aligned}
$$

Obviously $G^{H} \subset G^{A H}$.

After breaking up each $A_{\alpha}$ into its simple factors, we can assume $A_{\alpha}$ is itself simple. Let $E_{\alpha}$ be the CM-field $\operatorname{End}\left(A_{\alpha}\right)$ and let $E$ be the smallest Galois extension of $\mathbb{Q}$ containing all $E_{\alpha}$; it is again a CM-field. Let $B_{\alpha}=A_{\alpha} \otimes_{E_{\alpha}} E$. It suffices to prove the theorem for the family $\left(B_{\alpha}\right)$ (because the Tannakian category generated by the $H_{1}\left(B_{\alpha}\right)$ and $\mathbb{Q}(1)$ contains every $H_{1}\left(A_{\alpha}\right)$; cf. Deligne and Milne 1982).

In fact, we consider an even larger family. Fix $E$, a CM-field Galois over $\mathbb{Q}$, and consider the family $\left(A_{\alpha}\right)$ of all abelian varieties with complex multiplication by $E$ (so $H_{1}\left(A_{\alpha}\right)$ has dimension 1 over $E$ ) up to $E$-isogeny. This family is indexed by $\mathcal{S}$, the set of CM-types for $E$. Thus, if $S=\operatorname{Hom}(E, \mathbb{C})$, then each element of $\mathcal{S}$ is a set $\Phi \subset S$ such that $S=\Phi \sqcup \iota \Phi$ (disjoint union). We often identify $\Phi$ with the characteristic function of $\Phi$, i.e., we write

$$
\Phi(s)=\left\{\begin{array}{l}
1 \text { if } s \in \Phi \\
0 \text { if } s \notin \Phi
\end{array}\right.
$$

With each $\Phi$ we associate the isogeny class of abelian varieties containing the abelian variety $\mathbb{C}^{\Phi} / \Phi\left(\mathcal{O}_{E}\right)$ where $\mathcal{O}_{E}$ is the ring of integers in $E$ and

$$
\Phi\left(\mathcal{O}_{E}\right)=\left\{(\sigma e)_{\sigma \in \Phi} \in \mathbb{C}^{\Phi} \mid e \in \mathcal{O}_{E}\right\} .
$$

With this new family, we have to show that $G^{H}=G^{A H}$. We begin by determining $G^{H}$ (cf. 3.7). The Hodge structure on each $H_{1}\left(A_{\Phi}, \mathbb{Q}\right)$ is compatible with the action of $E$. This 
implies that, as a subgroup of $\prod_{\Phi \in \mathcal{S}} \operatorname{GL}\left(H_{1}\left(A_{\Phi}\right)\right) \times \mathbb{G}_{m}, G^{H}$ commutes with $\prod_{\Phi \in \mathcal{S}} E^{\times}$ and is therefore contained in $\prod E^{\times} \times \mathbb{G}_{m}$. In particular, $G^{H}$ is a torus and can be described by its group of cocharacters $Y\left(G^{H}\right) \stackrel{\text { def }}{=} \operatorname{Hom}_{\mathbb{Q}^{a l}}\left(\mathbb{G}_{m}, G^{H}\right)$ or its group of characters $X\left(G^{H}\right)$. Note that

$$
Y\left(G^{H}\right) \subset Y\left(\prod_{\Phi \in \mathcal{S}} E^{\times} \times \mathbb{G}_{m}\right)=\mathbb{Z}^{S \times \mathcal{S}} \times \mathbb{Z} .
$$

There is a canonical basis for $X\left(E^{\times}\right)$, namely the set $S$, and therefore a canonical basis for $X\left(\prod_{\Phi \in \mathcal{S}} E^{\times} \times \mathbb{G}_{m}\right)$ which we denote $\left(\left(x_{s, \Phi}\right), x_{0}\right)$. We denote the dual basis for $Y\left(\prod E^{\times} \times\right.$ $\left.\mathbb{G}_{m}\right)$ by $\left(y_{s, \Phi}, y_{0}\right)$. The element $\mu \in Y\left(G^{H}\right)$ equals $\sum_{s, \Phi} \Phi(s) y_{s, \Phi}+y_{0}$ (see 3.7). As $G_{\mathbb{C}}^{H}$ is generated by $\left\{\sigma \mu\left(\mathbb{G}_{m}\right) \mid \sigma \in \operatorname{Aut}(\mathbb{C})\right\}, Y\left(G^{H}\right)$ is the $\operatorname{Gal}\left(\mathbb{Q}^{\text {al }} / \mathbb{Q}\right)$-submodule of $Y\left(\prod E^{\times} \times \mathbb{G}_{m}\right)$ generated by $\mu$. (Here, $\operatorname{Gal}\left(\mathbb{Q}^{\text {al }} / \mathbb{Q}\right)$ acts on $S$ by $\sigma s=s \circ \sigma^{-1}$; it acts on $Y\left(\prod_{\Phi \in \mathcal{S}} E^{\times} \times \mathbb{G}_{m}\right)=\mathbb{Z}^{S \times \mathcal{S}} \times \mathbb{Z}$ through its action on $S, \sigma y_{s, \Phi}=y_{\sigma s, \Phi}$; these actions factor through $\operatorname{Gal}(E / \mathbb{Q}))$.

To begin the computation of $G^{A H}$, we make a list of the tensors we know to be absolute Hodge cycles on the $A_{\alpha}$.

(a) The endomorphisms $E \subset \operatorname{End}\left(A_{\Phi}\right)$ for each $\Phi$. (More precisely, we mean the classes $c l_{\mathbb{A}}\left(\Gamma_{e}\right) \in H_{\mathbb{A}}\left(A_{\Phi}\right) \otimes H_{\mathbb{A}}\left(A_{\Phi}\right), \Gamma_{e}=$ graph of $\left.e, e \in E.\right)$

(b) Let $\left(A_{\Phi}, v: E \hookrightarrow \operatorname{End}\left(A_{\Phi}\right)\right)$ correspond to $\Phi \in \mathcal{S}$, and let $\sigma \in \operatorname{Gal}(E / \mathbb{Q})$. Define $\sigma \Phi=\{\sigma s \mid s \in \Phi\}$. There is an isomorphism $A_{\Phi} \rightarrow A_{\sigma \Phi}$ induced by

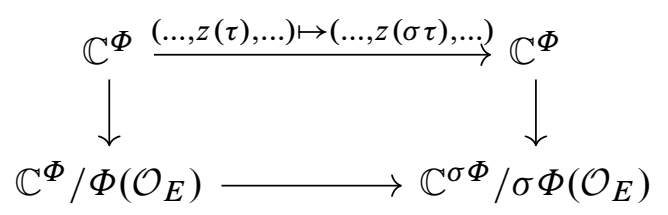

whose graph is an absolute Hodge cycle. (Alternatively, we could have used the fact that $\left(A_{\Phi}, \sigma v: E \rightarrow \operatorname{End}\left(A_{\Phi}\right)\right)$, where $\sigma v=v \circ \sigma^{-1}$, is of type $\sigma \Phi$ to show that $A_{\Phi}$ and $A_{\sigma \Phi}$ are isomorphic.)

(c) Let $\left(\Phi_{i}\right)_{1 \leq i \leq d}$ be a family of elements of $\mathcal{S}$ and let $A=\bigoplus_{i=1}^{d} A_{i}$ where $A_{i}=A_{\Phi_{i}}$. Then $E$ acts on $A$ and $H_{1}(A, \mathbb{Q})=\bigoplus_{i=1}^{d} H_{1}\left(A_{i}, \mathbb{Q}\right)$ has dimension $d$ over $E$. Under the assumption that $\sum_{i} \Phi_{i}=$ constant (so that $\sum_{i} \Phi_{i}(s)=d / 2$, all $s \in S$ ), we shall apply (4.8) to construct absolute Hodge cycles on $A$.

For each $i$, there is an $E$-linear isomorphism

$$
H_{1}\left(A_{i}, \mathbb{Q}\right) \otimes_{\mathbb{Q}} \mathbb{C} \rightarrow \bigoplus_{s \in S} H_{1}\left(A_{i}\right)_{s}
$$

such that $s \in E$ acts on $H_{1}\left(A_{i}\right)_{s}$ as $s(e)$. From the definitions one sees that

$$
H_{1}\left(A_{i}\right)_{s}= \begin{cases}H_{1}\left(A_{i}\right)_{s}^{-1,0}, & s \in \Phi_{i}, \\ H_{1}\left(A_{i}\right)_{s}^{0,-1}, & s \notin \Phi_{i} .\end{cases}
$$

Thus, with the notations of (4.4),

$$
\begin{aligned}
& a_{s}=\sum_{i} \Phi_{i}(s) \\
& b_{s}=\sum_{i}\left(1-\Phi_{i}(s)\right)=\sum_{i} \Phi_{i}(\iota s)=a_{\iota s} .
\end{aligned}
$$

The assumption that $\sum \Phi_{i}=$ constant therefore implies that

$$
a_{s}=b_{s}=d / 2, \quad \text { all } s .
$$


For each $i$, choose a polarization $\theta_{i}$ for $A_{i}$ whose Rosati involution stabilizes $E$, and let $\psi_{i}$ be the corresponding Riemann form. For any totally positive elements $f_{i}$ in $F$ (the maximal totally real subfield of $E$ ) $\theta=\bigoplus_{i} f_{i} \theta_{i}$ is a polarization for $A$. Choose $v_{i} \neq 0$, $v_{i} \in H_{1}\left(A_{i}, \mathbb{Q}\right)$; then $\left\{v_{i}\right\}$ is a basis for $H_{1}\left(A_{i}, \mathbb{Q}\right)$ over $E$. There exist $\zeta_{i} \in E^{\times}$such that $\bar{\zeta}_{i}=-\zeta_{i}$ and $\psi_{i}\left(x v_{i}, y v_{i}\right)=\operatorname{Tr}_{E / \mathbb{Q}}\left(\zeta_{i} x \bar{y}\right)$ for all $x, y \in E$. Thus $\phi_{i}$, where $\phi_{i}\left(x v_{i}, y v_{i}\right)=$ $\frac{\zeta_{i}}{\zeta_{1}} x \bar{y}$, is an $E$-Hermitian form on $H_{1}\left(A_{i}, \mathbb{Q}\right)$ such that $\psi_{i}(v, w)=\operatorname{Tr}_{E / \mathbb{Q}}\left(\zeta_{1} \phi_{i}(v, w)\right)$. The $E$-Hermitian form on $H_{1}(A, \mathbb{Q})$

$$
\phi\left(\sum x_{i} v_{i}, \sum y_{i} v_{i}\right)=\sum_{i} f_{i} \phi_{i}\left(x_{i} v_{i}, y_{i} v_{i}\right)
$$

has the property that $\psi(v, w) \stackrel{\text { def }}{=} \operatorname{Tr}_{E / \mathbb{Q}}\left(\zeta_{1} \phi(v, w)\right)$ and is the Riemann form of $\theta$. The discriminant of $\phi$ is $\prod_{i} f_{i}\left(\frac{\zeta_{i}}{\zeta_{1}}\right)$. On the other hand, if $s \in S$ restricts to $\tau$ on $F$, then

$$
\operatorname{sign}(\tau \operatorname{disc}(\phi))=(-1)^{b_{s}}=(-1)^{d / 2} .
$$

Thus,

$$
\operatorname{disc}(\phi)=(-1)^{d / 2} f
$$

for some totally positive element $f$ of $F$. After replacing one $f_{i}$ with $f_{i} / f$, we have that $\operatorname{disc}(\phi)=(-1)^{d / 2}$, and that $\phi$ is split. Hence (4.8) applies.

In summary: let $A=\oplus_{i=1}^{d} A_{\Phi_{i}}$ be such that $\sum_{i} \Phi_{i}=$ constant; then

$$
\left(\bigwedge_{E}^{d} H^{1}(A, \mathbb{Q})\left(\frac{d}{2}\right)\right) \subset H^{d}(A, \mathbb{Q})\left(\frac{d}{2}\right)
$$

consists of absolute Hodge cycles.

Since $G^{A H}$ fixes the absolute Hodge cycles of type (a), $G^{A H} \subset \prod_{\Phi} E^{\times} \times \mathbb{G}_{m}$. It is therefore a torus, and we have an inclusion

$$
Y\left(G^{A H}\right) \subset Y\left(\prod E^{\times} \times \mathbb{G}_{m}\right)=\mathbb{Z}^{S \times \mathcal{S}} \times \mathbb{Z}
$$

and a surjection,

$$
X\left(\prod E^{\times} \times \mathbb{G}_{m}\right)=\mathbb{Z}^{S \times \mathcal{S}} \times \mathbb{Z} \rightarrow X\left(G^{A H}\right) .
$$

Let $W$ be a space of absolute Hodge cycles. The action of the torus $\prod E^{\times} \times \mathbb{G}_{m}$ on $W \otimes \mathbb{C}$ decomposes it into a sum $\oplus W_{\chi}$ indexed by the $\chi \in X\left(\prod E^{\times} \times \mathbb{G}_{m}\right)$ of subspaces $W_{\chi}$ on which the torus acts through $\chi$. Since $G^{A H}$ fixes the elements of $W$, the $\chi$ for which $W_{\chi} \neq 0$ map to zero in $X\left(G^{A H}\right)$.

On applying this remark with $W$ equal to the space of absolute Hodge cycles described in (b), we find that $x_{s, \Phi}-x_{\sigma s, \sigma \Phi}$ maps to zero in $X\left(G^{A H}\right)$, all $\sigma \in \operatorname{Gal}(E / \mathbb{Q}), s \in S$, and $\Phi \in \mathcal{S}$. As $\operatorname{Gal}(E / \mathbb{Q})$ acts simply transitively on $S$, this implies that, for a fixed $s_{0} \in S$, $X\left(G^{A H}\right)$ is generated by the image of $\left\{x_{S_{0}, \Phi}, x_{0} \mid \Phi \in \mathcal{S}\right\}$.

Let $d(\Phi) \geq 0$ be integers such that $\sum d(\Phi) \Phi=d / 2$ (constant function on $S$ ) where $d=\sum d(\Phi)$. Then (c) shows that the subspace

$$
W \stackrel{\text { def }}{=} \bigotimes_{E} H_{1}\left(A_{\Phi}, \mathbb{Q}\right)^{\otimes_{E} d(\Phi)}(-d / 2)=\bigwedge_{E}^{d} H_{1}\left(\bigoplus A_{\Phi}^{d(\Phi)}, \mathbb{Q}\right)(-d / 2)
$$

of $H_{d}\left(\bigoplus A_{\Phi}^{d(\Phi)}, \mathbb{Q}\right)(-d / 2)$ consists of absolute Hodge cycles. The remark then shows that $\sum d(\Phi) x_{s, \Phi}-d / 2$ maps to zero in $X\left(G^{A H}\right)$ for all $s$.

Let

$$
X=X\left(\prod E^{\times} \times \mathbb{G}_{m}\right) / \sum \mathbb{Z}\left(x_{\sigma s, \sigma \Phi}-x_{s, \Phi}\right)
$$


and regard

$$
\left\{x_{s_{0}, \Phi}, x_{0} \mid \Phi \in \mathcal{S}\right\}
$$

as a basis for $X$. We know that

$$
X\left(\prod E^{\times} \times \mathbb{G}_{m}\right) \rightarrow X\left(G^{A H}\right)
$$

factors through $X$, and that therefore $Y \supset Y\left(G^{A H}\right)\left(\supset Y\left(G^{H}\right)\right)$ where $Y$ is the submodule of $Y\left(\prod E^{\times} \times \mathbb{G}_{m}\right)$ dual to $X$.

Lemma 5.2. The submodule $Y\left(G^{H}\right)^{\perp}$ of $X$ orthogonal to $Y\left(G^{H}\right)$ is equal to

$$
\left\{\sum d(\Phi) x_{s_{0}, \Phi}-\frac{d}{2} x_{0} \mid \sum d(\Phi) \Phi=\frac{d}{2}, \sum d(\Phi)=d\right\} ;
$$

it is generated by the elements

$$
\sum d(\Phi) x_{s_{0}, \Phi}-\frac{d}{2} x_{0}, \quad \sum d(\Phi) \Phi=\frac{d}{2}, \quad d(\Phi) \geq 0 \text { all } \Phi .
$$

Proof. As $Y\left(G^{H}\right)$ is the $\operatorname{Gal}(E / \mathbb{Q})$-submodule of $Y$ generated by $\mu$, we see that

$$
x=\sum d(\Phi) x_{s_{0}, \Phi}-\frac{d}{2} x_{0} \in Y\left(G^{H}\right)^{\perp}
$$

if and only if $\langle\sigma \mu, x\rangle=0$ all $\sigma \in \operatorname{Gal}(E / \mathbb{Q})$. But $\mu=\sum \Phi(s) y_{s, \Phi}+y_{0}$ and $\sigma \mu=$ $\sum \Phi(s) y_{\sigma s, \Phi}+x_{0}$, and so $\langle\sigma \mu, x\rangle=\sum d(\Phi) \Phi\left(\sigma^{-1} s_{0}\right)-\frac{d}{2}$. The first assertion is now obvious.

As $\Phi+\iota \Phi=1, x_{s_{0}, \Phi}+x_{s_{0}, \iota \Phi}-x_{0} \in Y\left(G^{H}\right)^{\perp}$ and has positive coefficients $d(\Phi)$. By adding enough elements of this form to an arbitrary element $x \in Y\left(G^{H}\right)^{\perp}$ we obtain an element with coefficients $d(\Phi) \geq 0$, which completes the proof of the lemma.

The lemma shows that $Y\left(G^{H}\right)^{\perp} \subset \operatorname{Ker}\left(X \rightarrow X\left(G^{A H}\right)\right)=Y\left(G^{A H}\right)^{\perp}$. Hence $Y\left(G^{H}\right) \subset$ $Y\left(G^{A H}\right)$ and it follows that $G^{H}=G^{A H}$; the proof is complete. 


\section{Completion of the proof; consequences}

\section{Completion of the proof of Theorem 2.11}

Let $A$ be an abelian variety over $\mathbb{C}$ and let $t_{\alpha}, \alpha \in I$, be Hodge cycles on $A$ (relative to id: $\mathbb{C} \rightarrow \mathbb{C}$ ). To prove the Main Theorem 2.11, we have to show that the $t_{\alpha}$ are absolute Hodge cycles. Since we know the result for abelian varieties of CM-type, Theorem 2.15 shows that it remains only to prove the following proposition.

PROPOSITION 6.1. There exists a connected smooth algebraic variety $S$ over $\mathbb{C}$ and an abelian scheme $\pi: Y \rightarrow S$ such that

(a) for some $s_{0} \in S, Y_{s_{0}}=A$;

(b) for some $s_{1} \in S, Y_{s_{1}}$ is of CM-type;

(c) the $t_{\alpha}$ extend to elements that are rational and of bidegree $(0,0)$ everywhere in the family.

The last condition means the following. Suppose that $t_{\alpha}$ belongs to a tensor space $T_{\alpha}=H_{\mathrm{B}}^{1}(A)^{\otimes m(\alpha)} \otimes \ldots ;$ then there is a section $t$ of $\left(R^{1} \pi_{*} \mathbb{Q}\right)^{\otimes m(\alpha)} \otimes \ldots$ over the universal covering $\tilde{S}$ of $S$ (equivalently, over a finite covering of $S$ ) such that for $\tilde{s}_{0}$ mapping to $s_{0}$, $t_{\tilde{s}_{0}}=t_{\alpha}$, and for all $\tilde{s} \in \tilde{S}, t_{\tilde{s}} \in H_{\mathrm{B}}^{1}\left(Y_{\tilde{S}}\right)^{\otimes m(\alpha)} \otimes \ldots$ is a Hodge cycle.

We sketch a proof of Proposition 6.1. The parameter variety $S$ will be a Shimura variety and (b) will hold for a dense set of points $s_{1}$.

We may suppose that one of the $t_{\alpha}$ is a polarization $\theta$ for $A$. Let $H=H_{1}(A, \mathbb{Q})$ and let $G$ be the subgroup of $\operatorname{GL}(H) \times \mathbb{G}_{m}$ fixing the $t_{\alpha}$. The Hodge structure on $H$ defines a homomorphism $h_{0}: \mathbb{C}^{\times} \rightarrow G(\mathbb{R})$. Let $G^{0}=\operatorname{Ker}\left(G \rightarrow \mathbb{G}_{m}\right)$; then $\operatorname{ad}\left(h_{0}(i)\right)$ is a Cartan involution on $G_{\mathbb{C}}^{0}$ because the real form of $G_{\mathbb{C}}^{0}$ corresponding to it fixes the positive definite form $\psi(x, h(i) y)$ on $H \otimes \mathbb{R}$ where $\psi$ is a Riemann form for $\theta$. In particular, $G$ is reductive (see 3.6).

Let

$$
X=\left\{h: \mathbb{C}^{\times} \rightarrow G(\mathbb{R}) \mid h \text { is conjugate to } h_{0} \text { under } G(\mathbb{R})\right\} .
$$

Each $h \in X$ defines a Hodge structure on $H$ of type $\{(-1,0),(0,-1)\}$ relative to which each $t_{\alpha}$ is of bidegree $(0,0)$. Let $F^{0}(h)=H^{0,-1} \subset H \otimes \mathbb{C}$. Since $G(\mathbb{R}) / K_{\infty} \stackrel{\approx}{\rightarrow} X$, where $K_{\infty}$ is the centralizer of $h_{0}$, there is an obvious real differentiable structure on $X$, and the tangent space to $X$ at $h_{0}, \operatorname{Tgt}_{h_{0}}(X)=\operatorname{Lie}\left(G_{\mathbb{R}}\right) / \operatorname{Lie}\left(K_{\infty}\right)$. In fact, $X$ is a Hermitian symmetric domain. The Grassmannian,

$$
\operatorname{Grass}_{d}(H \otimes \mathbb{C}) \stackrel{\text { def }}{=}\{W \subset H \otimes \mathbb{C} \mid W \text { of dimension } d \quad(=\operatorname{dim} A)\}
$$

is a complex analytic manifold (even an algebraic variety). The map

$$
\phi: X \rightarrow \operatorname{Grass}_{d}(H \otimes \mathbb{C}), \quad h \mapsto F^{0}(h),
$$

is a real differentiable map, and is injective (because the Hodge filtration determines the Hodge decomposition). The map on tangent spaces factors into

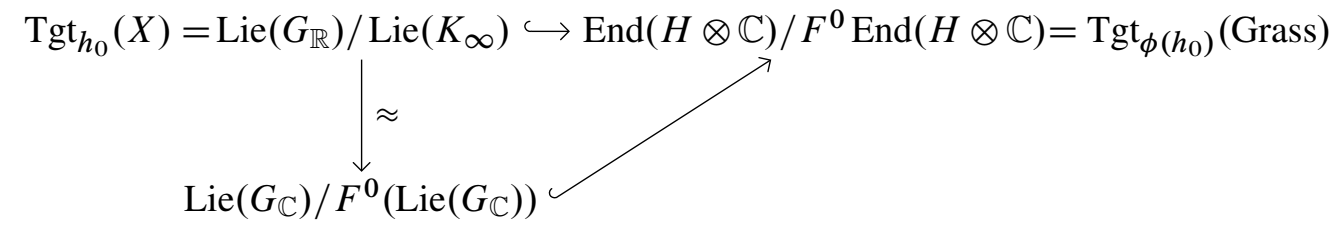


— the maps are induced by $G(\mathbb{R}) \hookrightarrow G(\mathbb{C}) \hookrightarrow \operatorname{GL}(H \otimes \mathbb{C})$ and the filtrations on $\operatorname{Lie}\left(G_{\mathbb{C}}\right)$ and $\operatorname{End}(H \otimes \mathbb{C})$ are those corresponding to the Hodge structure defined by $h_{0}$. Thus, $d \phi$ identifies $\operatorname{Tgt}_{h_{0}}(X)$ with a complex subspace of $\operatorname{Tgt}_{\phi\left(h_{0}\right)}(\mathrm{Grass})$, and so $X$ is an almostcomplex (in fact, complex) manifold (see Deligne 1979b, 1.1, for more details). (There is an alternative, more group-theoretic, description of the complex structure; see Knapp 1972, 2.4, 2.5.)

To each point $h$ of $X$, we can attach a complex torus $F^{0}(h) \backslash H \otimes \mathbb{C} / H(\mathbb{Z})$, where $H(\mathbb{Z})$ is some fixed lattice in $H$. For example, to $h_{0}$ is attached

$$
F^{0}\left(h_{0}\right) \backslash H \otimes \mathbb{C} / H(\mathbb{Z})=\operatorname{Tgt}_{0}(A) / H(\mathbb{Z}),
$$

which is an abelian variety representing $A$. From the definition of the complex structure on $X$, it is clear that these tori form an analytic family $B$ over $X$.

Let

$$
\Gamma=\{g \in G(\mathbb{Q}) \mid(g-1) H(\mathbb{Z}) \subset n H(\mathbb{Z})\}
$$

some fixed integer $n$. For a suitably large $n \geq 3, \Gamma$ will act freely on $X$, and so $\Gamma \backslash X$ will again be a complex manifold. The theorem of Baily and Borel (1966) shows that $S=\Gamma \backslash X$ is an algebraic variety.

The group $\Gamma$ acts compatibly on $B$, and on forming the quotients, we obtain a complex analytic map $\pi: Y \rightarrow S$ with $Y=\Gamma \backslash B$. For $s \in S, Y_{S}$ is a polarized complex torus (hence an abelian variety) with level $n$ structure (induced by $H_{1}\left(B_{h}, \mathbb{Z}\right) \stackrel{\approx}{\rightarrow} H(\mathbb{Z})$ where $h$ maps to $s$ ). The solution $M_{n}$ of the moduli problem for polarized abelian varieties with level $n$-structure in the category of algebraic varieties is also a solution in the category of complex analytic manifolds. There is therefore an analytic map $\psi: S \rightarrow M_{n}$ such that $Y$ is the pull-back of the universal family on $M_{n}$. A theorem of Borel $(1972,3.10)$ shows that $\psi$ is automatically algebraic, from which it follows that $Y / S$ is an algebraic family.

For some connected component $S^{\circ}$ of $S, \pi^{-1}\left(S^{\circ}\right) \rightarrow S^{\circ}$ will satisfy (a) and (c) of the proposition. To prove (b) we shall show that, for some $h \in X$ close to $h_{0}, B_{h}$ is of CM-type (cf. Deligne 1971c, 5.2).

Recall (§5) that an abelian variety is of CM-type if and only if its Mumford-Tate group is a torus. From this it follows that $B_{h}, h \in X$, is of CM-type if and only if $h$ factors through the real points of a subtorus of $G$ defined over $\mathbb{Q}$.

Let $T$ be a maximal torus, defined over $\mathbb{R}$, of the algebraic group $K_{\infty}$. (See Borel and Springer 1966 for a proof that $T$ exists, or apply the argument that follows.) Since $h_{0}\left(\mathbb{C}^{\times}\right)$ is contained in the centre of $K_{\infty}, h_{0}\left(\mathbb{C}^{\times}\right) \subset T(\mathbb{R})$. If $T^{\prime}$ is a torus in $G_{\mathbb{R}}$ containing $T$, then $T^{\prime}$ will centralize $h_{0}$ and so $T^{\prime} \subset K_{\infty} ; T$ is therefore maximal in $G_{\mathbb{R}}$. For a general (regular) element $\lambda$ of $\operatorname{Lie}(T), T$ is the centralizer of $\lambda$. Choose a $\lambda_{0} \in \operatorname{Lie}(G)$ that is close to $\lambda$ in $\operatorname{Lie}\left(G_{\mathbb{R}}\right)$, and let $T_{0}$ be the centralizer of $\lambda_{0}$ in $G$. Then $T_{0}$ is a maximal torus of $G$ that is defined over $\mathbb{Q}$, and, because $T_{0 \mathbb{R}}$ is close to $T_{\mathbb{R}}, T_{0 \mathbb{R}}=g T g^{-1}$ for some $g \in G(\mathbb{R})$. Now $h=\operatorname{ad}(g) \circ h_{0}$ factors through $T_{0 \mathbb{R}}$, and so $B_{h}$ is of CM-type.

This completes the proof of the main theorem.

\section{Consequences of Theorem 2.11}

We end this section by giving two immediate consequences.

Let $X$ be a complete smooth variety over a field $k$ and let $\gamma \in H^{2 p}\left(X_{\mathrm{et}}, \mathbb{Q}_{\ell}\right)(p), \ell \neq$ $\operatorname{char}(k)$. Tate's conjecture states that $\gamma$ is in the $\mathbb{Q}_{\ell}$-span of the algebraic classes if there 
exists a subfield $k_{0}$ of $k$ finitely generated over the prime field, a model $X_{0}$ of $X$ over $k_{0}$, and a $\gamma \in H^{2 p}\left(X_{0} \otimes \bar{k}_{0}, \mathbb{Q}_{\ell}\right)(p)$ mapping to $\gamma$ that is fixed by $\operatorname{Gal}\left(\bar{k}_{0} / k_{0}\right)$.

Corollary 6.2. Let $A$ be an abelian variety over $\mathbb{C}$. If Tate's conjecture is true for $A$, then so also is the Hodge conjecture.

Proof. We first show that, for any complete smooth variety $X$ over $\mathbb{C}$, Tate's conjecture implies that all absolute Hodge cycles on $X$ are algebraic. Let $X_{0}$ be a model of $X$ over a subfield $k_{0}$ of $\mathbb{C}$ finitely generated over $\mathbb{Q}$. According to Proposition 2.9, $C_{\mathrm{AH}}^{p}(X)=$ $C_{\mathrm{AH}}^{p}\left(X_{0} \otimes \bar{k}_{0}\right)$ and, after we have replaced $k_{0}$ by a finite extension, $\operatorname{Gal}\left(\bar{k}_{0} / k_{0}\right)$ will act trivially on $C_{\mathrm{AH}}^{p}\left(X_{0} \otimes \bar{k}_{0}\right)$. Let $C_{\mathrm{alg}}^{p}(X)$ denote the $\mathbb{Q}$-subspace of $C_{\mathrm{AH}}^{p}(X)$ spanned by the algebraic cycles on $X$. Tate's conjecture implies that the $\mathbb{Q}_{\ell}$-span of $C_{\mathrm{AH}}^{p}(X)$ is contained in the $\mathbb{Q}_{\ell}$-span of $C_{\mathrm{alg}}^{p}(X)$. Hence $C_{\mathrm{alg}}^{p}(X) \otimes \mathbb{Q}_{\ell}=C_{\mathrm{AH}}^{p}(X) \otimes \mathbb{Q}_{\ell}$, and so $C_{\mathrm{alg}}^{p}(X)=C_{\mathrm{AH}}^{p}(X)$.

Now let $A$ be an abelian variety over $\mathbb{C}$, and let $t \in H^{2 p}(A, \mathbb{Q}) \cap H^{p, p}$. The image $t^{\prime}$ of $t$ in $H_{\mathbb{A}}^{2 p}(A)(p)$ is a Hodge cycle relative to id: $\mathbb{C} \rightarrow \mathbb{C}$, and so Theorem 2.11 shows that $t^{\prime} \in C_{\mathrm{AH}}^{p}(A)$. It is therefore in the $\mathbb{Q}$-span of the algebraic cycles.

REMARK 6.3. The last result was first proved independently by Pjateckii Šapiro 1971 and Deligne (unpublished) by an argument similar to that which concluded the proof of the main theorem. (Corollary 6.2 is easy to prove for abelian varieties of CM-type; in fact, Pohlmann 1968 shows that the two conjectures are equivalent in that case.) We mention also that Borovoi 1977 shows that, for an abelian variety $X$ over a field $k$, the $\mathbb{Q}_{\ell}$-subspace of $H^{2 p}\left(X_{\mathrm{et}}, \mathbb{Q}_{\ell}\right)(p)$ generated by cycles that are Hodge relative to an embedding $\sigma: k \hookrightarrow \mathbb{C}$ is independent of the embedding.

Corollary 6.4. Let $A$ be an abelian variety over $\mathbb{C}$ and let $G^{A}$ be the Mumford-Tate group of $A$. Then $\operatorname{dim}\left(G^{A}\right) \geq \operatorname{tr} \cdot \operatorname{deg}_{k} k\left(p_{i j}\right)$ where $p_{i j}$ are the periods of $A$.

Proof. Same as that of Proposition 1.6. 


\section{Algebraicity of values of the $\Gamma$-function}

The next result generalizes Proposition 1.5.

Proposition 7.1. Let $\bar{k}$ be an algebraically closed subfield of $\mathbb{C}$, and let $V$ be a complete smooth variety of dimension $n$ over $\bar{k}$. If $\sigma \in H_{2 r}^{B}(V)$ maps to an absolute Hodge cycle $\gamma$ under

$$
H_{2 r}^{B}(V) \stackrel{1 \mapsto(2 \pi i)^{-r}}{\longrightarrow} H_{2 r}^{B}(V)(-r) \stackrel{\simeq}{\rightarrow} H_{\mathrm{B}}^{2 n-2 r}(V)(n-r) \hookrightarrow H_{\mathbb{A}}^{2 n-2 r}\left(V_{\mathbb{C}}\right)(n-r)
$$

then, for any $C^{\infty}$ differential $r$-form $\omega$ on $V_{\mathbb{C}}$ whose class $[\omega]$ in $H_{\mathrm{dR}}^{2 r}(V / \mathbb{C})$ lies in $H_{\mathrm{dR}}^{2 r}(V / \bar{k})$,

$$
\int_{\sigma} \omega \in(2 \pi i)^{r} \bar{k}
$$

Proof. Proposition 2.9 shows that $\gamma$ arises from an absolute Hodge cycle $\gamma_{0}$ on $V / \bar{k}$. Let $\left(\gamma_{0}\right)_{\mathrm{dR}}$ be the component of $\gamma_{0}$ in $H_{\mathrm{dR}}^{2 n-2 r}(V / \bar{k})$. Then, as in the proof of (1.5),

$$
\int_{\sigma} \omega=(2 \pi i)^{r} \operatorname{Tr}_{\mathrm{dR}}\left(\left(\gamma_{0}\right)_{\mathrm{dR}} \cup[\omega]\right) \in(2 \pi i)^{r} H_{\mathrm{dR}}^{2 n}(V / \bar{k})=(2 \pi i)^{r} \bar{k} .
$$

In the most important case of the proposition, $k$ will be the algebraic closure $\overline{\mathbb{Q}}$ of $\mathbb{Q}$ in $\mathbb{C}$, and it will then be important to know not only that the period

$$
P(\sigma, \omega) \stackrel{\text { def }}{=}(2 \pi i)^{-r} \int_{\sigma} \omega
$$

is algebraic, but also in which field it lies in. We begin by describing a general procedure for finding this field and then illustrate it by an example in which $V$ is a Fermat hypersurface and the period is a product of values of the $\Gamma$-function.

Let $V$ now be a complete smooth variety over a number field $k \subset \mathbb{C}$, and let $S$ be a finite abelian group acting on $V$ over $k$. Let $\bar{V}=V \otimes_{k} \overline{\mathbb{Q}}$. When $\alpha: S \rightarrow \mathbb{C}^{\times}$is a character of $S$ taking values in $k^{\times}$and $H$ is a $k$-vector space on which $S$ acts, we let

$$
H_{\alpha}=\{v \in H \mid s v=\alpha(s) v, \text { all } s \in S\} .
$$

Assume that all Hodge cycles on $V_{\mathbb{C}}$ are absolutely Hodge and that $H^{2 r}(V(\mathbb{C}), \mathbb{C})_{\alpha}$ has dimension 1 and is of bidegree $(r, r)$. Then $\left(C_{\mathrm{AH}}^{r}(\bar{V}) \otimes k\right)_{\alpha}$ has dimension one over $k$. The actions of $S$ and $\mathrm{Gal}(\overline{\mathbb{Q}} / k)$ on $H_{\mathrm{dR}}^{2 r}(\bar{V} / \overline{\mathbb{Q}}) \simeq H_{\mathrm{dR}}^{2 r}(V / k) \otimes_{k} \overline{\mathbb{Q}}$ commute because the latter acts through its action on $\overline{\mathbb{Q}}$; they therefore also commute on $C_{\mathrm{AH}}^{r}(\bar{V}) \otimes k$, which embeds into $H_{\mathrm{dR}}^{2 r}(\bar{V} / \overline{\mathbb{Q}})$. It follows that $\mathrm{Gal}(\overline{\mathbb{Q}} / k)$ stabilizes $\left(C_{\mathrm{AH}}^{r}(\bar{V}) \otimes k\right)_{\alpha}$ and, as this has dimension 1 , there is a character $\chi: \operatorname{Gal}(\overline{\mathbb{Q}} / k) \rightarrow k^{\times}$such that

$$
\tau \gamma=\chi(\tau)^{-1} \gamma, \quad \tau \in \operatorname{Gal}(\overline{\mathbb{Q}} / k), \quad \gamma \in\left(C_{\mathrm{AH}}^{r}(\bar{V}) \otimes k\right)_{\alpha} .
$$

Proposition 7.2. With the above assumptions, let $\sigma \in H_{2 r}^{B}(V)$ and let $\omega$ be a $C^{\infty}$ differential $2 r$-form on $V(\mathbb{C})$ whose class $[\omega]$ in $H_{\mathrm{dR}}^{2 r}(V / \mathbb{C})$ lies in $H_{\mathrm{dR}}^{2 r}(V / k)_{\alpha}$; then $P(\sigma, \omega)$ lies in an abelian algebraic extension of $k$, and

$$
\tau(P(\sigma, \omega))=\chi(\tau) P(\sigma, \omega) \text { for all } \tau \in \operatorname{Gal}(\overline{\mathbb{Q}} / k) .
$$


Proof. Consider $[\omega] \in H_{\mathrm{dR}}^{2 r}(V / \mathbb{C})_{\alpha}=\left(C_{\mathrm{AH}}^{r}(\bar{V}) \otimes \mathbb{C}\right)_{\alpha}$; then $[\omega]=z \gamma$ for some $z \in \mathbb{C}$, $\gamma \in\left(C_{\mathrm{AH}}^{r}(\bar{V}) \otimes k\right)_{\alpha}$. Moreover,

$$
P(\sigma, \omega) \stackrel{\text { def }}{=}\left(\frac{1}{2 \pi i}\right)^{r} \int_{\sigma} \omega=z \gamma\left(\sigma \otimes(2 \pi i)^{-r}\right) \in z k
$$

where we are regarding $\gamma$ as an element of $H_{\mathrm{B}}^{2 r}(V)(r) \otimes k=H_{2 r}^{B}(V)(-r)^{\vee} \otimes k$. Thus

$$
P(\sigma, \omega)^{-1}[\omega] \in C_{\mathrm{AH}}^{r}(\bar{V}) \otimes k .
$$

As

$$
[\omega] \in H_{\mathrm{dR}}^{2 r}(\bar{V} / \overline{\mathbb{Q}})=C_{\mathrm{AH}}^{r}(\bar{V}) \otimes \overline{\mathbb{Q}},
$$

this shows that $P(\sigma, \omega) \in \overline{\mathbb{Q}}$. Moreover,

$$
\tau\left(P(\sigma, \omega)^{-1}[\omega]\right)=\chi(\tau)^{-1}\left(P(\sigma, \omega)^{-1}[\omega]\right) .
$$

On using that $\tau[\omega]=[\omega]$, we deduce that

$$
\tau(P(\sigma, \omega))=\chi(\tau) \cdot P(\sigma, \omega) .
$$

REMARK 7.3. (a) Because $C_{\mathrm{AH}}^{r}(\bar{V})$ injects into $H^{2 r}\left(\bar{V}_{\mathrm{et}}, \mathbb{Q}_{\ell}\right)(r), \chi$ can be calculated from the action of $\operatorname{Gal}(\overline{\mathbb{Q}} / k)$ on $H^{2 r}\left(\bar{V}_{\text {et }}, \mathbb{Q}_{\ell}\right)_{\alpha}(r)$.

(b) The argument in the proof of the proposition shows that $\sigma \otimes(2 \pi i)^{-r} \in H_{2 r}^{B}(V)(-r)$ and $P(\sigma, \omega)^{-1}[\omega] \in H_{\mathrm{dR}}^{2 r}(\bar{V} / \overline{\mathbb{Q}})$ are different manifestations of the same absolute Hodge cycle.

\section{The Fermat hypersurface}

We shall apply (7.2) to the Fermat hypersurface

$$
V: X_{0}^{d}+X_{1}^{d}+\cdots+X_{n+1}^{d}=0
$$

of degree $d$ and dimension $n$, which we shall regard as a variety over $k \stackrel{\text { def }}{=} \mathbb{Q}\left(e^{2 \pi i / d}\right)$. As above, we let $\bar{V}=V \otimes_{k} \overline{\mathbb{Q}}$, and we shall often drop the the subscript on $V_{\mathbb{C}}$.

It is known that the motive of $V$ is contained in the category of motives generated by abelian varieties (see Deligne and Milne 1982, 6.26), and therefore Theorem 2.11 shows that every Hodge cycle on $V$ is absolutely Hodge (ibid. 6.27).

Let $\mu_{d}$ be the group of $d$ th roots of 1 in $\mathbb{C}$, and let

$$
S=\bigoplus_{i=0}^{n+1} \mu_{d} / \text { (diagonal) }
$$

Then $S$ acts on $V / k$ according to the formula:

$$
\left(\ldots: \zeta_{i}: \ldots\right)\left(\ldots: x_{i}: \ldots\right)=\left(\ldots: \zeta_{i} x_{i}: \ldots\right), \quad \text { all }\left(\ldots: x_{i}: \ldots\right) \in V(\mathbb{C})
$$

The character group of $S$ will be identified with

$$
X(S)=\left\{\mathbf{a} \in(\mathbb{Z} / d \mathbb{Z})^{n+2} \mid \mathbf{a}=\left(a_{0}, \ldots, a_{n+1}\right), \quad \sum_{i} a_{i}=0\right\} ;
$$


here $\mathbf{a} \in X(S)$ corresponds to the character

$$
\underline{\zeta}=\left(\zeta_{0}: \ldots\right) \mapsto \underline{\zeta}^{\mathbf{a}} \stackrel{\text { def }}{=} \prod_{i=0}^{n+1} \zeta_{i}^{a_{i}} .
$$

For $a \in \mathbb{Z} / d \mathbb{Z}$, we let $\langle a\rangle$ denote the representative of $a$ in $\mathbb{Z}$ with $1 \leq\langle a\rangle \leq d$, and for $\mathbf{a} \in X(S)$ we let $\langle\mathbf{a}\rangle=d^{-1} \sum\left\langle a_{i}\right\rangle \in \mathbb{N}$.

If $H(V)$ is a cohomology group on which there is a natural action of $k$, we have a decomposition

$$
H(V)=\bigoplus H(V)_{\mathbf{a}}, \quad H(V)_{\mathbf{a}}=\left\{v \mid \underline{\zeta} v=\underline{\zeta}^{\mathbf{a}} v, \quad \underline{\zeta} \in S\right\} .
$$

Let $(\mathbb{Z} / d \mathbb{Z})^{\times}$act on $X(S)$ in the obvious way,

$$
u \cdot\left(a_{0}, \ldots\right)=\left(u a_{0}, \ldots\right),
$$

and let $[\mathbf{a}]$ denote the orbit of a. The irreducible representations of $S$ over $\mathbb{Q}$ (and hence the idempotents of $\mathbb{Q}[S]$ ) are classified by the these orbits, and so $\mathbb{Q}[S]=\prod \mathbb{Q}[\mathbf{a}]$ where $\mathbb{Q}[\mathbf{a}]$ is a field whose degree over $\mathbb{Q}$ is equal to the order of $[\mathbf{a}]$. The map $\underline{\xi} \mapsto \zeta^{\mathbf{a}}: S \rightarrow \mathbb{C}$ induces an embedding $\mathbb{Q}[\mathbf{a}] \hookrightarrow k$. Every cohomology group decomposes as $H(V)=\bigoplus H(V)_{[\mathbf{a}]}$ where

$$
H(V)_{[\mathbf{a}]} \otimes \mathbb{C}=\bigoplus_{\mathbf{a}^{\prime} \in[\mathbf{a}]}(H(V) \otimes \mathbb{C})_{\mathbf{a}^{\prime}}
$$

\section{Calculation of the cohomology}

Proposition 7.4. The dimension of $H^{n}(V, \mathbb{C})_{\mathbf{a}}$ is 1 if no $a_{i}=0$ or if all $a_{i}=0$ and $n$ is even; otherwise $H^{n}(V, \mathbb{C})_{\mathbf{a}}=0$.

Proof. The map

$$
\left(x_{0}: x_{1}: \ldots\right) \mapsto\left(x_{0}^{d}: x_{1}^{d}: \ldots\right): \mathbb{P}^{n+1} \rightarrow \mathbb{P}^{n+1}
$$

defines a finite surjective map $\pi: V \rightarrow \mathbb{P}^{n}$ where $P^{n} \quad\left(\approx \mathbb{P}^{n}\right)$ is the hyperplane $\sum X_{i}=0$. There is an action of $S$ on $\pi_{*} \mathbb{C}$, which induces a decomposition $\pi_{*} \mathbb{C} \simeq \bigoplus\left(\pi_{*} \mathbb{C}\right)_{\mathbf{a}}$. The isomorphism $H^{r}(V, \mathbb{C}) \stackrel{\simeq}{\rightarrow} H^{r}\left(P^{n}, \pi_{*} \mathbb{C}\right)$ is compatible with the actions of $S$, and so gives rise to isomorphisms

$$
H^{r}(V, \mathbb{C})_{\mathbf{a}} \stackrel{\simeq}{\rightarrow} H^{r}\left(P^{n},\left(\pi_{*} \mathbb{C}\right)_{\mathbf{a}}\right) .
$$

Clearly $\left(\pi_{*} \mathbb{C}\right)_{0}=\mathbb{C}$, and so

$$
H^{r}\left(P^{n},\left(\pi_{*} \mathbb{C}\right)_{0}\right) \simeq H^{r}\left(\mathbb{P}^{n}, \mathbb{C}\right) \text { for all } r .
$$

For $\mathbf{a} \neq \mathbf{0}$, the sheaf $\left(\pi_{*} \mathbb{C}\right)_{\mathbf{a}}$ is locally constant of dimension 1, except over the hyperplanes $H_{i}: X_{i}=0$ corresponding to the $i$ for which $a_{i} \neq 0$, where it is ramified. It follows that

$$
H^{r}\left(P^{n},\left(\pi_{*} \mathbb{C}\right)_{\mathbf{a}}\right)=0, \quad r \neq n, \quad \mathbf{a} \neq \mathbf{0},
$$

and so $(-1)^{n} \operatorname{dim} H^{n}\left(P^{n},\left(\pi_{*} \mathbb{C}\right)_{\mathbf{a}}\right)$ is equal to the Euler-Poincaré characteristic of $\left(\pi_{*} \mathbb{C}\right)_{\mathbf{a}}$ $(\mathbf{a} \neq 0)$. We have

$$
\operatorname{EP}\left(P^{n},\left(\pi_{*} \mathbb{C}\right)_{\mathbf{a}}\right)=\operatorname{EP}\left(P^{n} \backslash \bigcup_{a_{i} \neq 0} H_{i}, \mathbb{C}\right) .
$$


Suppose first that no $a_{i}$ is zero. Then

$$
\left(x_{0}: \ldots: x_{n}:-\sum x_{i}\right) \leftrightarrow\left(x_{0}: \ldots: x_{n}\right): P^{n} \stackrel{\widetilde{\longrightarrow}}{\leftrightarrow} \mathbb{P}^{n}
$$

induces

$$
P^{n} \backslash \bigcup_{i=0}^{n+1} H_{i} \underset{\leftrightarrow}{\stackrel{0}{\leftrightarrow}} \mathbb{P}^{n} \backslash \bigcup_{i=0}^{n} H_{i} \cup P^{n-1},
$$

where $H_{i}$ denotes the coordinate hyperplane in $\mathbb{P}^{n+1}$ or $\mathbb{P}^{n}$. As

$$
\left(\mathbb{P}^{n} \backslash \bigcup H_{i} \cup P^{n-1}\right) \sqcup\left(P^{n-1} \backslash \bigcup H_{i}\right)=\mathbb{P}^{n} \backslash \bigcup H_{i},
$$

and $\mathbb{P}^{n} \backslash \bigcup H_{i}$, being topologically isomorphic to $\left(\mathbb{C}^{\times}\right)^{n}$, has Euler-Poincaré characteristic zero, we see that

$$
\operatorname{EP}\left(P^{n} \backslash \bigcup^{n+1} H_{i}\right)=-\operatorname{EP}\left(P^{n-1} \backslash \bigcup^{n} H_{i}\right)=\ldots=(-1)^{n} \operatorname{EP}\left(P^{0}\right)=(-1)^{n} .
$$

If some, but not all, $a_{i}$ are zero, then $P^{n} \backslash \bigcup H_{i} \approx\left(\mathbb{C}^{\times}\right)^{r} \times \mathbb{C}^{n-r}$ with $r \geq 1$, and so $\operatorname{EP}\left(P^{n} \backslash \bigcup H_{i}\right)=0^{r} \times 1^{n-r}=0$.

REMARK 7.5. Note that the above argument shows that the primitive cohomology of $V$,

$$
H^{n}(V, \mathbb{C})_{\text {prim }}=\bigoplus_{\mathbf{a} \neq \mathbf{0}} H^{n}(V, \mathbb{C})_{\mathbf{a}}
$$

The action of $S$ on $H^{n}(V, \mathbb{C})$ respects the Hodge decomposition, and so $H^{n}(V, \mathbb{C})_{\mathbf{a}}$ is purely of bidegree $(p, q)$ for some $p, q$ with $p+q=n$.

Proposition 7.6. If no $a_{i}=0$, then $H^{n}(V, \mathbb{C})_{\mathbf{a}}$ is of bidegree $(p, q)$ with $p=\langle\mathbf{a}\rangle-1$.

ProOF. We apply the method of Griffiths 1969 , $\S 8$. When $V$ is a smooth hypersurface in $\mathbb{P}^{n+1}$, Griffiths shows that the maps in

$$
H^{n+1}\left(\mathbb{P}^{n+1}, \mathbb{C}\right) \stackrel{0}{\longrightarrow} H^{n+1}\left(\mathbb{P}^{n+1} \backslash V, \mathbb{C}\right) \longrightarrow H_{V}^{n+2}\left(\mathbb{P}^{n+1}, \mathbb{C}\right) \longrightarrow H^{n+2}\left(\mathbb{P}^{n+1}, \mathbb{C}\right)
$$

induce an isomorphism

$$
H^{n+1}\left(\mathbb{P}^{n+1} \backslash V, \mathbb{C}\right) \stackrel{\simeq}{\rightarrow} H^{n}(V)(-1)_{\text {prim }}
$$

and that the Hodge filtration on $H^{n}(V)(-1)$ has the following explicit interpretation: identify $H^{n+1}\left(\mathbb{P}^{n+1} \backslash V, \mathbb{C}\right)$ with $\Gamma\left(\mathbb{P}^{n+1} \backslash V, \Omega^{n+1}\right) / d \Gamma\left(\mathbb{P}^{n+1} \backslash V, \Omega^{n}\right)$ and let

$$
\Omega_{p}^{n+1}(V)=\left\{\omega \in \Gamma\left(\mathbb{P}^{n+1} \backslash V, \Omega^{n+1}\right) \mid \omega \text { has a pole of order } \leq p \text { on } V\right\} ;
$$

then the map

$$
R: \Omega_{p}^{n+1}(V) \rightarrow H^{n}(V, \mathbb{C})
$$

determined by

$$
\langle\sigma, R(\omega)\rangle=\frac{1}{2 \pi i} \int_{\sigma} \omega, \quad \text { all } \sigma \in H_{n}(V, \mathbb{C}),
$$


induces an isomorphism

$$
\Omega_{p}^{n+1}(V) / d \Omega_{p-1}^{n} \stackrel{\approx}{\rightarrow} F^{n-p} H^{n}(V)(-1)_{\text {prim }}=F^{n-p+1} H^{n}(V)_{\text {prim }}
$$

(For example, when $p=1, R$ is the residue map

$$
\left.\Omega_{1}^{n+1}(V) \rightarrow F^{n} H^{n}(V)=H^{0}\left(V, \Omega^{n}\right)\right) .
$$

Let $f$ be the irreducible polynomial definining $V$. As $\Omega_{\mathbb{P}^{n+1}}^{n+1}(n+2) \approx \mathcal{O}_{\mathbb{P}^{n+1}}$ has basis

$$
\omega_{0}=\sum(-1)^{i} X_{i} d X_{0} \wedge \ldots \wedge \widehat{d X_{i}} \wedge \ldots \wedge d X_{n},
$$

any differential form $\omega$ equals $P \omega_{0} / f^{p}$ with $P$ a homogeneous polynomial of degree $p \operatorname{deg}(f)-(n+2)$ lies in $\Omega_{p}^{n+1}(V)$. In particular, when $V$ is our Fermat surface,

$$
\begin{aligned}
\omega & =\frac{X_{0}^{\left\langle a_{0}\right\rangle-1} \cdots X_{n+1}^{\left\langle a_{n+1}\right\rangle-1}}{\left(X_{0}^{d}+\cdots+X_{n+1}^{d}\right)^{\langle\mathbf{a}\rangle}} \omega_{0} \\
& =\frac{X_{0}^{\left\langle a_{0}\right\rangle} \cdots X_{n+1}^{\left\langle a_{n+1}\right\rangle}}{\left(X_{0}^{d}+\cdots+X_{n+1}^{d}\right)^{\langle\mathbf{a}\rangle}} \sum(-1)^{i} \frac{d X_{0}}{X_{0}} \wedge \ldots \wedge \frac{\widehat{d X_{i}}}{X_{i}} \wedge \ldots
\end{aligned}
$$

lies in $\Omega_{\langle\mathbf{a}\rangle}^{n+1}(V)$. For $\underline{\zeta} \in S, \underline{\zeta} X_{i}=\zeta_{i}^{-1} X_{i}$, and so $\underline{\zeta} \omega=\zeta^{-\mathbf{a}} \omega$. This shows that

$$
H^{n}(V, \mathbb{C})_{-\mathbf{a}} \subset F^{n-\langle\mathbf{a}\rangle+1} H^{n}(V, \mathbb{C}) .
$$

Since $\langle-\mathbf{a}\rangle-1=n+1-\langle\mathbf{a}\rangle$, we can rewrite this inclusion as

$$
H^{n}(V, \mathbb{C})_{\mathbf{a}} \subset F^{\langle\mathbf{a}\rangle-1} H^{n}(V, \mathbb{C}) .
$$

Thus $H^{n}(V, \mathbb{C})_{\mathbf{a}}$ is of bidegree $(p, q)$ with $p \geq\langle\mathbf{a}\rangle-1$. The complex conjugate of $H^{n}(V, \mathbb{C})_{\mathbf{a}}$ is $H^{n}(V, \mathbb{C})_{-\mathbf{a}}$, and is of bidegree $(q, p)$. Hence

$$
n-p=q \geq\langle-\mathbf{a}\rangle-1=n+1-\langle\mathbf{a}\rangle
$$

and so $p \leq\langle\mathbf{a}\rangle-1$.

Recall that $H_{\mathrm{B}}^{n}(V)_{[\mathrm{a}]}=\bigoplus_{\mathbf{a}^{\prime} \in[\mathbf{a}]} H_{\mathrm{B}}^{n}(V)_{\mathbf{a}^{\prime}}$; thus (7.4) shows that $H_{\mathrm{B}}^{n}(V)_{[\mathbf{a}]}$ has dimension 1 over $\mathbb{Q}[\mathbf{a}]$ when no $a_{i}$ is zero and otherwise

$$
H_{\mathrm{B}}^{n}(V)_{[\mathbf{a}]} \cap H_{\mathrm{B}}^{n}(V)_{\text {prim }}=0 .
$$

COROLlaRY 7.7. Let a be such that no $a_{i}=0$. Then $H_{\mathrm{B}}^{n}(V)_{[\mathrm{a}]}$ is purely of type $\left(\frac{n}{2}, \frac{n}{2}\right)$ if and only if $\langle u \mathbf{a}\rangle$ is independent of $u$.

Proof. As $\langle\mathbf{a}\rangle+\langle-\mathbf{a}\rangle=n+2,\langle u \mathbf{a}\rangle$ is constant if and only if $\langle u \mathbf{a}\rangle=\frac{n}{2}+1$ for all $u \in$ $(\mathbb{Z} / d \mathbb{Z})^{\times}$, i.e., if and only if $\left\langle\mathbf{a}^{\prime}\right\rangle=\frac{n}{2}+1$ for all $\mathbf{a}^{\prime} \in[\mathbf{a}]$. Thus the corollary follows from the proposition.

COROLlaRY 7.8. If no $a_{i}=0$ and $\langle u \mathbf{a}\rangle$ is constant, then $C_{\mathrm{AH}}^{n}(\bar{V})_{[\mathrm{a}]}$ has dimension one over $\mathbb{Q}[\mathbf{a}]$.

ProOF. This follows immediately from (7.7) because, as we have remarked, all Hodge cycles on $V$ are absolutely Hodge. 


\section{The action of $\mathrm{Gal}(\overline{\mathbb{Q}} / k)$ on the étale cohomology}

Let $\mathfrak{p}$ be a prime ideal of $k$ not dividing $d$, and let $\mathbb{F}_{q}$ be the residue field of $\mathfrak{p}$. Then $d \mid q-1$ and reduction modulo $\mathfrak{p}$ defines an isomorphism $\mu_{d} \rightarrow \mathbb{F}_{d}^{\times}$whose inverse we denote $t$. Fix an $\mathbf{a}=\left(a_{0}, \ldots, a_{n+1}\right) \in X(S)$ with all $a_{i}$ nonzero, and define a character $\varepsilon_{i}: \mathbb{F}_{q}^{\times} \rightarrow \mu_{d}$ by

$$
\varepsilon_{i}(x)=t\left(x^{(1-q) / d}\right)^{a_{i}}, \quad x \neq 0 .
$$

As $\prod \varepsilon_{i}=1$, the product $\prod \varepsilon_{i}\left(x_{i}\right)$ is well-defined for $\mathbf{x}=\left(x_{0}: \ldots: x_{n+1}\right) \in \mathbb{P}^{n+1}\left(\mathbb{F}_{q}\right)$, and we define a Jacobi sum

$$
J\left(\varepsilon_{0}, \ldots, \varepsilon_{n+1}\right)=(-1)^{n} \sum_{\mathbf{x} \in P^{n}\left(\mathbb{F}_{q}\right)} \prod_{i=0}^{n+1} \varepsilon_{i}\left(x_{i}\right)
$$

where $P^{n}$ is the hyperplane $\sum X_{i}=0$ in $\mathbb{P}^{n+1}$. (As usual, we set $\varepsilon_{i}(0)=0$.) Let $\psi$ be a nontrivial additive character $\psi: \mathbb{F}_{q} \rightarrow \mathbb{C}^{\times}$and define Gauss sums

$$
\begin{aligned}
g\left(\mathfrak{p}, a_{i}, \psi\right) & =-\sum_{x \in \mathbb{F}_{q}} \varepsilon_{i}(x) \psi(x) \\
g(\mathfrak{p}, \mathbf{a}) & =q^{-\langle\mathbf{a}\rangle} \prod_{i=0}^{n+1} g\left(\mathfrak{p}, a_{i}, \psi\right) .
\end{aligned}
$$

LEMma 7.9. The Jacobi sum $J\left(\varepsilon_{0}, \ldots, \varepsilon_{n+1}\right)=q^{\langle\mathbf{a}\rangle-1} g(\mathfrak{p}, \mathbf{a})$.

PROOF. We have

$$
\begin{aligned}
q^{\langle\mathbf{a}\rangle} g(\mathfrak{p}, \mathbf{a}) & =\prod_{i=0}^{n+1}\left(-\sum_{x \in \mathbb{F}_{q}} \varepsilon_{i}(x) \psi(x)\right) \\
& =(-1)^{n} \sum_{\mathbf{x} \in \mathbb{F}_{q}^{n+2}}\left(\prod_{i=0}^{n+1} \varepsilon_{i}\left(x_{i}\right)\right) \psi\left(\sum x_{i}\right), \quad \mathbf{x}=\left(x_{0}, \ldots\right) \\
& =(-1)^{n} \sum_{\mathbf{x} \in \mathbb{P}^{n+1}\left(\mathbb{F}_{q}\right)} \sum_{\lambda \in \mathbb{F}_{q}^{\times}}\left(\prod_{i=0}^{n+1} \varepsilon_{i}\left(\lambda x_{i}\right) \psi\left(\lambda \sum x_{i}\right)\right) .
\end{aligned}
$$

We can omit the $\lambda$ from $\prod \varepsilon_{i}\left(\lambda x_{i}\right)$, and so obtain

$$
q^{\langle\mathbf{a}\rangle} g(\mathfrak{p}, \mathbf{a})=(-1)^{n} \sum_{\mathbf{x} \in \mathbb{P}^{n+1}\left(\mathbb{F}_{q}\right)}\left(\prod_{i=0}^{n+1} \varepsilon_{i}\left(x_{i}\right) \cdot \sum_{\lambda \in \mathbb{F}_{q}^{\times}}\left(\psi\left(\lambda \sum x_{i}\right)\right)\right) .
$$

Since

$$
\left.\sum_{\mathbf{x}} \prod_{i=0}^{n+1} \varepsilon_{i}\left(x_{i}\right)=\prod_{i=0}^{n+1}\left(\sum_{x \in \mathbb{F}_{q}} \varepsilon_{i}(x)\right)\right)=0,
$$

we can replace the sum over $\lambda \in \mathbb{F}_{q}^{\times}$by a sum over $\lambda \in \mathbb{F}_{q}$. From

$$
\sum_{\lambda \in \mathbb{F}_{q}} \psi\left(\lambda \sum x_{i}\right)= \begin{cases}q & \text { if } \sum x_{i}=0 \\ 0 & \text { if } \sum x_{i} \neq 0\end{cases}
$$

we deduce finally that

$$
\begin{aligned}
q^{\langle\mathbf{a}\rangle} g(\mathfrak{p}, \mathbf{a}) & \left.=(-1)^{n} q \sum_{\mathbf{x} \in P^{n}\left(\mathbb{F}_{q}\right)} \prod_{i=0}^{n+1} \varepsilon_{i}\left(x_{i}\right)\right) \\
& =q J\left(\varepsilon_{0}, \ldots, \varepsilon_{n}\right) .
\end{aligned}
$$


Note that this shows that $g(\mathfrak{p}, \mathbf{a})$ is independent of $\psi$ and lies in $k$.

Let $\ell$ be a prime number such that $\ell \nmid d, \mathfrak{p} \nmid \ell$, and $d \mid \ell-1$. Then $\mathbb{Q}_{\ell}$ contains a primitive $d$ th root of 1 and so, after choosing an embedding $k \hookrightarrow \mathbb{Q}_{\ell}$, we can assume $g(\mathfrak{p}, \mathbf{a}) \in \mathbb{Q}_{\ell}$.

Proposition 7.10. Let $F_{\mathfrak{p}} \in \operatorname{Gal}(\mathbb{Q} / k)^{\mathrm{ab}}$ be a geometric Frobenius element of $\mathfrak{p} \nmid d$; for all $v \in H^{n}\left(\bar{V}_{\mathrm{et}}, \mathbb{Q}_{\ell}\right)_{\mathbf{a}}$,

$$
F_{\mathfrak{p}} v=q^{\langle\mathbf{a}\rangle-1} g(\mathfrak{p}, \mathbf{a}) v .
$$

Proof. As $\mathfrak{p} \nmid d, V$ reduces to a smooth variety $V_{\mathfrak{p}}$ over $\mathbb{F}_{q}$ and the proper-smooth base change theorem shows that there is an isomorphism $H^{n}\left(\bar{V}, \mathbb{Q}_{\ell}\right) \rightarrow H^{n}\left(\bar{V}_{\mathfrak{p}}, \mathbb{Q}_{\ell}\right)$ compatible with the action of $S$ and carrying the action of $F_{\mathfrak{p}}$ on $H^{n}\left(\bar{V}, \mathbb{Q}_{\ell}\right)$ into the action of the Frobenius endomorphism Frob on $H^{n}\left(\bar{V}_{\mathfrak{p}}, \mathbb{Q}_{\ell}\right)$. The comparison theorem shows that $H^{n}\left(\bar{V}, \mathbb{Q}_{\ell}\right)_{\mathbf{a}}$ has dimension 1 , and so it remains to compute

$$
\operatorname{Tr}\left(F_{\mathfrak{p}} \mid H^{n}\left(\bar{V}, \mathbb{Q}_{\ell}\right)_{\mathbf{a}}\right)=\operatorname{Tr}\left(\operatorname{Frob} \mid H^{n}\left(\bar{V}_{\mathfrak{p}}, \mathbb{Q}_{\ell}\right)_{\mathbf{a}}\right) .
$$

Let $\pi: V_{\mathfrak{p}} \rightarrow P^{n}$ be as before. Then

$$
H^{n}\left(\bar{V}_{\mathfrak{p}}, \mathbb{Q}_{\ell}\right)_{\mathbf{a}}=H^{n}\left(P^{n},\left(\pi_{*} \mathbb{Q}_{\ell}\right)_{\mathbf{a}}\right),
$$

and the Lefschetz trace formula shows that

$$
(-1)^{n} \operatorname{Tr}\left(\operatorname{Frob} \mid H^{n}\left(P^{n},\left(\pi_{*} \mathbb{Q}_{\ell}\right)_{\mathbf{a}}\right)=\sum_{\mathbf{x} \in P^{n}(\mathbb{F})} \operatorname{Tr}\left(\operatorname{Frob} \mid\left(\left(\pi_{*} \mathbb{Q}_{\ell}\right)_{\mathbf{a}}\right)_{\mathbf{x}}\right)\right.
$$

where $\left(\left(\pi_{*} \mathbb{Q}_{\ell}\right)_{\mathbf{a}}\right)_{\mathbf{x}}$ is the stalk of $\left(\pi_{*} \mathbb{Q}_{\ell}\right)_{\mathbf{a}}$ at $\mathbf{x}$.

Fix an $\mathbf{x} \in P^{n}\left(\mathbb{F}_{q}\right)$ with no $x_{i}$ zero, and let $\mathbf{y} \in V_{\mathfrak{p}}\left(\overline{\mathbb{F}}_{q}\right)$ map to $\mathbf{x}$; thus $y_{i}^{d}=x_{i}$ all $i$. Then $\pi^{-1}(\mathbf{x})=\{\underline{\zeta} \mathbf{y} \mid \underline{\zeta} \in S\}$, and $\left(\pi_{*} \mathbb{Q}_{\ell}\right)_{\mathbf{x}}$ is the vector space $\mathbb{Q}_{\ell}^{\pi^{-1}(\mathbf{x})}$.

If $\phi$ denotes the arithmetic Frobenius automorphism (i.e., the generator $z \mapsto z^{q}$ of $\left.\operatorname{Gal}\left(\overline{\mathbb{F}}_{q} / \mathbb{F}_{q}\right)\right)$, then

$$
\phi\left(y_{i}\right)=y_{i}^{q}=x_{i}^{\frac{q-1}{d}} y_{i}=t\left(x_{i}^{\frac{q-1}{d}}\right) y_{i}, \quad 0 \leq i \leq n-1,
$$

and so

$$
\operatorname{Frob}(\mathbf{y})=\underline{\eta} \mathbf{y} \text { where } \underline{\eta}=\left(\ldots: t\left(x_{i}^{\frac{1-q}{d}}\right): \ldots\right) \in S .
$$

Thus Frob acts on $\left(\pi_{*} \mathbb{Q}_{\ell}\right)_{\mathbf{x}}$ as $\underline{\eta}$, and for $v \in\left(\left(\pi_{*} \mathbb{Q}_{\ell}\right)_{\mathbf{a}}\right)_{\mathbf{X}}$, we have

$$
\operatorname{Frob}(v)=\underline{\eta} v=\underline{\eta}^{\mathbf{a}_{v}}, \quad \underline{\eta}^{\mathbf{a}}=\prod_{i=0}^{n+1} \varepsilon_{i}\left(x_{i}\right) \in k \subset \mathbb{Q}_{\ell} .
$$

Consequently,

$$
\operatorname{Tr}\left(\operatorname{Frob} \mid\left(\left(\pi_{*} \mathbb{Q}_{\ell}\right)_{\mathbf{a}}\right)_{\mathbf{x}}\right)=\prod_{i=0}^{n+1} \varepsilon_{i}\left(x_{i}\right) .
$$

If some $x_{i}=0$, then both sides are zero $\left(\left(\pi_{*} \mathbb{Q}_{\ell}\right)_{\mathbf{a}}\right.$ is ramified over the coordinate hyperplanes), and so, on summing over $\mathbf{x}$ and applying (2) and (7.9), we obtain the proposition.

COROLlary 7.11. Let a be such that no $a_{i}$ is zero and $\langle u \mathbf{a}\rangle$ is constant. Then, for all $v \in H^{n}\left(\bar{V}_{\mathrm{et}}, \mathbb{Q}_{\ell}\right)_{\mathbf{a}}\left(\frac{n}{2}\right)$,

$$
F_{\mathfrak{p}} v=g(\mathfrak{p}, \mathbf{a}) v .
$$

Proof. The hypotheses on a imply that $\langle\mathbf{a}\rangle=\frac{n}{2}+1$. Therefore, when we write $v=v_{0} \otimes 1$ with $v_{0} \in H^{n}\left(\bar{V}_{\text {et }}, \mathbb{Q}_{\ell}\right)_{\mathbf{a}}$,

$$
F_{\mathfrak{p}} v=F_{\mathfrak{p}} v_{0} \otimes F_{\mathfrak{p}} 1=q^{\frac{n}{2}} g(\mathfrak{p}, \mathbf{a}) v_{0} \otimes q^{\frac{-n}{2}}=g(\mathfrak{p}, \mathbf{a}) v .
$$




\section{Calculation of the periods}

Recall that that the $\Gamma$-function is defined by

$$
\Gamma(s)=\int_{0}^{\infty} e^{-t} t^{s} \frac{d t}{t}, \quad s>0,
$$

and satisfies the following equations

$$
\begin{gathered}
\Gamma(s) \Gamma(1-s)=\pi(\sin \pi s)^{-1} \\
\Gamma(1+s)=s \Gamma(s) .
\end{gathered}
$$

The last equation shows that, for $s \in \mathbb{Q}^{\times}$, the class of $\Gamma(s)$ in $\mathbb{C} / \mathbb{Q}^{\times}$depends only on the class of $s$ in $\mathbb{Q} / \mathbb{Z}$. Thus, for $\mathbf{a} \in X(S)$, we can define

$$
\tilde{\Gamma}(\mathbf{a})=(2 \pi i)^{-\langle\mathbf{a}\rangle} \prod_{i=0}^{n+1} \Gamma\left(\frac{a_{i}}{d}\right) \in \mathbb{C} / \mathbb{Q}^{\times} .
$$

Let $V^{o}$ denote the open affine subvariety of $V$ with equation

$$
Y_{1}^{d}+\cdots+Y_{n-1}^{d}=-1 \quad\left(\text { so } Y_{i}=X_{i} / X_{0}\right) .
$$

Denote by $\Delta$ the $n$-simplex

$$
\left\{\left(t_{1}, \ldots, t_{n+1}\right) \in \mathbb{R}^{n+1} \mid t_{i} \geq 0, \quad \sum t_{i}=1\right\}
$$

and define $\sigma_{0}: \Delta \rightarrow V^{o}(\mathbb{C})$ to be

$$
\left(t_{1}, \ldots, t_{n+1}\right) \mapsto\left(\varepsilon t_{1}^{\frac{1}{d}}, \ldots, \varepsilon t_{n+1}^{\frac{1}{d}}\right), \quad \varepsilon=e^{2 \pi i / 2 d}=\sqrt[d]{-1}, \quad t_{i}^{\frac{1}{d}}>0 .
$$

LeMmA 7.12. Let $a_{0}, \ldots, a_{n+1}$ be positive integers such that $\sum a_{i} \equiv 0 \bmod d$. Then

$$
\int_{\sigma_{0}(\Delta)} Y_{1}^{a_{1}} \cdots Y_{n+1}^{a_{n+1}} \frac{d Y_{1}}{Y_{1}} \wedge \ldots \wedge \frac{d Y_{n}}{Y_{n}}=\frac{1}{2 \pi i}\left(1-\xi^{-a_{0}}\right) \prod_{i=0}^{n+1} \Gamma\left(\frac{a_{i}}{d}\right)
$$

where $\xi=e^{2 \pi i / d}$.

PROOF. Write $\omega_{0}$ for the integrand. Then

$$
\begin{aligned}
\int_{\sigma_{0}(\Delta)} \omega_{0} & =\int_{\Delta} \sigma_{0}^{*}\left(\omega_{0}\right) \\
& =\int_{\Delta}\left(\varepsilon t_{1}^{\frac{1}{d}}\right)^{a_{1}} \cdots\left(\varepsilon t_{n+1}^{\frac{1}{d}}\right)^{a_{n+1}} d^{-n} \frac{d t_{1}}{t_{1}} \wedge \ldots \wedge \frac{d t_{n}}{t_{n}} \\
& =c \int_{\Delta} t_{1}^{b_{1}} \cdots t_{n+1}^{b_{n+1}} \frac{d t_{1}}{t_{1}} \wedge \ldots \wedge \frac{d t_{n}}{n}
\end{aligned}
$$

where $b_{i}=a_{i} / d$ and $c=\varepsilon^{a_{1}+\cdots+a_{n+1}}\left(\frac{1}{d}\right)^{n}$. On multiplying by

$$
\Gamma\left(1-b_{0}\right)=\Gamma\left(1+b_{1}+\cdots+b_{n+1}\right)=\int_{0}^{\infty} e^{-t} t^{b_{1}+\cdots+b_{n+1}} d t
$$


we obtain

$$
\Gamma\left(1-b_{0}\right) \int_{\sigma_{0}(\Delta)} \omega_{0}=c \int_{0}^{\infty} \int_{\Delta} e^{-t} t^{b_{1}+\cdots+b_{n+1}} t_{1}^{b_{1}} \cdots t_{n+1}^{b_{n+1}} \frac{d t_{1}}{t_{1}} \wedge \ldots \wedge \frac{d t_{n}}{n} \wedge d t .
$$

If, on the inner integral, we make the change of variables $s_{i}=t t_{i}$, the integral becomes

$$
c \int_{0}^{\infty} \int_{\Delta(t)} e^{-t} s_{1}^{b_{1}} \ldots s_{n+1}^{b_{n+1}} \frac{d s_{1}}{s_{1}} \wedge \ldots \wedge \frac{d s_{n}}{s_{n}} \wedge d t
$$

where

$$
\Delta(t)=\left\{\left(s_{1}, \ldots, s_{n+1}\right) \mid s_{i} \geq 0, \quad \sum s_{i}=t\right\} .
$$

We now let $t=\sum s_{i}$, and we obtain

$$
\begin{aligned}
\Gamma\left(1-b_{0}\right) \int_{\sigma_{0}(\Delta)} \omega & =c \int_{0}^{\infty} \ldots \int_{0}^{\infty} e^{-s_{1}-\cdots-s_{n+1}} s_{1}^{b_{1}} \ldots s_{n+1}^{1+b_{n+1}} \frac{d s_{1}}{s_{1}} \wedge \ldots \wedge \frac{d s_{n+1}}{s_{n+1}} \\
& =c \Gamma\left(b_{1}\right) \Gamma\left(b_{2}\right) \ldots \Gamma\left(b_{n}\right) \Gamma\left(1+b_{n+1}\right) \\
& =c b_{n+1} \Gamma\left(b_{1}\right) \ldots \Gamma\left(b_{n+1}\right) .
\end{aligned}
$$

The formula recalled above shows that

$$
\Gamma\left(1-b_{0}\right)=\pi /\left(\sin \pi b_{0}\right) \Gamma\left(b_{0}\right),
$$

and so

$$
\begin{aligned}
c \Gamma\left(1-b_{0}\right)^{-1} & =\varepsilon^{-a_{0}} \frac{\sin \pi b_{0}}{\pi} \Gamma\left(b_{0}\right) \quad \bmod \mathbb{Q}^{\times} \\
& =\frac{1}{\pi} e^{-2 \pi i b_{0} / 2}\left(\frac{e^{\pi i b_{0}}-e^{-\pi i b_{0}}}{2 i}\right) \Gamma\left(b_{0}\right) \\
& =\frac{1}{2 \pi i}\left(1-\varepsilon^{-2 a_{0}}\right) \Gamma\left(b_{0}\right) .
\end{aligned}
$$

The lemma is now obvious.

The group algebra $\mathbb{Q}[S]$ acts on the $\mathbb{Q}$-space of differentiable $n$-simplices in $V(\mathbb{C})$. For $\mathbf{a} \in X(S)$ and $\underline{\xi}_{i}=(1, \ldots, \xi, \ldots)\left(\xi=e^{2 \pi i / d}\right.$ in the $i$ th position $)$, define

$$
\sigma=\prod_{i=0}^{n+1}\left(1-\underline{\xi}_{i}\right)^{-1} \sigma_{0}(\Delta) \subset V^{o}(\mathbb{C})
$$

where $\sigma_{0}$ and $\Delta$ are as above.

Proposition 7.13. Let $\mathbf{a} \in X(S)$ be such that no $a_{i}$ is zero, and let $\omega^{o}$ be the differential

$$
Y_{1}^{a_{1}^{\prime}} \ldots Y_{n+1}^{a_{n+1}^{\prime}} \frac{d Y_{1}}{Y_{1}} \wedge \ldots \wedge \frac{d Y_{n}}{Y_{n}}
$$

on $V^{o}$, where $a_{i}^{\prime}$ represents $-a_{i}$, and $a_{i}^{\prime} \gg 0$. Then

(a) $\underline{\xi} \omega^{o}=\underline{\xi}^{\mathbf{a}} \omega^{o}$;

(b) $\int_{\sigma} \omega^{o}=\frac{1}{2 \pi i} \prod_{i=0}^{n+1}\left(1-\xi^{a_{i}}\right) \Gamma\left(\frac{-a_{i}}{d}\right)$. 
Proof. (a) This is obvious since

$$
\underline{\zeta} Y_{i}=\left(\frac{\zeta_{i}}{\zeta_{0}}\right)^{-1} Y_{i}
$$

(b)

$$
\begin{aligned}
\int_{\sigma} \omega^{o} & =\int_{\sigma_{0}(\Delta)} \prod_{i=1}^{n+1}\left(1-\xi_{i}\right) \omega^{o} \\
& =\prod_{i=1}^{n+1}\left(1-\xi^{a_{i}}\right) \int_{\sigma_{0}(\Delta)} \omega^{o} \\
& =\frac{1}{2 \pi i} \prod_{i=0}^{n+1}\left(1-\xi^{a_{i}}\right) \Gamma\left(-\frac{a_{i}}{d}\right) .
\end{aligned}
$$

REMARK 7.14. From the Gysin sequence

$$
(\mathbb{C} \approx) \quad H^{n-2}\left(V \backslash V^{o}, \mathbb{C}\right) \rightarrow H^{n}(V, \mathbb{C}) \rightarrow H^{n}\left(V^{o}, \mathbb{C}\right) \rightarrow 0
$$

we obtain an isomorphism

$$
H^{n}(V, \mathbb{C})_{\text {prim }} \rightarrow H^{n}\left(V^{o}, \mathbb{C}\right),
$$

which shows that there is an isomorphism

$$
H_{\mathrm{dR}}^{n}(V / k)_{\text {prim }} \rightarrow H_{\mathrm{dR}}^{n}\left(V^{o} / k\right)=\Gamma\left(V^{o}, \Omega^{n}\right) / d \Gamma\left(V^{o}, \Omega^{n+1}\right) .
$$

The class $\left[\omega^{o}\right]$ of the differential $\omega^{o}$ lies in $H_{\mathrm{dR}}^{n}(V / k)_{\mathbf{a}}$. Correspondingly, we get a $C^{\infty}$ differential $n$-form on $V(\mathbb{C})$ such that

(a) the class $[\omega]$ of $\omega$ in $H_{\mathrm{dR}}^{n}(V / \mathbb{C})$ lies in $H_{\mathrm{dR}}^{n}(V / k)_{\mathbf{a}}$, and

(b) $\int_{\sigma} \omega=\frac{1}{2 \pi i} \prod_{i=0}^{n+1}\left(1-\xi^{a_{i}}\right) \Gamma\left(-\frac{a_{i}}{d}\right)$, where $\sigma=\prod_{i=1}^{n+1}\left(1-\underline{\xi}_{i}\right)^{-1} \sigma_{0}(\Delta)$.

Note that, if we regard $V$ as a variety over $\mathbb{Q}$, then $[\omega]$ even lies in $H_{\mathrm{dR}}^{n}(V / \mathbb{Q})$.

\section{The theorem}

Recall that for $\mathbf{a} \in X(S)$, we set

$$
\tilde{\Gamma}(\mathbf{a})=(2 \pi i)^{-\langle\mathbf{a}\rangle} \prod_{i=0}^{n+1} \Gamma\left(\frac{a_{i}}{d}\right) \quad\left(\in \mathbb{C} / \mathbb{Q}^{\times}\right)
$$

and for $\mathfrak{p}$ a prime ideal of $k$ not dividing $d$, we set

$$
\begin{gathered}
g(\mathfrak{p}, \mathbf{a})=q^{-\langle\mathbf{a}\rangle} \prod_{i=0}^{n+1} g\left(\mathfrak{p}, a_{i}, \psi\right) \\
g\left(\mathfrak{p}, a_{i}, \psi\right)=-\sum_{x \in \mathbb{F}_{q}} t\left(x^{\frac{1-q}{d}}\right)^{a_{i}} \psi(x)
\end{gathered}
$$

where $q$ is the order of the residue field of $\mathfrak{p}$. 
THEOREM 7.15. Let $\mathbf{a} \in X(S)$ have no $a_{i}=0$ and be such that $\langle u \mathbf{a}\rangle=\langle\mathbf{a}\rangle \quad(=n / 2+1)$ for all $u \in(\mathbb{Z} / d \mathbb{Z})^{x}$.

(a) Then $\tilde{\Gamma}(\mathbf{a}) \in \overline{\mathbb{Q}}$ and generates an abelian extension of $k \stackrel{\text { def }}{=} \mathbb{Q}\left(e^{\frac{2 \pi i}{d}}\right)$.

(b) If $F_{\mathfrak{p}} \in \mathrm{Gal}(\overline{\mathbb{Q}} / k)^{\mathrm{ab}}$ is the geometric Frobenius element at $\mathfrak{p}$, then

$$
F_{\mathfrak{p}}(\tilde{\Gamma}(\mathbf{a}))=g(\mathfrak{p}, \mathbf{a}) \tilde{\Gamma}(\mathbf{a}) .
$$

(c) For all $\tau \in \operatorname{Gal}(\overline{\mathbb{Q}} / \mathbb{Q}), \lambda_{\mathbf{a}}(\tau) \stackrel{\text { def }}{=} \tilde{\Gamma}(\mathbf{a}) / \tau \tilde{\Gamma}(\mathbf{a})$ lies in $k$; moreover, for all $u \in(\mathbb{Z} / d \mathbb{Z})^{\times}$,

$$
\tau_{u}\left(\lambda_{\mathbf{a}}(\tau)\right)=\lambda_{u \mathbf{a}}(\tau)
$$

where $\tau_{u}$ is the element of $\operatorname{Gal}(k / \mathbb{Q})$ defined by $u$.

Proof. Choose $\sigma \in H_{n}^{B}(V)$ and $\omega$ as in (7.14). Then all the conditions of (7.2) are fulfilled with $\alpha$ the character a. Moreover, (7.14) and (7.11) show respectively that

$$
P(\sigma, \omega)=\xi(\mathbf{a}) \tilde{\Gamma}(-\mathbf{a}), \quad \text { where } \xi(\mathbf{a})=\prod_{i=0}^{n+1}\left(1-\xi^{a_{i}}\right),
$$

and

$$
\chi\left(F_{\mathfrak{p}}\right)=g(\mathfrak{p}, \mathbf{a})^{-1} .
$$

As $\xi(\mathbf{a}) \in k,(7.2)$ shows that $\tilde{\Gamma}(-\mathbf{a})$ generates an abelian algebraic extension of $k$ and that

$$
F_{\mathfrak{p}} \tilde{\Gamma}(-\mathbf{a})=g(\mathfrak{p}, \mathbf{a})^{-1} \tilde{\Gamma}(-\mathbf{a}) .
$$

It is clear from this equation that $g(\mathfrak{p}, \mathbf{a})$ has absolute value 1 (in fact, it is a root of 1 ); thus

$$
g(\mathfrak{p}, \mathbf{a})^{-1}=\overline{g(\mathfrak{p}, \mathbf{a})}=g(\mathfrak{p},-\mathbf{a}) .
$$

This proves (a) and (b) for $-\mathbf{a}$ and hence for $\mathbf{a}$.

To prove (c) we have to regard $V$ as a variety over $\mathbb{Q}$. If $S$ is interpreted as an algebraic group, then its action on $V$ is rational over $\mathbb{Q}$. This means that

$$
\tau(\underline{\zeta \mathbf{x}})=\tau(\underline{\zeta}) \tau(\mathbf{x}), \quad \tau \in \operatorname{Gal}(\overline{\mathbb{Q}} / \mathbb{Q}), \quad \underline{\zeta} \in S(\overline{\mathbb{Q}}), \quad \mathbf{x} \in V(\overline{\mathbb{Q}})
$$

and implies that

$$
\tau(\underline{\zeta \gamma})=\tau(\underline{\zeta}) \tau(\gamma), \quad \tau \in \operatorname{Gal}(\overline{\mathbb{Q}} / \mathbb{Q}), \quad \underline{\zeta} \in S(\overline{\mathbb{Q}}), \quad \gamma \in C_{\mathrm{AH}}^{n}(\bar{V}) .
$$

Therefore $\operatorname{Gal}(\overline{\mathbb{Q}} / \mathbb{Q})$ stabilizes $C_{\mathrm{AH}}^{n}(\bar{V})_{[\mathrm{a}]}$ and, as this is a one-dimensional vector space over $\mathbb{Q}[\mathbf{a}]$, there exists for any $\gamma \in C_{\mathrm{AH}}^{n}(\bar{V})_{[\mathbf{a}]}$ a crossed homomorphism $\lambda: \operatorname{Gal}(\overline{\mathbb{Q}} / \mathbb{Q}) \rightarrow$ $\mathbb{Q}[\mathbf{a}]^{\times}$such that $\tau(\gamma)=\lambda(\tau) \gamma$ for all $\tau$. On applying the canonical map $C_{\mathrm{AH}}^{n}(\bar{V})_{[\mathbf{a}]} \rightarrow$ $\left(C_{\mathrm{AH}}^{n}(\bar{V}) \otimes k\right)_{[\mathrm{a}]}$ to this equality, we obtain

$$
\tau(\gamma \otimes 1)=\lambda(\tau)^{\mathbf{a}}(\gamma \otimes 1) .
$$

We take $\gamma$ to be the image of $\sigma \otimes(2 \pi i)^{-n / 2} \in H_{n}^{B}(V)\left(-\frac{n}{2}\right)$ in $C_{\mathrm{AH}}^{n}(\bar{V})_{[\mathrm{a}]}$. Then (cf. 7.3), $(\gamma \otimes 1)_{\mathrm{dR}}=P(\sigma, \omega)^{-1}[\omega]$ if $[\omega]$ is as in (7.14). Hence

$$
\lambda(\tau)^{\mathbf{a}}=\frac{P(\sigma, \omega)}{\tau P(\sigma, \omega)}=\lambda_{-\mathbf{a}}(\tau) \frac{\xi(\mathbf{a})}{\tau \xi(\mathbf{a})} .
$$


On comparing

$$
\begin{aligned}
\lambda_{\mathbf{a}}(\tau) & =\lambda(\tau)^{-\mathbf{a}} \frac{\tau \xi(-\mathbf{a})}{\xi(-\mathbf{a})} \quad \text { and } \\
\lambda_{u \mathbf{a}}(\tau) & =\lambda(\tau)^{-u \mathbf{a}} \frac{\tau \xi(-u \mathbf{a})}{\xi(-u \mathbf{a})},
\end{aligned}
$$

and using that

$$
\tau(\xi(-u \mathbf{a}))=\tau\left(\tau_{u}(\xi(-\mathbf{a}))\right)=\tau_{u}(\tau \xi(-\mathbf{a})),
$$

one obtains (c) of the theorem.

REMARK 7.16. (a) The first statement of the theorem, that $\tilde{\Gamma}$ (a) is algebraic, has an elementary proof; see the appendix by Koblitz and Ogus to Deligne 1979a.

(b) Part (b) of the theorem has been proved up to sign by Gross and Koblitz $(1979,4.5)$ using $p$-adic methods.

REMARK 7.17. Let $I_{d}$ be the group of ideals of $k$ prime to $d$, and consider the character

$$
\mathfrak{a}=\prod \mathfrak{p}_{i}^{r_{i}} \mapsto g(\mathfrak{a}, \mathbf{a}) \stackrel{\text { def }}{=} \prod g\left(\mathfrak{p}_{i}, \mathbf{a}\right)^{r_{i}}: I_{d} \rightarrow k^{\times} .
$$

When a satisfies the conditions of the theorem, then this is an algebraic Hecke character (Weil 1952, 1974; see also Deligne 1972, §6). This means that there exists an ideal $\mathfrak{m}$ of $k$ (dividing a power of $d$ ) and a homomorphism $\chi_{\text {alg }}: k^{\times} \rightarrow k^{\times}$that is algebraic (i.e., defined by a map of tori) and such that, for all $x \in k^{\times}$totally positive and $\equiv 1 \bmod \mathfrak{m}$, $g((x), \mathbf{a})=\chi_{\mathrm{alg}}(x)$. There is then a unique character

$$
\chi_{\mathbf{a}}: \operatorname{Gal}(\overline{\mathbb{Q}} / k)^{\mathrm{ab}} \rightarrow k^{\times}
$$

such that $\chi_{\mathbf{a}}\left(F_{\mathfrak{p}}\right)=g(\mathfrak{p}, \mathbf{a})$ for all $\mathfrak{p}$ prime to $d$. Part $(\mathrm{b})$ of the theorem can be stated as

$$
\sigma(\tilde{\Gamma}(\mathbf{a}))=\chi_{\mathbf{a}}(\sigma) \tilde{\Gamma}(\mathbf{a}), \text { all } \sigma \in \operatorname{Gal}(\bar{k} / k) .
$$

(There is an elegant treatment of algebraic Hecke characters in Serre 1968, Chapter II. Such a character with conductor dividing a modulus $\mathfrak{m}$ corresponds to a character $\chi$ of the torus $S_{\mathfrak{m}}$ (ibid. p. II-17). The map $\chi_{\text {alg }}$ is

$$
k^{\times} \stackrel{\pi}{\rightarrow} T_{\mathfrak{m}} \hookrightarrow S_{\mathfrak{m}} \stackrel{\chi}{\rightarrow} k^{\times} .
$$

One defines from $\chi$ a character $\chi_{\infty}$ of the idèle class group as in (ibid. II 2.7). Weil's determination of $\chi_{\text {alg }}$ shows that $\chi_{\infty}$ is of finite order; in particular, it is trivial on the connected component of the idèle class group, and so gives rise to a character $\chi_{\mathrm{a}}: \operatorname{Gal}(\overline{\mathbb{Q}} / k)^{\mathrm{ab}} \rightarrow k^{\times}$.)

\section{Restatement of the theorem}

For $b \in d^{-1} \mathbb{Z} / \mathbb{Z}$, we write $\langle b\rangle$ for the representative of $b$ in $d^{-1} \mathbb{Z}$ with $\frac{1}{d} \leq\langle b\rangle \leq 1$. Let $\mathbf{b}=\sum n(b) \delta_{b}$ be an element of the free abelian group generated by the set $d^{-1} \mathbb{Z} / \mathbb{Z} \backslash\{0\}$, and assume that $\sum n(b)\langle u b\rangle=c$ is an integer independent of $u \in \mathbb{Z} / d \mathbb{Z}$. Define

$$
\tilde{\Gamma}(\mathbf{b})=\frac{1}{(2 \pi i)^{c}} \prod_{b} \Gamma(\langle b\rangle)^{n(b)} .
$$


Let $\mathfrak{p}$ be a prime ideal of $k$, not dividing $d$, and let $\mathbb{F}_{q}$ be the residue field at $\mathfrak{p}$. For $\psi$ a non-trivial additive character of $\mathbb{F}_{q}$, define

$$
g(\mathfrak{p}, \mathbf{b})=\frac{1}{q^{c}} \prod_{b} g(\mathfrak{p}, b, \psi)^{n(b)}, \text { where } g(\mathfrak{p}, b, \psi)=-\sum_{x \in \mathbb{F}_{q}} t\left(x^{b(1-q)}\right) \psi(x) .
$$

As in (7.17), $\mathfrak{p} \mapsto g(\mathfrak{p}, \mathbf{b})$ defines an algebraic Hecke character of $k$ and a character $\chi_{\mathbf{b}}: \operatorname{Gal}(\overline{\mathbb{Q}} / \mathbb{Q}) \rightarrow \mathbb{C}^{\times}$such that $\chi_{\mathbf{b}}\left(F_{\mathfrak{p}}\right)=g(\mathfrak{p}, \mathbf{b})$ for all $\mathfrak{p} \nmid b$.

THEOREM 7.18. Assume that $\mathbf{b} \stackrel{\text { def }}{=} \sum n(b) \delta_{b}$ satisfies the condition above.

(a) Then $\tilde{\Gamma}(\mathbf{b}) \in k^{\mathrm{ab}}$, and for all $\sigma \in \mathrm{Gal}(\overline{\mathbb{Q}} / k)^{\mathrm{ab}}$,

$$
\sigma \tilde{\Gamma}(\mathbf{b})=\chi_{\mathbf{b}}(\sigma) \tilde{\Gamma}(\mathbf{b})
$$

(b) For $\tau \in \operatorname{Gal}(\overline{\mathbb{Q}} / \mathbb{Q})$, let $\lambda_{\mathbf{b}}(\tau)=\tilde{\Gamma}(\mathbf{b}) / \tau \tilde{\Gamma}(\mathbf{b})$; then $\lambda_{\mathbf{b}}(\tau) \in k$, and, for any $u \in$ $(\mathbb{Z} / d \mathbb{Z})^{\times}$,

$$
\tau_{u}\left(\lambda_{\mathbf{b}}(\tau)\right)=\lambda_{u \mathbf{b}}(\tau)
$$

Proof. Suppose first that $n(b) \geq 0$ for all $b$. Let $n+2=\sum n(b)$, and let a be an $(n+2)$ tuple in which each $a \in \mathbb{Z} / d \mathbb{Z}$ occurs exactly $n \frac{a}{d}$ times. Write $\mathbf{a}=\left(a_{0}, \ldots, a_{n+1}\right)$. Then

$$
\sum a_{i}=d\left(\sum n(b) b\right)=d c \quad(\bmod d)=0,
$$

and so $\mathbf{a} \in X(S)$. Moreover,

$$
\langle u \mathbf{a}\rangle \stackrel{\text { def }}{=} \frac{1}{d} \sum\left\langle u a_{i}\right\rangle=\sum n(b)\langle u b\rangle=c
$$

for all $u \in \mathbb{Z} / d \mathbb{Z}$. Thus $\langle u \mathbf{a}\rangle$ is constant, and $c=\langle\mathbf{a}\rangle$. We deduce that $\tilde{\Gamma}(\mathbf{a})=\tilde{\Gamma}(\mathbf{b})$, $g(\mathfrak{p}, \mathbf{a})=g(\mathfrak{p}, \mathbf{b})$, and $\chi_{\mathbf{a}}=\chi_{\mathbf{b}}$. Thus, in this case, (7.18) follows immediately from (7.15) and (7.17).

Let $\mathbf{b}$ be arbitrary. For some $N, \mathbf{b}+N \mathbf{b}_{0}$ has positive coefficients, where $\mathbf{b}_{0}=\sum \delta_{b}$. Thus (7.18) is true for $\mathbf{b}+N \mathbf{b}_{0}$. Since

$$
\tilde{\Gamma}\left(\mathbf{b}_{1}+\mathbf{b}_{2}\right)=\tilde{\Gamma}\left(\mathbf{b}_{1}\right) \tilde{\Gamma}\left(\mathbf{b}_{2}\right) \quad \bmod \mathbb{Q}^{\times}
$$

and

$$
g\left(\mathfrak{p}, \mathbf{b}_{1}+\mathbf{b}_{2}\right)=g\left(\mathfrak{p}, \mathbf{b}_{1}\right) g\left(\mathfrak{p}, \mathbf{b}_{2}\right)
$$

this completes the proof.

REMARK 7.19. (a) Part (b) of the theorem determines $\Gamma(u \mathbf{b})$ (up to multiplication by a rational number) starting from $\Gamma(\mathbf{b})$.

(b) Conjecture 8.11 of Deligne 1979a is a special case of part (a) of the above theorem. The more precise form of the conjecture, ibid. 8.13, can be proved by a modification of the above methods. 


\section{FINAL NOTE}

The original seminar of Deligne comprised fifteen lectures, given between 29/10/78 and $15 / 5 / 79$. The first six sections of these notes are based on the first eight lectures of the seminar, and the seventh section on the last two lectures. The remaining five lectures, which the writer of these notes was unable to attend, were on the following topics:

6/3/79 review of the proof that Hodge cycles on abelian varieties are absolutely Hodge; discussion of the expected action of the Frobenius endomorphism on the image of an absolute Hodge cycle in crystalline cohomology;

13/3/79 definition of the category of motives using absolute Hodge cycles; semisimplicity of the category; existence of the motivic Galois group $G$;

20/3/79 fibre functors in terms of torsors; the motives of Fermat hypersurfaces and $K 3$ surfaces are contained in the category generated by abelian varieties;

27/3/79 Artin motives; the exact sequence

$$
1 \rightarrow G^{\circ} \rightarrow G \stackrel{\pi}{\rightarrow} \operatorname{Gal}(\overline{\mathbb{Q}} / \mathbb{Q}) \rightarrow 1
$$

indentification of $G^{\circ}$ with the Serre group, and description of the $G^{\circ}$-torsor $\pi^{-1}(\tau)$; $3 / 4 / 79$ action of $\operatorname{Gal}(\overline{\mathbb{Q}} / \mathbb{Q})$ on $G^{\circ}$; study of $G \otimes_{\mathbb{Q}} \mathbb{Q}_{\ell} ;$ Hasse principle for $H^{1}\left(\mathbb{Q}, G^{\circ}\right)$.

Most of the material in these five lectures is contained in Deligne and Milne 1982, §6, and Deligne 1982.

The writer of these notes is indebted to P. Deligne and A. Ogus for their criticisms of the first draft of the notes and to Ogus for his notes on which section seven is largely based.

\section{References}

Artin, M., Grothendieck, A., And Verdier, J. 1972, 1973. Théorie des topos et cohomologie étale des schémas. Lecture Notes in Math. 269, 270, 305. Springer-Verlag, Berlin. SGA4.

BAILY, JR., W. L. AND BOREL, A. 1966. Compactification of arithmetic quotients of bounded symmetric domains. Ann. of Math. (2) 84:442-528.

Borel, A. 1972. Some metric properties of arithmetic quotients of symmetric spaces and an extension theorem. J. Differential Geometry 6:543-560.

Borel, A. AND Springer, T. A. 1966. Rationality properties of linear algebraic groups, pp. 26-32. In Algebraic Groups and Discontinuous Subgroups (Proc. Sympos. Pure Math., Boulder, Colo., 1965). Amer. Math. Soc., Providence, R.I.

Borovoi, M. V. 1977. The Shimura-Deligne schemes $M_{\mathbf{C}}(G, h)$ and the rational cohomology classes of type $(p, p)$ of abelian varieties, pp. 3-53. In Problems of group theory and homological algebra, No. 1 (Russian). Yaroslav. Gos. Univ., Yaroslavl'.

Deligne, P. 1968. Théorème de Lefschetz et critères de dégénérescence de suites spectrales. Inst. Hautes Études Sci. Publ. Math. 35:259-278.

Deligne, P. 1971a. Théorie de Hodge. II. Inst. Hautes Études Sci. Publ. Math. 40:5-57.

Deligne, P. 1971b. Travaux de Griffiths, pp. Lecture Notes in Math., Vol. 180. In Séminaire Bourbaki, 22ème année (1969/70), Exp. No. 376. Springer, Berlin. 
Deligne, P. 1971c. Travaux de Shimura, pp. 123-165. Lecture Notes in Math., Vol. 244. In Séminaire Bourbaki, 23ème année (1970/71), Exp. No. 389. Springer, Berlin.

Deligne, P. 1972. La conjecture de Weil pour les surfaces K3. Invent. Math. 15:206-226.

Deligne, P. 1979a. Valeurs de fonctions $L$ et périodes d'intégrales, pp. 313-346. In Automorphic forms, representations and $L$-functions (Proc. Sympos. Pure Math., Oregon State Univ., Corvallis, Ore., 1977), Part 2, Proc. Sympos. Pure Math., XXXIII. Amer. Math. Soc., Providence, R.I.

Deligne, P. 1979b. Variétés de Shimura: interprétation modulaire, et techniques de construction de modèles canoniques, pp. 247-289. In Automorphic forms, representations and $L$-functions (Proc. Sympos. Pure Math., Oregon State Univ., Corvallis, Ore., 1977), Part 2, Proc. Sympos. Pure Math., XXXIII. Amer. Math. Soc., Providence, R.I.

Deligne, P. 1982. Motifs et groupes de Taniyama, pp. 261-279. In Hodge cycles, motives, and Shimura varieties, volume 900 of Lecture Notes in Mathematics. Springer-Verlag, Berlin-New York.

Deligne, P. And Milne, J. S. 1982. Tannakian categories, pp. 101-228. In Hodge cycles, motives, and Shimura varieties, Lecture Notes in Mathematics 900. Springer-Verlag, Berlin.

Deligne, P., Milne, J. S., Ogus, A., And Shih, K.-Y. 1982. Hodge cycles, motives, and Shimura varieties, volume 900 of Lecture Notes in Mathematics 900. Springer-Verlag, Berlin.

Demazure, M. 1976. Démonstration de la conjecture de Mumford (d'après W. Haboush), pp. 138-144. Lecture Notes in Math., Vol. 514. In Séminaire Bourbaki (1974/1975: Exposés Nos. 453-470), Exp. No. 462. Springer, Berlin.

GRIFFITHS, P. A. 1969. On the periods of certain rational integrals. I, II. Ann. of Math. (2) 90 (1969), 460-495; ibid. (2) 90:496-541.

GROSS, B. H. 1978. On the periods of abelian integrals and a formula of Chowla and Selberg. Invent. Math. 45:193-211.

Gross, B. H. And Koblitz, N. 1979. Gauss sums and the $p$-adic $\Gamma$-function. Ann. of Math. (2) 109:569-581.

Grothendieck, A. 1958. La théorie des classes de Chern. Bull. Soc. Math. France 86:137-154.

Grothendieck, A. 1966. On the de Rham cohomology of algebraic varieties. Inst. Hautes Études Sci. Publ. Math. 29:95-103.

KATZ, N. M. 1970. Nilpotent connections and the monodromy theorem: Applications of a result of Turrittin. Inst. Hautes Études Sci. Publ. Math. 39:175-232.

KATZ, N. M. AND ODA, T. 1968. On the differentiation of de Rham cohomology classes with respect to parameters. J. Math. Kyoto Univ. 8:199-213.

KNAPP, A. W. 1972. Bounded symmetric domains and holomorphic discrete series, pp. 211-246. Pure and Appl. Math., Vol. 8. In Symmetric spaces (Short Courses, Washington Univ., St. Louis, Mo., 1969-1970). Dekker, New York.

LANDHERR, W. 1936. Aquivalenz Hermitescher Formen über einem beliebigen algebraischen Zahlkörper. Abh. Math. Semin. Hamb. Univ. 11:245-248.

Milne, J. S. 1980. Etale cohomology, volume 33 of Princeton Mathematical Series. Princeton University Press, Princeton, N.J. 
Milne, J. S. And ShiH, K.-Y. 1982. Conjugates of Shimura varieties, pp. 280-356. In Hodge cycles, motives, and Shimura varieties, Lecture Notes in Mathematics. Springer-Verlag, Berlin.

PJATECKII $\breve{S}$ APIRO, I. I. 1971. Interrelations between the Tate and Hodge hypotheses for abelian varieties. Mat. Sb. (N.S.) 85(127):610-620.

Pohlmann, H. 1968. Algebraic cycles on abelian varieties of complex multiplication type. Ann. of Math. (2) 88:161-180.

SaAVedra Rivano, N. 1972. Catégories Tannakiennes. Lecture Notes in Mathematics, Vol. 265. Springer-Verlag, Berlin.

SERRE, J.-P. 1955-1956. Géométrie algébrique et géométrie analytique. Ann. Inst. Fourier, Grenoble 6:1-42.

SERRE, J.-P. 1968. Abelian $l$-adic representations and elliptic curves. McGill University lecture notes written with the collaboration of Willem Kuyk and John Labute. W. A. Benjamin, Inc., New York-Amsterdam.

Singer, I. M. AND Thorpe, J. A. 1967. Lecture notes on elementary topology and geometry. Scott, Foresman and Co., Glenview, Ill.

WARnER, F. W. 1971. Foundations of differentiable manifolds and Lie groups. Scott, Foresman and Co., Glenview, Ill.-London.

WATERhouse, W. C. 1979. Introduction to affine group schemes, volume 66 of Graduate Texts in Mathematics. Springer-Verlag, New York.

WEIL, A. 1952. Jacobi sums as "Grössencharaktere". Trans. Amer. Math. Soc. 73:487-495.

WEIL, A. 1958. Introduction à l'étude des variétés kählériennes. Publications de l'Institut de Mathématique de l'Université de Nancago, VI. Actualités Sci. Ind. no. 1267. Hermann, Paris.

WeIL, A. 1974. Sommes de Jacobi et caractères de Hecke. Nachr. Akad. Wiss. Göttingen Math.-Phys. Kl. II pp. 1-14. 


\section{Endnotes (by J.S. Milne)}

M.1. (p. 1) The following changes from the original have been made:

$\diamond$ Numerous minor improvements to the exposition.

$\diamond \quad$ Numerous misprints fixed; major corrections have been noted in these endnotes.

$\diamond$ Part of the general introduction to the volume Deligne et al. 1982 (LNM 900) has been placed at the start of the introduction.

$\diamond$ Some changes of notation have been made - the footnote $\mathrm{DR}_{\mathrm{D}}$ has been replaced by $\mathrm{dR}$, $\mathbb{A}^{f}$ has been replaced by $\mathbb{A}_{f}$, and $\approx$ (isomorphism) has been distinguished from $\simeq$ (canonical isomorphism).

$\diamond \quad$ These endnotes and all footnotes marked "Added" have been added.

$\diamond$ The original numbering has been retained.

M.2. (p. 3) For a description of these consequences, see

Deligne, Pierre, Cycles de Hodge absolus et périodes des intégrales des variétés abéliennes. Abelian functions and transcendental numbers (Colloq., École Polytech., Palaiseau, 1979). Mém. Soc. Math. France (N.S.) 1980/81, no. 2, 23-33.

For applications of the results of these notes to the periods of motives attached to Hecke characters, see

Schappacher, Norbert, Periods of Hecke characters. Lecture Notes in Mathematics, 1301. Springer-Verlag, Berlin, 1988.

M.3. (p. 4) Say that a cohomology class in $H^{2 p}(A, \mathbb{Q})(p)$ is a split Weil class if there exists

$\diamond \quad$ a CM-field $E$,

$\diamond \quad$ a homomorphism $v: E \rightarrow \operatorname{End}(A)$, and

$\diamond \quad$ a polarization $\theta$ of $A$ satisfying the conditions (a,b) of (4.8)

such that the class lies in the subspace $\bigwedge_{E}^{2 p} H^{1}(A, \mathbb{Q})(p)$ of $H^{2 p}(A, \mathbb{Q})(p)$.

By assumption, the algebraic classes are accessible. The proof of Theorem 4.8 will show that all split Weil classes are accessible once we check that the family in the proof contains an abelian variety for which the Hodge conjecture is true. But, in the proof, we can take $A_{0}$ to be any abelian variety of dimension $d / 2$, and it is well-known that the Hodge conjecture holds for powers of an elliptic curve (see p. 107 of Tate, Algebraic cycles and poles of zeta functions, 1965). Now the argument in $\$ 5$ shows that all Hodge classes on abelian varieties of CM-type are accessible, and Proposition 6.1 shows the same result for all abelian varieties.

Condition (a) is checked in (2.1), (b) is obvious from the definition of absolute Hodge cycle, (c) is proved in (3.8), and (d) is proved in (2.12).

M.4. (p. 7) The "and so" is misleading since the degeneration of the spectral sequence by itself does not provide the Hodge decomposition. See mathoverflow questions 28265 and 311700 for a discussion of this.

From the spectral sequence, we get a descending filtration $F^{p}$ on the groups $H^{n}(X, \mathbb{C})$ such that

$$
H^{n}=\left(H^{n} \cap F^{p}\right) \oplus\left(H^{n} \cap \overline{F^{q}}\right)
$$


for all $n, p, q$ with $p+q=n+1$. This implies that

$$
H^{n}=\oplus H^{p, q}
$$

with

$$
H^{p, q}=H^{p+q} \cap F^{p} \cap \overline{F^{q}}=H^{q}\left(X, \Omega^{p}\right) .
$$

M.5. (p. 14) Grothendieck conjectured that the only relations between the periods come from algebaic cycles.

$\ldots$ it is believed that if [the elliptic curve] is algebraic (i.e., its coefficients $g_{2}$ and $g_{3}$ are algebraic), then $\omega_{2}$ and $\omega_{3}$ are transcendental, and it is believed that if $X$ has no complex multiplication, then $\omega_{1}$ and $\omega_{2}$ are algebraically independent. This conjecture extends in an obvious way to the set of periods $\left(\omega_{1}, \omega_{2}, \eta_{1}, \eta_{2}\right)$ and can be rephrased also for curves of any genus, or rather for abelian varieties of dimension $g$, involving $4 g$ periods. (Grothendieck 1966, p102).

Also:

For the period matrix itself, Grothendieck has made a very interesting conjecture concerning its relations, and his conjecture applies to a general situation as follows. Let $V$ be a projective, nonsingular variety defined over the rational numbers. One can define the cohomology of $V$ with rational coefficients in two ways. First, by means of differential forms (de Rham), purely algebraically, thereby obtaining a vector space $H_{\text {diff }}(V, \mathbb{Q})$ over $\mathbb{Q}$. Secondly, one can take the singular cohomology $H_{\text {sing }}(V, \mathbb{Q})$ with rational coefficients, i.e., the singular cohomology of the complex manifold $V_{\mathbb{C}}$. Let us select a basis for each of these vector spaces over $\mathbb{Q}$, and let us tensor these spaces over $\mathbb{C}$. Then there is a unique (period) matrix $\Omega$ with complex coefficients which transforms one basis into the other. Any algebraic cycle on $V$ or the products of $V$ with itself will give rise to a polynomial relation with rational coefficients among the coefficients of this matrix. Grothendieck's conjecture is that the ideal generated by these relations is an ideal of definition for the period matrix. (S. Lang, Introduction to Transcendental Numbers, Addison-Wesley, 1966, pp42-43; Collected Works, Vol. I, pp. 443-444.)

M.6. (p. 16) So far as I know, both (2.2) and (2.4) remain open.

M.7. (p. 19) (2.11) The theorem extends to one-motives (Théorème 2.2.5 of Brylinski, Jean-Luc, "1-motifs" et formes automorphes. Journées Automorphes (Dijon, 1981), 43-106, Publ. Math. Univ. Paris VII, 15, Univ. Paris VII, Paris, 1983.)

M.8. (p. 21) (2.14) By using the full strength of Deligne's results on cohomology, it is possible to avoid the use of the Gauss-Manin connection in the proof of Theorem 2.12 (Blasius, Don, A $p$-adic property of Hodge classes on abelian varieties. Motives (Seattle, WA, 1991), 293-308, Proc. Sympos. Pure Math., 55, Part 2, Amer. Math. Soc., Providence, RI, 1994, Theorem 3.1).

THEOREM (Deligne 1971a). Let $\pi: X \rightarrow S$ be a smooth proper morphism of smooth varieties over $\mathbb{C}$.

(a) The Leray spectral sequence

$$
H^{r}\left(S, R^{s} \pi_{*} \mathbb{Q}\right) \Rightarrow H^{r+s}(X, \mathbb{Q})
$$


degenerates at $E_{2}$; in particular, the edge morphism

$$
H^{n}(X, \mathbb{Q}) \rightarrow \Gamma\left(S, R^{n} \pi_{*} \mathbb{Q}\right)
$$

is surjective.

(b) If $\bar{X}$ is a smooth compactification of $X$ with $\bar{X} \backslash X$ a union of smooth divisors with normal crossings, then the canonical morphism

$$
H^{n}(\bar{X}, \mathbb{Q}) \rightarrow \Gamma\left(S, R^{n} \pi_{*} \mathbb{Q}\right)
$$

is surjective.

(c) Let $\left(R^{n} \pi_{*} \mathbb{Q}\right)^{0}$ be the largest constant local subsystem of $R^{n} \pi_{*} \mathbb{Q}\left(\operatorname{so}\left(R^{n} \pi_{*} \mathbb{Q}\right)_{s}^{0}=\right.$ $\Gamma\left(S, R^{n} \pi_{*} \mathbb{Q}\right)$ for all $\left.s \in S(\mathbb{C})\right)$. For each $s \in S,\left(R^{n} \pi_{*} \mathbb{Q}\right)_{s}^{0}$ is a Hodge substructure of $\left(R^{n} \pi_{*} \mathbb{Q}\right)_{s}=H^{n}\left(X_{s}, \mathbb{Q}\right)$, and the induced Hodge structure on $\Gamma\left(S, R^{n} \pi_{*} \mathbb{Q}\right)$ is independent of $s$.

In particular, the map

$$
H^{n}(\bar{X}, \mathbb{Q}) \rightarrow H^{n}\left(X_{s}, \mathbb{Q}\right)
$$

has image $\left(R^{n} \pi_{*} \mathbb{Q}\right)_{s}^{0}$, and its kernel is independent of $s$.

THEOREM. Let $\pi: X \rightarrow S$ be a smooth proper morphism of complex varieties with $S$ smooth and connected. Let $\gamma \in \Gamma\left(S, R^{2 n} \pi_{*} \mathbb{Q}(n)\right)$.

(a) If $\gamma_{s}$ is a Hodge cycle for one $s \in S(\mathbb{C})$, then it is a Hodge cycle for every $s \in S(\mathbb{C})$;

(b) If $\gamma_{s}$ is an absolute Hodge cycle for one $s \in S(\mathbb{C})$, then it is an absolute Hodge class for every $s \in S(\mathbb{C})$.

Proof. (Blasius 1994, 3.1.) According to Deligne's theorem, for $s, t \in S(\mathbb{C})$, there is a commutative diagram:

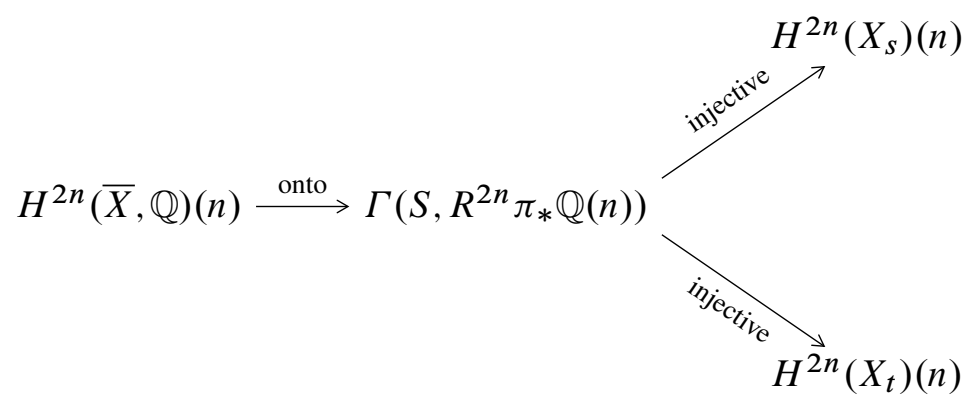

Let $\gamma \in \Gamma\left(S, R^{2 n} \pi_{*} \mathbb{Q}(n)\right)$. According to (c) of the theorem, $\Gamma\left(S, R^{2 n} \pi_{*} \mathbb{Q}(n)\right)$ has a Hodge structure for which the diagonal maps are morphisms of Hodge structures. It follows that if $\gamma_{s}$ is a Hodge cycle, then so also are $\gamma$ and $\gamma_{t}$.

Identify $H(X) \otimes \mathbb{A}$ with $H_{\mathbb{A}}(X)$. Let $\sigma$ be an automorphism of $\mathbb{C}$. If $\gamma_{s}$ is a Hodge cycle on $X_{s}$ relative to $\sigma$, then there is a $\gamma_{s}^{\sigma} \in H^{2 n}\left(\sigma X_{s}\right)(n)$ such that $\gamma_{s}^{\sigma} \otimes 1=\sigma\left(\gamma_{s} \otimes 1\right)$ in $H_{\mathbb{A}}^{2 n}\left(\sigma X_{S}\right)$. Since $\sigma\left(\gamma_{s} \otimes 1\right)$ is in the image of

$$
H^{2 n}(\sigma \bar{X})(n) \otimes \mathbb{A} \rightarrow H^{2 n}\left(\sigma X_{S}\right)(n) \otimes \mathbb{A},
$$

$\gamma_{s}^{\sigma}$ is in the image of

$$
H^{2 n}(\sigma \bar{X})(n) \rightarrow H^{2 n}\left(\sigma X_{S}\right)(n)
$$


(apply 2.13) - let $\tilde{\gamma}^{\sigma} \in H^{2 n}(\sigma \bar{X})(n)$ map to $\gamma_{s}^{\sigma}$. Because $\gamma_{s}$ and $\gamma_{t}$ have a common pre-image in $\Gamma\left(S, R^{2 n} \pi_{*} \mathbb{Q}(n)\right), \sigma\left(\gamma_{s} \otimes 1\right)$ and $\sigma\left(\gamma_{t} \otimes 1\right)$ have a common pre-image in $\Gamma\left(\sigma S, R^{2 n} \pi_{*} \mathbb{Q}(n)\right) \otimes \mathbb{A}$. Therefore (see the diagram), $\tilde{\gamma}^{\sigma} \otimes 1$ maps to $\sigma\left(\gamma_{t} \otimes 1\right)$ in $H^{2 n}\left(\sigma X_{t}\right) \otimes \mathbb{A}$, and so $\gamma_{t} \otimes 1$ is a Hodge cycle relative to $\sigma$.

M.9. (p. 26) (3.8) The motivic significance of Principle A is the following: by the usual method (e.g., Saavedra 1972, VI 4.1) we can define a category of motives using the absolute Hodge classes as correspondences; this will be a pseudo-abelian rigid tensor category, and it will be Tannakian if and only if Principle A holds for all the varieties on which the category is based.

M.10. (p. 30) (4.4) Let $E$ be a CM-field, and let $v: E \rightarrow \operatorname{End}(A)$ be a homomorphism. The pair $(A, v)$ is said to be of Weil type if $\operatorname{Tgt}_{0}(A)$ is a free $E \otimes_{\mathbb{Q}} \mathbb{C}$-module. The proposition shows the following:

If $(A, v)$ is of Weil type, then the subspace $\bigwedge_{E}^{d} H^{1}(A, \mathbb{Q})$ of $H^{d}(A, \mathbb{Q})$ consists of Hodge classes.

When $E$ is quadratic over $\mathbb{Q}$, these Hodge classes were studied by Weil (Abelian varieties and the Hodge ring, 1977c in Collected Papers, Vol. III, Springer-Verlag, pp421-429), and for this reason are called Weil classes.

A polarization of an abelian variety $(A, v)$ of Weil type is a polarization $\lambda$ of $A$ whose Rosati involution stabilizes $E$ and induces complex conjugation on it.

The special Mumford-Tate group of a general polarized abelian variety $(A, v, \lambda)$ of Weil type is $\mathrm{SU}(\phi)$ where $\phi$ is the $E$-Hermitian form on $H^{1}(A, \mathbb{Q})$ defined by the polarization. If the special Mumford-Tate group of $(A, v)$ equals $\mathrm{SU}(\phi)$, then the $\mathbb{Q}$-algebra of Hodge cycles is generated by the divisor classes and the Weil classes (but not by the divisor classes alone). When $E$ is quadratic over $\mathbb{Q}$, these statements are proved in Weil (ibid.), but the same argument works in general.

For more on Weil classes, see

Moonen, B. J. J.; Zarhin, Yu. G. Weil classes on abelian varieties. J. Reine Angew. Math. 496 (1998), 83-92.

Zarhin, Yu. G. and Moonen, B. J. J., Weil classes and Rosati involutions on complex abelian varieties. Recent progress in algebra (Taejon/Seoul, 1997), 229-236, Contemp. Math., 224, Amer. Math. Soc., Providence, RI, 1999.

In a small number of cases, the Weil classes are known to be algebraic even when they are not contained in the $\mathbb{Q}$-algebra generated by the divisor classes:

Schoen, Chad, Hodge classes on self-products of a variety with an automorphism. Compositio Math. 65 (1988), no. 1, 3-32. Addendum, ibid., 114 (1998), no. 3, 329-336.

van Geemen, Bert, An introduction to the Hodge conjecture for abelian varieties. Algebraic cycles and Hodge theory (Torino, 1993), 233-252, Lecture Notes in Math., 1594, Springer, Berlin, 1994.

M.11. (p. 31) (4.5) It is tempting to think ${ }^{7}$ that $\bigwedge_{E}^{d} H^{1}(A, \mathbb{Q})\left(\frac{d}{2}\right)$ must be the $E$-subspace of $H^{d}(A, \mathbb{Q})\left(\frac{d}{2}\right)$ spanned by the class of the algebraic cycle $A_{0}^{[E: \mathbb{Q}]-1} \times\{0\} \subset A_{0}^{[E: \mathbb{Q}]}$.

\footnotetext{
${ }^{7}$ Indeed, this is asserted in Blasius 1994, p. 305. If it had been true, it would have been possible to deduce the Hodge conjecture for Weil classes on abelian varieties from the main theorem of Bloch, Semi-regularity and de Rham cohomology, 1972 (Milne, unpublished manuscript, 1994).
} 
However, this is not true. For example, let $E$ be the subfield of $\overline{\mathbb{Q}}$ generated by $\sqrt{-n}$ and let $\sigma$ be the given embedding of $E$ into $\overline{\mathbb{Q}}$. Let $\sqrt{-n} \in E$ act on $A=A_{0}^{2}$ as $\left(\begin{array}{cc}0 & -n \\ 1 & 0\end{array}\right)$, and let $V=H^{1}\left(A_{0}, \mathbb{Q}\right)$. Then, $V \otimes E \simeq V_{\sigma} \oplus V_{\bar{\sigma}}$, and

$$
\left(\bigwedge_{E}^{d}(V \otimes E)\right) \simeq \bigwedge_{E}^{d}\left(V_{\sigma} \oplus V_{\bar{\sigma}}\right) \simeq \bigwedge_{E}^{d} V_{\sigma} \oplus \bigwedge_{E}^{d} V_{\bar{\sigma}}
$$

Let $e_{1}, \ldots, e_{d}$ be a basis for $V=H^{1}\left(A_{0}, \mathbb{Q}\right.$ ) (first copy of $A_{0}$ ), and let $f_{1}, \ldots, f_{d}$ be the same basis for the second copy. The elements $e_{i}+\sqrt{-n} f_{i}$ form a basis for $V_{\sigma}$, and so

$$
\left(e_{1}+\sqrt{-n} f_{1}\right) \wedge\left(e_{2}+\sqrt{-n} f_{2}\right) \wedge \ldots
$$

is an $E$-basis for $\bigwedge_{E}^{d} H^{1}(A, \mathbb{Q})\left(\right.$ not $\left.e_{1} \wedge e_{2} \wedge \ldots\right)$. When $d=2$, the elements

$$
e_{1} \wedge e_{2}-n f_{1} \wedge f_{2}, \quad \sqrt{-n}\left(e_{1} \otimes f_{2}+e_{2} \otimes f_{1}\right)
$$

form a $\mathbb{Q}$-basis for $\bigwedge_{E}^{2} H^{1}(A, \mathbb{Q})$, and the Weil classes are represented by the algebraic cycles $\left(0 \times A_{0}\right)-n\left(A_{0} \times 0\right)$ and the $(1,1)$-components of the diagonal. See Murty, Hodge and Weil classes on abelian varieties, 2000. It seems not to be known whether, in the situation of the lemma, the space $\bigwedge_{E}^{d} H^{1}(A, \mathbb{Q})\left(\frac{d}{2}\right)$ always consists of algebraic classes.

M.12. (p. 40) The proof shows that, in certain a Tannakian sense, the $\mathbb{Q}$-algebra of Hodge classes on an abelian variety of CM type is generated by the divisor classes and the split Weil classes. Yves André (Une remarque à propos des cycles de Hodge de type CM, 1992) proves a more precise result. As in the text, let $A$ be an abelian variety over $\mathbb{C}$ with complex multiplication by a CM-field $E$. We suppose that $E$ is Galois over $\mathbb{Q}$. Denote the inclusion $E \rightarrow \operatorname{End}^{0}(A)$ by $\nu$, and let $\phi$ be the CM-type of $(A, v)$. Then $(A, \sigma v)$ is of CM-type $\sigma \phi$. Let $J=\left\{\sigma_{1}, \ldots, \sigma_{2 r}\right\}$ be a subset of $\operatorname{Gal}(E / \mathbb{Q})$ such that $\sum \sigma_{i} \phi=r$, and let $E$ act on $A_{J} \stackrel{\text { def }}{=} A^{2 r}$ by $x \mapsto\left(\ldots,\left(\sigma_{i} \nu\right)(x), \ldots\right)$. Then the space

$$
\bigwedge_{E}^{2 r} H^{1}\left(A_{J}, \mathbb{Q}\right)(r)
$$

of Weil classes in $H^{2 r}\left(A_{J}, \mathbb{Q}(r)\right)$ consists of absolute Hodge classes (Theorem 4.8). Let $f_{J}: A \rightarrow A_{J}$ denote the diagonal map. André shows that every Hodge class on $A$ of codimension $r$ is a sum of classes of the form $f_{J}^{*}(\gamma)$ with $\gamma$ a Weil class on $A_{J}$.

M.13. (p. 42) We discuss some simplifications and applications of the proof of Theorem 2.11 .

\section{A CRITERION FOR A FAMILY OF Hodge Classes to CONTAIN ALl Hodge Classes}

THEOREM. Suppose that for each abelian variety $A$ over $\mathbb{C}$ we have a $\mathbb{Q}$-subspace $C(A)$ of the Hodge classes on A. Assume:

(a) $C(A)$ contains all algebraic classes on $A$;

(b) pull-back by a homomorphism $\alpha: A \rightarrow B$ maps $C(B)$ into $C(A)$;

(c) let $\pi: \mathcal{A} \rightarrow S$ be an abelian scheme over a connected smooth variety $S$ over $\mathbb{C}$, and let $t \in \Gamma\left(S, R^{2 p_{\pi_{*}}} \mathbb{Q}(p)\right)$; if $t_{s}$ is a Hodge cycle for all $s$ and lies in $C\left(A_{s}\right)$ for one $s$, then it lies in $C\left(A_{s}\right)$ for all $s$.

Then $C(A)$ contains all the Hodge classes on $A$.

Proof. The proof of Theorem 4.8 shows that $C(A)$ contains all split Weil classes on $A$ (see endnote M.3), and then André's improvement of $\$ 5$ (see endnote M.12) proves the theorem for all abelian varieties of CM-type. Now Proposition 6.1 completes the proof. 


\section{Algebraic Classes}

In Steenbrink (Some remarks about the Hodge conjecture. Hodge theory (Sant Cugat, 1985), 165-175, Lecture Notes in Math., 1246, Springer, Berlin, 1987) we find the following:

[Grothendieck (1966), footnote 13] stated a conjecture which is weaker than the Hodge $(p, p)$ conjecture:

(VHC) Suppose that $f: X \rightarrow S$ is a smooth projective morphism with $S$ connected, smooth. Suppose that $\lambda \in H^{0}\left(S, R^{2 p} f_{*} \mathbb{Q}_{X}\right)$ is of type $(p, p)$ everywhere, and for some $s_{0} \in S, \lambda\left(s_{0}\right)$ is the cohomology class of an algebraic cycle of codimension $p$ on $X_{s_{0}}$. Then $\lambda(s)$ is an algebraic cycle class for all $s \in S$. This "variational Hodge conjecture" ....

In fact, Grothendieck (1966, footnote 13) asks whether the following statement is true:

(VHCo) Let $S$ be a connected reduced scheme of characteristic zero, and let $\pi: X \rightarrow S$ be a proper smooth morphism; then a section $z$ of $\mathbb{R}^{2 p} \pi_{*}\left(\Omega_{X / S}^{\bullet}\right)$ is algebraic on every fibre if and only if it is horizontal for the canonical integral connection and is algebraic on one fibre.

THEOREM. If the variational Hodge conjecture (either statement (VHC) or (VHCo)) is true for abelian varieties, then so also is the Hodge conjecture.

Proof. Assume (VHC), and let $C(A)$ be the $\mathbb{Q}$-span of the classes of algebraic cycles on $A$. Then the preceding theorem immediately shows that $C(A)$ contains all Hodge classes on $A$.

The proof that (VHCo) implies the Hodge conjecture is similar, but requires the remark that all of $\S \S 2-6$ still applies when the étale component is omitted.

Although, we didn't need Principle A for the last theorem, it should be noted that it does hold for the algebraic classes on abelian varieties (those in the $\mathbb{Q}$-subspace of $H^{2 p}(A, \mathbb{Q}(p)$ ) spanned by the classes of algebraic cycles). This is a consequence of the following three results (cf. endnote M.9):

$\diamond$ numerical equivalence coincides with homological equivalence on complex abelian varieties (Lieberman, David I., Numerical and homological equivalence of algebraic cycles on Hodge manifolds. Amer. J. Math. 901968 366-374, MR37 \#5898);

$\diamond$ the category of motives defined using algebraic cycles modulo numerical equivalence is an abelian category (even semisimple) (Jannsen, Uwe, Motives, numerical equivalence, and semi-simplicity. Invent. Math. 107 (1992), no. 3, 447-452.);

$\diamond \quad$ every abelian tensor category over a field of characteristic zero whose objects have finite dimension is Tannakian (Théorème 7.1 of Deligne, P., Catégories tannakiennes. The Grothendieck Festschrift, Vol. II, 111-195, Progr. Math., 87, Birkhäuser Boston, Boston, MA, 1990).

\section{DE RHAM-Hodge CLASSES (Blasius)}

For a complete smooth variety $X$ over $\overline{\mathbb{Q}}$ and an embedding $\sigma: \overline{\mathbb{Q}} \rightarrow \overline{\mathbb{Q}}_{p}$, there is a natural isomorphism

$$
I: H_{\mathrm{et}}^{2 r}\left(\sigma X, \mathbb{Q}_{p}\right)(r) \otimes_{\mathbb{Q}_{p}} B_{\mathrm{dR}} \rightarrow H_{\mathrm{dR}}^{2 r}(\sigma X)(r) \otimes_{\overline{\mathbb{Q}}_{p}} B_{\mathrm{dR}}
$$

(Faltings, Tsuji) compatible with cycle maps. Call an absolute Hodge class $\gamma$ on $X \boldsymbol{d e}$ Rham if, for all $\sigma, I\left(\sigma \gamma_{p} \otimes 1\right)=\sigma \gamma_{\mathrm{dR}} \otimes 1$. The following is proved in Blasius 1994 . 
THEOREM. Every absolute Hodge class on an abelian variety over $\overline{\mathbb{Q}}$ is de Rham.

Proof. The functor from abelian varieties over $\overline{\mathbb{Q}}$ to abelian varieties over $\mathbb{C}$ is fully faithful and the essential image contains the abelian varieties of CM-type. Using this, one sees by the same arguments as above, that the theorem follows from the next result.

THEOREM (BLASIUS 1994, 3.1). Let $\pi: X \rightarrow S$ be a smooth proper morphism of smooth varieties over $\overline{\mathbb{Q}} \subset \mathbb{C}$ with $S$ connected, and let $\gamma \in \Gamma\left(S_{\mathbb{C}}, R^{2 n} \pi_{\mathbb{C} *} \mathbb{Q}(n)\right)$. If $\gamma_{s} \in H_{B}^{2 n}\left(X_{S}\right)(n)$ is absolutely Hodge and de Rham for one $s \in S(\overline{\mathbb{Q}})$, then it is absolutely Hodge and de Rham for every $s$.

Proof. Let $s, t \in S(\overline{\mathbb{Q}})$ and assume $\gamma_{s}$ is absolutely Hodge and de Rham. We know (see endnote M.8) that $\gamma_{t}$ is absolutely Hodge, and we have to prove it is de Rham.

Let $\sigma: \overline{\mathbb{Q}} \hookrightarrow \overline{\mathbb{Q}}_{p}$ be an embedding. For a smooth compactification $\bar{X}$ of $X$ (as in endnote M.8) over $\overline{\mathbb{Q}}$, we have a commutative diagram

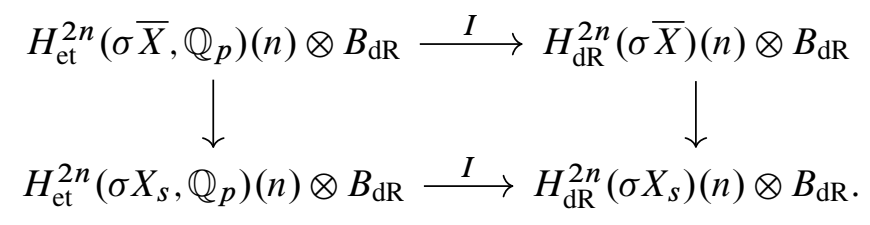

There exists $\tilde{\gamma} \in H_{B}^{2 n}(\bar{X})(n)$ mapping to $\gamma$ (see the diagram in endnote M.8). Let $\tilde{\gamma}_{p}$ and $\tilde{\gamma}_{\mathrm{dR}}$ be the images of $\tilde{\gamma}$ in $H_{\mathrm{et}}^{2 n}\left(\sigma \bar{X}, \mathbb{Q}_{p}\right)(n)$ and $H_{\mathrm{dR}}^{2 n}(\sigma \bar{X})(n)$. Because $\gamma_{s}$ is de Rham, $I\left(\tilde{\gamma}_{p} \otimes 1\right)$ differs from $\tilde{\gamma}_{\mathrm{dR}} \otimes 1$ by an element of

$$
\left(\operatorname{Ker}\left(H_{\mathrm{dR}}^{2 n}(\sigma \bar{X})(n) \rightarrow H_{\mathrm{dR}}^{2 n}\left(\sigma X_{S}\right)(n)\right) \otimes B_{\mathrm{dR}} .\right.
$$

But this kernel is independent of $s$, and so $\gamma_{t}$ is also de Rham.

\section{Motivated Classes (AbDulali, André)}

Recall that Grothendieck's Lefschetz standard conjecture says that the $\mathbb{Q}$-space of algebraic classes on a smooth algebraic variety is invariant under the Hodge $*$-operator. Abdulali (Algebraic cycles in families of abelian varieties. Canad. J. Math. 46 (1994), no. 6, 11211134 ) shows that if the $\mathbb{Q}$-spaces of algebraic cycles in the $L_{2}$-cohomology of Kuga fibre varieties (not necessarily compact) are invariant under the Hodge $*$-operator, then the Hodge conjecture is true for all abelian varieties.

André (Pour une théorie inconditionnelle des motifs, Inst. Hautes Études Sci. Publ. Math. No. 83 (1996), 5-49) proves a more precise result: every Hodge class on an abelian variety $A$ is a sum of classes of the form $p_{*}\left(\alpha \cup *_{L} \beta\right)$ in which $\alpha$ and $\beta$ are algebraic classes on a product of $A$ with an abelian variety and certain total spaces of compact pencils of abelian varieties.

In outline, the proofs are similar to that of Theorem 2.11.

M.14. (p. 43) Since Theorem 2.11 is true for one-motives (see endnote M.7), so also is the

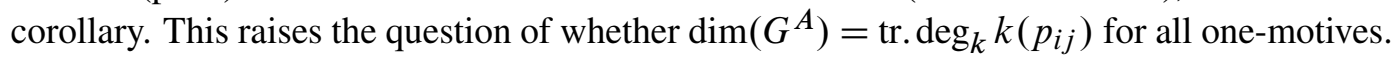
For a discussion of the question, and its implications, see Bertolin, C., Périodes de 1-motifs et transcendance. J. Number Theory 97 (2002), no. 2, 204-221.

M.15. (p. 46) (7.4) There are similar calculations in 
Ogus, A., Griffiths transversality in crystalline cohomology. Ann. of Math. (2) 108 (1978), no. 2, 395-419, MR 80d:14012 (§3),

Ran, Ziv Cycles on Fermat hypersurfaces. Compositio Math. 42 (1980/81), no. 1, 121-142,

and, in a more general setting,

Aoki, Noboru, A note on complete intersections of Fermat type. Comment. Math. Univ. St. Paul. 35 (1986), no. 2, 231-245.

M.16. (p. 55) (7.16) For an elementary proof that $\mathbb{Q}\left(\zeta_{d}, \tilde{\Gamma}(\mathbf{a})\right)$ is Galois over $\mathbb{Q}$, see

Das, Pinaki, Algebraic gamma monomials and double coverings of cyclotomic fields. Trans. Amer. Math. Soc. 352 (2000), no. 8, 3557-3594. 